UTIG-02-04

hep-th/0403076

\title{
The $\mathcal{N}=1^{*}$ Theories on $R^{1+2} \times S^{1}$ with Twisted Boundary Conditions
}

\author{
Seok Kim ${ }^{1}$, Ki-Myeong Lee ${ }^{2,3}$, Ho-Ung $\mathrm{Yee}^{2}$, and Piljin $\mathrm{Yi}^{2}$ \\ ${ }^{1}$ School of Physics, Seoul National University, Seoul 151-747, Korea \\ ${ }^{2}$ Korea Institute for Advanced Study, Seoul 130-722, Korea \\ ${ }^{3 \dagger}$ Physics Department, University of Texas at Austin, Texas 78712, USA \\ Email: calaf2@snu.ac.kr,klee@kias.re.kr,ho-ung.yee@kias.re.kr, piljin@kias.re.kr \\ $\dagger$ (until the end of May, 2004)
}

\begin{abstract}
We explore the $\mathcal{N}=1^{*}$ theories compactified on a circle with twisted boundary conditions. The gauge algebra of these theories are the so-called twisted affine Lie algebra. We propose the exact superpotentials by guessing the sum of all monopole-instanton contributions and also by requiring $S L(2, Z)$ modular properties. The latter is inherited from the $\mathcal{N}=4$ theory, which will be justified in the $\mathrm{M}$ theory setting. Interestingly all twisted theories possess full $S L(2, Z)$ invariance, even though none of them are simply-laced. We further notice that these superpotentials are associated with certain integrable models widely known as elliptic Calogero-Moser models. Finally, we argue that the glueball superpotential must be independent of the compactification radius, and thus of the twisting, and confirm this by expanding it in terms of glueball superfield in weak coupling expansion.
\end{abstract}




\section{Contents}

1. Introduction 2

2. Theory 6

2.1 Twisted Boundary Condition on $S^{1} \quad 8$

2.2 Wilson-loop Symmetry Breaking 10

3. Monopoles and Superpotential 12

3.1 Classical Solution 13

3.2 Superpotential: Dilute Instanton Gas 15

3.3 Superpotential: Summing Over Higher Instantons 17

4. $S L(2, Z)$ Symmetry 19

$4.1 \quad S L(2, Z)$ and Four Types of Orientifold Planes 19

4.2 Compactification and M-theory Realization 20

4.3 M Theory Realization of Twisted Theories 22

5. $S L(2, Z)$ Symmetries and Exact Superpotential 23

$5.1 S L(2, Z)$ on Charge Lattice and Modular Transformation 24

$5.2 A_{2 r}^{(2)}$ Case 28

$5.3 A_{2 r-1}^{(2)}$ Case 29

$5.4 D_{r+1}^{(2)}$ Case 30

$5.5 E_{6}^{(2)}$ and $D_{4}^{(3)}$ Cases 31

6. Glueball Superpotential 32

$6.1 \mathcal{W}^{V Y}$ and Universality 35

6.2 Glueball Superpotential of Untwisted $A D E$ Theories 37

$6.3 A_{r}^{(1)}$ vs. $A_{r}^{(2)} 38$

$6.4 A_{2}^{(1)}$ vs. $A_{2}^{(2)} 41$

$6.5 D_{r}^{(1)}$ vs. $D_{r}^{(2)} 43$

$6.6 E_{6}^{(2)}$ and $D_{4}^{(3)}$

7. Conclusion 45 


\section{Introduction}

The $\mathcal{N}=1^{*}$ theory is obtained by adding masses for all three adjoint chiral supermultiplets of the $\mathcal{N}=4$ supersymmetric theory. It is well known that the classical vacuum structure of the $\mathcal{N}=1^{*}$ theory is characterized by embedding of $S U(2)$ algebra to the gauge algebra [1]. Quantum mechanically, however, the phenomena of gaugino condensation and chiral symmetry breaking further complicate the physics of vacua. The Seiberg-Witten curve for the $\mathcal{N}=2^{*}$ theory, where only two out of three chiral multiplets of the $\mathcal{N}=4$ theory have nonzero equal mass, was found early on in the study of Seiberg-Witten theory [2, 3]. Interestingly, the singularities of these curves were found to determine the vacuum structure of the corresponding $\mathcal{N}=1^{*}$ theory. Furthermore, it has been shown that the quantum $\mathcal{N}=1^{*}$ theory with $S U(N)$ gauge group inherits the $S L(2, Z)$ symmetry of the $\mathcal{N}=4$ theory, whereby the massive vacua of the quantum theory are transformed to each other [3].

On the other hand, the Seiberg-Witten curves of pure $\mathcal{N}=2$ super Yang-Mills theories with any semi-simple Lie group have been found to have a deep connection to classical integrable models. The Seiberg-Witten curves for the $\mathcal{N}=2$ pure YangMills theory turn out to be the spectral curves of the Toda-models [3, 4, 5, 6, [7, 8] ${ }^{1}$. Furthermore, the Seiberg-Witten curves for $\mathcal{N}=2^{*}$ theories with semi-simple Lie algebra are related to the so-called twisted elliptic Calogero-Moser models [6, 9, 10, [1].

The study of supersymmetric theories compactified on a circle was initiated in Ref. [15]. For the $\mathcal{N}=1$ pure Yang-Mills theory which is periodic on the circle, the gauge fields in the Cartan part of the gauge algebra may be dualized to chiral fields, and the supersymmetric effective potential as a function of these chiral fields has been obtained and found to be the twisted affine Toda potential [16, 17, 18, 19]. For the case of the $\mathcal{N}=1^{*} S U(N)$ theory compactified on a circle with $S U(N)$ gauge group, the effective superpotential was argued to be the potential of the elliptic $S U(N)$ Calogero-Moser model, on which the $S L(2, Z)$ acts as a modular transformation [20]. For $\mathcal{N}=1^{*}$ theories with other simple gauge groups with periodic boundary condition, there is some work on the superpotential [21], but it has not been fully explored.

In this paper, we wish to study the structure of $\mathcal{N}=1^{*}$ theories with arbitrary semi-simple gauge group compactified on a circle, but with a twist. When one of spatial directions is compactified on a circle, one may impose twisted boundary con-

\footnotetext{
${ }^{1}$ The connection between $\mathcal{N}=2$ theories and integrable models can be also realized in string theory [12, 13,14$]$.
} 
dition instead of periodic one, without breaking supersymmetry. Twisted boundary condition here means ${ }^{2}$

$$
\Phi\left(x_{4}+2 \pi R\right)=\boldsymbol{\sigma}\left[\Phi\left(x_{4}\right)\right],
$$

where $\boldsymbol{\sigma}$ is an outer automorphism acting on the adjoint representation with the property $\boldsymbol{\sigma}^{L}=1$ for some integer $L$, and $x_{4}$ is the compactified direction of radius $R$. Throughout this paper, we will adopt the notation, $\mathcal{G}^{(L)}$, for the theory obtained by twisting the corresponding $\mathcal{N}=1^{*}$ theory based on Lie algebra $\mathcal{G}$. The twistable $\mathcal{G}$ 's are exhausted by $A_{r}, D_{r}$, and $E_{6}$. We wish to find exact superpotentials of these theories by extending the above intricate relationships found on the theories with periodic boundary condition. A preliminary consideration of this problem has appeared in Ref. [22].

A much simpler version of this problem would be to consider the limit of the pure $\mathcal{N}=1$ theories on $R^{1+2} \times S^{1}$. This is obtained from $\mathcal{N}=1^{*}$ by letting all chiral multiplets infinitely massive. With periodic boundary condition and arbitrary simple gauge group, the effective superpotential has been calculated and found to be exactly the potential of the Toda model associated with the $\mathcal{N}=2$ theory [16, 17, 18, 19]. This Toda potential has a nice physical interpretation as arising from a supersymmetric generalization of the Polyakov mechanism, namely as nonperturbative contributions from fundamental monopole instantons on Euclidean three dimensional space time.

One nice aspect of this simpler $\mathcal{N}=1$ theory compactified on a circle is that the superpotential truncates down to a sum over "unit" monopole-instanton contributions. The usual semiclassical computation with the dilute gas approximation suffices. When one introduces twisted boundary condition along the circle, the pure $\mathcal{N}=1$ theory can be treated easily by using the same approach as in Ref. [18, 19]. In weak coupling limit, one calculate the effective superpotential from the contributions from fundamental monopole instantons. Our result on the effective superpotential for the $\mathcal{N}=1$ theories for all semi-simple gauge groups with twisted boundary condition also are given by the potential of the Toda-type model with definite normalization. The Toda model is associated to the twisted affine-algebra.

The superpotential turns out to be holomorphic and independent of the compactification radius and so is valid in any radius or coupling. However, the boundary condition becomes irrelevant in the infinite radius limit. Thus, the physics of ground states, vacuum degeneracy and the gluino condensation, should be identical to that of the theories with periodic boundary condition. This turns out to be borne out with

\footnotetext{
${ }^{2}$ Stringy realization of such a twist has been considered in Ref. [23, 24].
} 
help of the three rather nontrivial identities satisfied by the roots of the untwisted and twisted Kac-Moody algebra.

With three massive chiral multiplets in the adjoint representation in the $\mathcal{N}=$ $1^{*}$ theory, however, this nice Toda-like feature is no longer exact. One must sum over all possible combinations of monopole instantons to obtain the exact answer. Approaching this problem in the usual dilute gas approximation is obviously illfated, and we must turn to other methods. We first guess that the summation of all monopole instantons of a given type can be written in terms of the Weierstrass elliptic function as in the untwisted $S U(N)$ case of Ref. [20]. We will use the consistency with small coupling limit to the Toda potential and Weyl symmetry to reach a preliminary form of the superpotential.

The most powerful condition that will lead us to the correct answer is the $S L(2, Z)$ symmetry. As was mentioned already, the $S L(2, Z)$ of $\mathcal{N}=4 S U(N)$ theories is known to be inherited by their $\mathcal{N}=1^{*}$ cousins. We will assume that this inheritance is generic, and proceed by determining the $S L(2, Z)$ modular properties of the $\mathcal{N}=4$ theories with twisted boundary condition. Then the problem is reduced to picking out appropriate superpotential which is modular under $S L(2, Z)$ transformations.

The middle part of this paper will discuss in much detail the issue of the $S L(2, Z)$ in $\mathcal{N}=4$ theories with twisted boundary condition, where we will make a crucial use of M-theoretical and stringy realizations of the twisted $\mathcal{N}=4$ theories. At the end of this discussion, we will find that all "exact" superpotentials correspond to the potentials of elliptic Calogero-Moser models, once again demonstrating the close connection between Seiberg-Witten theories, compactified $\mathcal{N}=1$ theories, and integrable models.

Recall that the integrable models related to $\mathcal{N}=2^{*}$ theories have been found to be the elliptic Calogero-Moser models [6, 10], or rather, to be more precise, the corresponding integrable models are determined by the co-root vectors of the gauge group, leading to the twisted Calogero-Moser models [11. Thus, given the usual relationship between $\mathcal{N}=2$ Seiberg-Witten curve and $\mathcal{N}=1$ superpotential, the effective superpotentials for $\mathcal{N}=1^{*}$ theories with periodic boundary condition are expected to be related to the integrable models of twisted elliptic Calogero-Moser models [11.

In contrast, for the theory with twisted boundary condition, we find that the effective superpotentials are related to the untwisted elliptic Calogero-Moser models, $C_{r}, B_{r}, F_{4}$, and $G_{2}$ types, respectively, for the group $A_{2 r-1}^{(2)}, D_{r+1}^{(2)}, E_{6}^{(2)}$, and $D_{4}^{(3)}$, 
and a twisted elliptic Calogero-Moser model of $B C_{r}$ type for the group $A_{2 r}^{(2)}$. These integrable models have been studied in Ref. 11, 25, 26.

Our computation, which covers both twisted and untwisted cases, is actually more specific than those in the literature, as we have unambiguous normalization factors for every term in the superpotential, including one which only depends on the coupling constant. We fixed them utilizing small coupling limit, Weyl symmetry, and modular properties under $S L(2, Z)$. This allows us the detailed exploration of the full modular property of the effective potential. We would like to take this as an independent evidence for the validity of our approach.

We subject our effective potential to another nontrivial consistency check. The effective superpotential turns out to be independent of the compactification radius. While this does not immediately imply that the physics is independent of boundary condition, we can deduce that the physics of gaugino condensation and vacua labelled by this order parameter are independent of the boundary condition. This independence may be rather surprising since the shape of superpotential does depend on whether the theory is twisted or not.

For this, we introduce the glueball superfield, $S \sim \operatorname{Tr} W^{\alpha} W_{\alpha}$, a la Veneziano and Yankielowicz [27], and extract the glueball superpotential. This is achieved by a 'dual' or 'mirror' transformation in weak coupling series, which is a kind of Legendre transformation [28]. The leading term of the superpotential, which is the Toda potential of the $\mathcal{N}=1$ theory, is mapped to the Veneziano-Yankielowicz potential for the glueball superfield after this dual transformation. Higher order monopole instanton corrections of the $\mathcal{N}=1^{*}$ theory are mapped to higher order terms in the glueball superpotential. ${ }^{3}$ We find the glueball superpotential in a series, by first doing weak coupling expansion of our superpotential and then taking the dual or mirror transformation. We find that the glueball superpotentials obtained from our effective superpotentials for periodic and twisted boundary conditions are identical to each other in a few leading orders.

The plan of this paper is as follows. In Sec.2, we introduce the $\mathcal{N}=1^{*}$ theory on $R^{1+2} \times S^{1}$ with periodic and twisted boundary conditions. We focus here on the possible large gauge transformations and the range of the Wilson-loop variables. In Sec.3, we study the monopole instantons, or fractional instantons, classically and

\footnotetext{
${ }^{3}$ The $\mathcal{N}=1^{*}$ theory with simply laced groups has been studied in series to fourth order in the glueball superfield [29]. Their method is the matrix model calculation developed by Dijkgraaf and Vafa [30]. This glueball superpotential has been checked for the $\mathcal{N}=1^{*}$ theory with the $A_{r}=S U(r+1)$ gauge theory. However we find a small discrepancy with the result in Ref.[29] at the fourth order for other simply laced groups, like $D_{r}, E_{6}, E_{7}, E_{8}$.
} 
quantum mechanically, which leads to the preliminary version of the superpotential. In Sec.4, we present the M-theory picture of the $\mathcal{N}=4$ theory with various classical groups and boundary conditions, to see that all twisted cases are self-dual under $S L(2, Z)$. In Sec.5, we impose the $S L(2, Z)$ modular property for the twisted case, which fixes the superpotential for a given group uniquely. In Sec.6, we obtain the glueball superpotentials in weak coupling series by the dual transformation and show that they are independent of the boundary condition. In Sec. 7, we conclude with some remarks. In the appendix, we also record glueball superpotentials for (untwisted) theories associated with non-simply-laced algebra, as well as provide various mathematical facts on Lie Algebra, affine Lie algebra, and twisted affine Lie algebra.

\section{Theory}

The theories we consider are $\mathcal{N}=1$ and $\mathcal{N}=1^{*}$ supersymmetric Yang-Mills theory with a simple Lie algebra $\mathcal{G}$ of rank $r$. The gauge field is defined as $A_{\mu}=A_{\mu}^{i} T^{i}$ with the orthonormal elements $T^{i}$ of Lie algebra satisfying the inner products $\operatorname{Tr} T^{i} T^{j}=$ $\delta^{i j}$ as given in Appendix B. We choose the Euclidean action for the gauge field to be

$$
S_{E}=\frac{1}{2 e^{2}} \int d^{4} x \operatorname{Tr}\left(F_{\mu \nu} F^{\mu \nu}\right)-\frac{i \theta}{16 \pi^{2}} \int d^{4} x \operatorname{Tr}\left(F_{\mu \nu} \tilde{F}^{\mu \nu}\right) .
$$

The $\mathcal{N}=1$ supersymmetric Yang-Mills theory has an additional term for the gaugino field $\lambda$. With the holomorphic coupling constant

$$
\tau=\frac{\theta}{2 \pi}+\frac{4 \pi i}{e^{2}}
$$

and the glueball superfield of the chiral gauge field strength $W_{\alpha}$,

$$
S=\frac{1}{16 \pi^{2}} \operatorname{Tr}\left(W_{\alpha} W^{\alpha}\right)
$$

the Minkowski Lagrangian for the $\mathcal{N}=1$ supersymmetric theory is

$$
L=\left.2 \pi i \tau S\right|_{\theta^{2}}+\text { h.c. }
$$

The $\mathcal{N}=1^{*}$ theory has three more chiral fields $\Phi_{i}, i=1,2,3$ in the adjoint representation with the standard kinetic term and an additional $F$ term,

$$
W=\operatorname{Tr}\left(\left(\Phi_{1},\left[\Phi_{2}, \Phi_{3}\right]\right)+\sum_{i=1}^{3} m_{i} \Phi_{i}^{2}\right) .
$$

When all $m_{i}$ vanish, the theory becomes the maximally supersymmetric $\mathcal{N}=4$ theory. When one of them vanishes and the rest two become identical, the theory becomes the $\mathcal{N}=2^{*}$ theory. 
Classically, the vacuum of the $\mathcal{N}=1$ theory is trivial and unique. The $\mathcal{N}=1^{*}$ theory has degenerate classical vacua,

$$
<\Phi_{i}>=\sqrt{\frac{m_{1} m_{2} m_{3}}{m_{i}}} J_{i},
$$

where $J_{i}$ 's are the $S U(2)$ generators embedded in the Lie algebra $\mathcal{G}$ [1].

Quantum mechanically, physics is much richer. Perturbatively the $\mathcal{N}=1$ theory is asymptotically free in high energy with a confinement scale $\Lambda$. The vacuum of the $\mathcal{N}=1$ theory is strongly interacting and confining with a gaugino condensation. There exist degenerate supersymmetric vacua. The vacuum physics can be elegantly summarized by the Veneziano-Yankielowicz potential [27] for the glueball field $S$, which is a holomorphic function of the chiral coupling constant $\tau$ and the chiral glueball superfield $S$ such that

$$
\mathcal{W}_{G}^{V Y}=h S-S \ln \left(\frac{S}{\Lambda^{3}}\right)^{h},
$$

where

$$
\Lambda^{3}=m^{3} e^{2 \pi i \tau(m) /(3 h)} \prod_{a=0}^{r}\left[\frac{k_{a}^{*} \boldsymbol{\alpha}_{a}^{2}}{2}\right]^{k_{a}^{*}},
$$

with the simple roots $\boldsymbol{\alpha}_{a}$ of the extended Dynkin diagram for the gauge Lie algebra and the comarks $k_{a}^{*}$ which is discussed in Appendix B. The parameter $\Lambda$ is the confinement energy scale found explicitly in Ref. [19] and the dual Coxeter number $h$ is defined as the sum of comarks $h=\sum_{a=0}^{r} k_{a}^{*}$.

Here we consider the $\mathcal{N}=1$ theory in the $\mathcal{N}=1^{*}$ context which is ultra-violet finite and the coupling $\tau(m)$ is defined on the mass scale $m=m_{1}=m_{2}=m_{3}$. A vacuum is one of the stationary points of this superpotential. The number of degenerate vacua is $h$ and each vacuum is characterized by an integer $k$ such that $0 \leq l \leq h-1$. The glueball expectation value at a vacuum is then

$$
<S>=\frac{1}{16 \pi^{2}}<\operatorname{Tr} \lambda \lambda>=\Lambda^{3} e^{\frac{2 \pi i l}{h}}, \quad l=0,1, \ldots h-1,
$$

where $\mathcal{W}_{V Y}$ is stationary.

The $\mathcal{N}=4$ theory with the $S U(N)$ gauge group is finite perturbatively, and has the nontrivial strong-weak or $S L(2, Z)$ modular symmetry under which the coupling constant transforms as

$$
\tau \rightarrow \frac{a \tau+b}{c \tau+d},
$$

for integers $a, b, c, d$ such that $a d-b c=1$. The $\mathcal{N}=1^{*}$ theory is finite at energy scale larger than mass scales $m_{i}$. For the semiclassical calculation, we are initially 
interested in the weak coupling limit where $e^{2} / 4 \pi^{2}$ is small at high energy. The low energy physics of the $\mathcal{N}=1^{*}$ theory would depend on which classical vacuum one resides. If there is an unbroken nonabelian subgroup, its coupling starts to grow and its matters become confining at the energy scale much smaller than $m_{i}$.

The classical vacuum (2.6) of the $\mathcal{N}=1^{*}$ theory has a rich quantum structure as any unbroken nonabelian subgroup runs to the confining phase, inducing also gluino condensation and degenerate vacua. When the embedding is maximal, the gauge symmetry is completely broken at the mass scale $m$ and all particles become massive. When the embedding is trivial, the gauge symmetry is not broken at all and so one ends up with the confining phase. When the embedding is nontrivial but not maximal, the low energy physics can depend on symmetry breaking pattern. If the $S U(2)$ embedding has only irreducible pieces of identical dimension, only unbroken symmetry is a nonabelian subgroup and so the theory reduces to the confining phase of smaller nonabelian subgroup. If the embedding has irreducible pieces of different dimensions, there would be an unbroken abelian symmetry whose photon remains massless in the infrared. For the theory with $S U(N)$ gauge group, all massive vacua, with no massless modes, transform each other under the $S L(2, Z)$ transformation, regardless whether they are in Higgs phase or confining phase[3].

\subsection{Twisted Boundary Condition on $S^{1}$}

We compactify one of the spatial directions along a circle of radius $R$, so that the fourth coordinate is cyclic,

$$
x^{4} \sim x^{4}+2 \pi R .
$$

One could impose periodic boundary condition on the gauge field,

$$
A_{\mu}\left(x^{0}, x^{1}, x^{2}, x^{4}+2 \pi R\right)=A_{\mu}\left(x^{0}, x^{1}, x^{2}, x^{4}\right)
$$

as well as other fields, which does not violate supersymmetry. Thus we can use the holomorphic properties of the effective superpotential in the compactified theories.

For a Lie algebra $\mathcal{G}$ whose Dynkin diagram has a symmetry, there exists an outer automorphism $\boldsymbol{\sigma}$ on the Lie algebra as argued in the Appendix D such that $\boldsymbol{\sigma}^{L}=1$. We choose twisted boundary condition on all fields so that the gauge field satisfies

$$
A_{\mu}\left(x^{0}, x^{1}, x^{2}, x^{4}+2 \pi R\right)=\boldsymbol{\sigma}\left(A_{\mu}\left(x^{0}, x^{1}, x^{2}, x^{4}\right)\right),
$$

with the same boundary condition on all other fields. This twisted boundary condition also preserves supersymmetry. 
For periodic boundary condition, we may take $L=1$. When there is a nontrivial outer automorphism for Lie algebra $\mathcal{G}$ so that $L \neq 1$, the elements of the Lie algebra $\mathcal{G}$, as shown in Appendix D, can be classified to the sets, $\mathcal{G}_{n}$ 's, of the eigenstates of the automorphism as $\boldsymbol{\sigma}\left(\mathcal{G}_{n}\right)=e^{2 \pi i n / L} \mathcal{G}_{n}$, where $n=0,1, \ldots L-1$. Since $\left[\mathcal{G}_{m}, \mathcal{G}_{l}\right]=$ $\mathcal{G}_{m+l \bmod L}$, only $\mathcal{G}_{0}$ is a Lie algebra and other sets would belong to representations of $\mathcal{G}_{0}$. For the Lie algebra $A_{r}, D_{r}, E_{6}$, an outer automorphism with $L=2$ is allowed. For $D_{4}$, one can also have an outer automorphism of $L=3$.

The gauge fields in the Lie algebra $\mathcal{G}$ can be expressed in terms of $\mathcal{G}_{n}$ basis. Those that are associated with the generators $\mathcal{G}_{n}$ can be Fourier-expanded as

$$
A_{\mu}^{i}\left(x^{4}\right) T^{i}=\sum_{m=-\infty}^{\infty} \sum_{n=0}^{L-1} A_{\mu}^{i,(m, n)} e^{-\frac{i x^{4}}{R}\left(m+\frac{n}{L}\right)} T^{i}=\sum_{m=-\infty}^{\infty} \sum_{n=0}^{L-1} A_{\mu}^{i,(m, n)} T_{m+\frac{n}{L}}^{i}
$$

defining

$$
T_{m+\frac{n}{L}}^{i} \equiv e^{-\frac{i x^{4}}{R}\left(m+\frac{n}{L}\right)} T^{i}, \text { when } T^{i} \in \mathcal{G}_{n} .
$$

Then the set $\left\{T_{m+\frac{n}{L}}^{i}\right\}$ of these generators form a twisted affine Lie algebra $\mathcal{G}^{(L)}$.

The gauge fields without the $x^{4}$ dependent component would have no KaluzaKlein mass term. Thus with twisted boundary condition, the gauge algebra $\mathcal{G}$ of rank $r$ in the three dimensional limit is reduced to a smaller simple Lie algebra $\mathcal{G}_{0}$ of rank $r^{\prime}<r$. The Cartan subalgebra of $\mathcal{G}_{0}$ is of dimension $r^{\prime}$. Since $\left[\mathcal{G}_{0}, \mathcal{G}_{n}\right] \in \mathcal{G}_{n}$, $\mathcal{G}_{n}$ belongs to a representation of $\mathcal{G}_{0}$ and every element of the $\mathcal{G}_{n}$ defines a weight of the Lie algebra $\mathcal{G}_{0}$. The simple roots $\boldsymbol{\beta}_{a}, a=1,2, \ldots r^{\prime}$ of $\mathcal{G}_{0}$ and the lowest negative weight $\boldsymbol{\beta}_{0}$ of $\mathcal{G}_{1}$ define the Dynkin diagram of the twisted affine Lie algebra $\mathcal{G}^{(L)}$, which is shown in Figure 3 in Appendix E.

In the twisted affine algebra $\mathcal{G}^{(L)}$, each root vector $\boldsymbol{\beta}$ of $\mathcal{G}_{0}$ is extended to a $\operatorname{root}(\boldsymbol{\beta}, m)$ with an integer $m$ with the corresponding step operator $E_{\boldsymbol{\beta}}^{m}$, and each weight vector $\boldsymbol{\beta}$ of $\mathcal{G}_{n}$ is extended to a $\operatorname{root}\left(\boldsymbol{\beta}, m+\frac{n}{L}\right)$ with the corresponding step operator $E_{\boldsymbol{\beta}}^{m+\frac{n}{L}}$. Without the central term in the twisted affine algebra like the case studied here, the square size of these root is just given by $\boldsymbol{\beta}^{2}$ regardless of the degree $d=m+n / L$.

For the gauge theory with twisted boundary condition, the allowed gauge transformation $g\left(x^{i}, x^{4}\right)$ should preserve the twisted affine Lie algebra relations. There are small gauge transformations which are generated by $\mathcal{G}_{0}$ and independent of $x^{4}$. There are also large gauge transformations with $x^{4}$ dependence. They would play a crucial role in determining the classical vacuum structure of the theory as we will see. 


\subsection{Wilson-loop Symmetry Breaking}

We focus on the Coulomb phase where classically the vacuum expectation value of the scalar fields $\Phi_{i}$ vanishes. On a circle, the fourth component gauge field can take a constant nonvanishing expectation value in the Cartan subalgebra,

$$
\left\langle A_{4}\right\rangle=\boldsymbol{\xi} \cdot \boldsymbol{H}
$$

with $\boldsymbol{\sigma}(\boldsymbol{\xi})=\boldsymbol{\xi}$ and so it belongs to $\mathcal{G}_{0}$. This configuration has zero field strength and so is a classical vacuum configuration. For the generic expectation value of $\boldsymbol{\xi}$, the gauge symmetry would be broken to its maximal abelian subgroup $U(1)^{r^{\prime}}$. The rest of the gauge components would get the mass terms due to the Higgs mechanism and the Kaluza-Klein mass.

When the gauge symmetry is maximally broken to its abelian subgroup, we introduce a dimensionless scalar field $\varphi$ of $r^{\prime}$ components such that

$$
\boldsymbol{\varphi} \cdot \boldsymbol{H}=2 \pi R A_{4}
$$

and so $\langle\boldsymbol{\varphi}\rangle=2 \pi R \boldsymbol{\xi}$. The Wilson loop expectation value in the classical vacuum would be $\left\langle P \exp \left(i \int_{0}^{2 \pi R} d x^{4} A_{4}\right)\right\rangle=\exp (i \varphi \cdot \boldsymbol{H})$.

The allowed large gauge transformations are those which leave the twisted affine algebra relations invariant. Easier ones are those that transform even degree generators to even degree ones and odd degree ones to odd degree ones. On the other hand, it is possible to have large gauge transformations which would transform even degree generators to odd ones and vice versa. Let us try the general form of an allowed large gauge transformation as

$$
U=e^{\frac{-i x^{4}}{R} \boldsymbol{w}^{*} \cdot \boldsymbol{H}}
$$

where $\boldsymbol{w}^{*}=\sum_{a=1}^{r^{\prime}} c_{a} \boldsymbol{w}_{a}^{*}$ with $\boldsymbol{w}_{a}^{*}$ being the fundamental coweight vector so that $\boldsymbol{w}_{a}^{*} \cdot \boldsymbol{\beta}_{b}=\delta_{a b}$. Constraints on the coefficient $c_{i}$ arise as we require that the gauge transformation of any step operator

$$
U E_{m+\frac{n}{L}}^{\boldsymbol{\beta}} U^{\dagger}=e^{-\frac{i x^{4}}{R} \boldsymbol{w}^{*} \cdot \beta} E_{m+\frac{n}{L}}^{\boldsymbol{\beta}},
$$

should be a step operator with $\operatorname{root}\left(\beta, m+\frac{n}{L}+\boldsymbol{w}^{*} \cdot \boldsymbol{\beta}\right)$. This is easy to work out for all twisted affine algebra by studying each case. For $A_{2 r}^{(2)}$ the allowed ones are given by the integer lattice defined by the set of one half $\boldsymbol{w}_{a}^{*} / 2$ of each fundamental co-weight. For the rest of the affine algebra the allowed ones are given by the weight lattice $\Lambda_{w}$ generated by the fundamental weights $\boldsymbol{w}_{a}$ not by coweights $\boldsymbol{w}_{a}^{*}$, contrasted to the theories with non simply-laced untwisted affine Lie algebra [19]. 
These allowed large gauge transformations can be used to put the vacuum expectation value to lie in a fundamental cell. For the theory with $A_{2 r^{\prime}}^{(2)}$ the fundamental cell is

$$
0 \leq\langle\boldsymbol{\varphi}\rangle \cdot \boldsymbol{\beta}_{a}<\pi, a=1, \ldots r^{\prime} .
$$

For the theories with the rest of twisted affine algebra, the fundamental cell is

$$
0 \leq\langle\boldsymbol{\varphi}\rangle \cdot \boldsymbol{\beta}_{a}^{*}<2 \pi, \quad a=1, \ldots r^{\prime}
$$

where $\boldsymbol{\beta}_{a}$ is the simple roots of $\mathcal{G}_{0}$. This periodicity of the vacuum expectation value will be manifest in the supersymmeric effective potential obtained later. For the reference, we enlist the vacuum expectation value for the theory with periodic boundary condition also,

$$
0 \leq\langle\boldsymbol{\varphi}\rangle \cdot \boldsymbol{\alpha}_{a}<2 \pi, \quad a=1, \ldots r,
$$

where $\boldsymbol{\alpha}_{a}$ are the simple roots of $\mathcal{G}$. This is again consistent with the effective superpotential.

When nontrivial Wilson-loop breaks the gauge symmetry $\mathcal{G}_{0}$ to abelian subgroup $U(1)^{r^{\prime}}$, besides large gauge transformation, there are still discrete nonabelian gauge transformations, which are Weyl-reflections on the root vectors of $\mathcal{G}_{0}$. This reduces the range of the vacuum expectation value. Especially, one can always find Weyl reflections to reduce the range of the expectation value in the fundamental cell to

$$
\frac{4 \pi}{L \boldsymbol{\beta}_{0}^{2}}+\langle\boldsymbol{\varphi}\rangle \cdot \boldsymbol{\beta}_{0}^{*} \geq 0
$$

This condition reduces the fundamental cell to a smaller subset, which we may call the fundamental alcove. This reduced vacuum moduli space can be characterized by a quotient space,

$$
\varphi \in \mathcal{M}_{\text {vacuum }}=\frac{R^{r^{\prime}}}{2 \pi \cdot \Lambda_{L G} \rtimes W_{\mathcal{G}_{0}}},
$$

where $\Lambda_{L G}$ is the lattice of the allowed large gauge transformations made by $\boldsymbol{w}^{*}$, and $W_{\mathcal{G}_{0}}$ is the Weyl group on $\mathcal{G}_{0}$.

The low energy dynamics of maximally broken theory would be $x^{4}$-independent and purely abelian with the Euclidean action,

$$
S_{1}=\frac{2 \pi R}{e^{2}} \int d^{3} x\left(\frac{1}{4 \pi^{2} R^{2}}\left(\partial_{i} \boldsymbol{\varphi}\right)^{2}+\mathbf{F}_{i j}^{2}\right)-\frac{2 \pi i \theta R}{8 \pi^{2}} \int d^{3} x \epsilon_{i j k} \partial_{i} \boldsymbol{\varphi} \cdot \mathbf{F}_{j k},
$$

where $\mathbf{F}_{m n}$ is the purely abelian three dimensional gauge field strength. One can dualize the three dimensional abelian gauge field by introducing a dimensionless 
scalar field $\boldsymbol{\sigma}$, adding a topological action

$$
S_{2}=-\frac{i}{4 \pi} \int d^{3} x \epsilon_{i j k} \partial_{i} \boldsymbol{\sigma} \cdot \mathbf{F}_{j k}
$$

and regarding $\boldsymbol{\sigma}$ and $\mathbf{F}_{m n}$ as dynamical variables. Integrating over $\boldsymbol{\sigma}$ imposes that $\mathbf{F}_{m n}$ should be a gauge field strength. Integrating over $\mathbf{F}_{m n}$ and introducing a complex scalar field

$$
\boldsymbol{z}=i(\tau \boldsymbol{\varphi}+\boldsymbol{\sigma})
$$

lead to a simplification of the total action $S_{1}+S_{2}$ to the action

$$
S_{\text {classical }}=\frac{1}{8 \pi^{2} R} \int d^{3} x \frac{1}{\operatorname{Im} \tau} \partial_{i} \boldsymbol{z}^{\dagger} \partial_{i} \boldsymbol{z}
$$

Including a gaugino field leads to a chiral superfield $\boldsymbol{X}$ in four dimensional sense whose bosonic field is the complex scalar field $\boldsymbol{z}$. We consider the case where the compactification radius $R$ is much smaller than the confinement scale. In other words we consider the case where $\Lambda \ll R^{-1} \ll m$. The initial coupling constant $e^{2}(m)$ is small and so is $e^{2}\left(R^{-1}\right)$. Thus we are calculating in the weak coupling limit. After integrating out the massive modes of mass scale $1 / R$ or more, we end up with a low energy effective action

$$
S_{\text {eff }}=2 \pi R \int d^{3} x\left(\left.\mathcal{K}\left(X, X^{\dagger}\right)\right|_{\theta^{2} \bar{\theta}^{2}}+\left.\mathcal{W}(X)\right|_{\theta^{2}}+\left.\mathcal{W}\left(X^{\dagger}\right)\right|_{\bar{\theta}^{2}}\right)
$$

in leading order in derivative expansion. The holomorphic quantities in the theory are the chiral fields $\boldsymbol{X}$, the coupling $\tau(m)$ defined at the scale $m$ and the mass parameter $m$. (Here we put all the mass scales of the $\mathcal{N}=1^{*}$ theory to be equal, $m_{i}=m$, for simplicity.) The holomorphic part of the effective action would depend only on these chiral quantities,

$$
\mathcal{W}_{\text {eff }}=\mathcal{W}(\boldsymbol{X}, m, \tau)
$$

\section{Monopoles and Superpotential}

We are interested in the effective action near the confining vacuum where $\left.<\Phi_{i}\right\rangle=0$, and so all $\Phi_{i}$ supermultiplets have mass $m$. In the low energy effective action we are looking for, there is no perturbative correction to the $F$-term or the holomorphic superpotential. Thus, we are interested in nonperturbative contributions to the effective superpotential. This effective superpotential has been found for the $\mathcal{N}=1$ theory with $S U(N)$ gauge group [16, 18] and other simple gauge groups [17, 19] and 
also for the $\mathcal{N}=1^{*}$ theory with $S U(N)$ gauge group and periodic boundary condition [20]. The nonperturbative effects are due to magnetic monopole instantons, which can be regarded as fractional instantons. Such configurations are possible as the classical vacuum of the abelian gauge theory has degeneracy due to the magnetic flux.

The natural nonperturbative contributions come from instanton configurations in four dimensional Euclidean spacetime $R^{3} \times S^{1}$, which satisfy self-dual equations

$$
B_{i} \equiv \frac{1}{2} \epsilon_{i j k} F_{j k}=F_{i 4}
$$

where $i=0,1,2$. As one spatial direction $x^{4}$ is compactified to a circle, we can have Wilson-loop gauge symmetry breaking. Let us assume a maximal symmetry breaking to the abelian subgroups. In this case it turns out that the solutions of the self-dual equations are made of more than just instantons. There are magnetic monopoles which can be regarded as fractional instantons.

\subsection{Classical Solution}

These magnetic monopoles and instanton solutions have been extensively studied before. Initially W. Nahm studied calorons in the context of ADHM contruction for instantons and Nahm's equation for magnetic monopoles [31]. When there is no Wilson-loop symmetry breaking, there is a single caloron or instanton solution found by periodic array. In the string theory context, the relation between instantons and magnetic monopole are realized as D0 branes on D4 branes wrapping on $R^{3} \times S^{1}$ are T-dualized to D3 brane on the dual circle warpped by D1 branes, where some class of solutions are found with Wilson loop symmetry breaking [32. Explicit solution for a single instanton in the $\mathrm{SU}(2)$ gauge theory has been found when the gauge symmetry is broken to $U(1)$ by the Wilson loop symmetry breaking [33]. In Ref. [34], the ADHM contruction was T-dualized to the Nahm construction with nontrivial Wilson loop symmetry breaking and caloron solutions are explored. For the theory with periodic boundary condition and any simple gauge group of rank $r$ which is maximally broken by the Wilson loop, a single instanton is made of $r+1$ constituent fundamental monopoles 35. For the theories with twisted boundary condition, some aspects of Wilson loop symmetry breaking and instantons are studied [22].

Here we provide enough detail for the sake of completeness. Let us first write down a single magnetic monopole solution in the $S U(2)$ gauge theory. With the asymptotic value of the Higgs field $u$, the BPS solution is given as

$$
V_{i}^{a}(\mathbf{r}, u)=\epsilon_{a i j} \hat{r}^{j}\left(\frac{1}{r}-\frac{u}{\sinh u r}\right), \quad \Phi^{a}(\mathbf{r}, u)=\hat{r}^{a}\left(\frac{1}{r}-u \operatorname{coth} u r\right),
$$


which satisfies the BPS equation $\partial_{i} \Phi^{a}+\epsilon^{a b c} V_{i}^{b} \Phi^{c}=\epsilon_{i j k}\left(\partial_{j} V_{k}^{a}+\epsilon^{a b c} V_{j}^{b} V_{k}^{c} / 2\right)$.

For our theory with twisted boundary condition, we can choose any root $(\boldsymbol{\beta}, m+$ $\frac{n}{L}$ ) of degree $m+n / L$ of the twisted affine algebra $\mathcal{G}^{(L)}$ and its corresponding step operator $E_{\boldsymbol{\beta}}^{m+n / L}$. There are corresponding $S U(2)$ generators,

$$
t^{1}=\frac{1}{2}\left(E_{\boldsymbol{\beta}}^{m+n / L}+E_{-\boldsymbol{\beta}}^{-m-n / L}\right), \quad t^{2}=\frac{1}{2 i}\left(E_{\boldsymbol{\beta}}^{m+n / L}-E_{-\boldsymbol{\beta}}^{-m-n / L}\right), t^{3}=\frac{1}{2} \boldsymbol{\beta}^{*} \cdot \boldsymbol{H} .
$$

Note that the exchange of two $\operatorname{roots}(\boldsymbol{\beta}, m+s)$ and $(-\boldsymbol{\beta},-m-s)$ in the above definition is just a $\pi$-rotation along $t^{3}$ direction. We can embed the monopole solution (3.2) with these $\mathrm{SU}(2)$ generators. First we note that

$$
t^{a}\left(x^{4}\right)=e^{-i(m+n / L) x^{4} t^{3} / R} \tilde{t}^{a} e^{+i(m+n / L) x^{4} t^{3} / R},
$$

for $x^{4}$-independent $\tilde{t}^{a}$. Here the solution of the self-dual equation $B_{i}=F_{i 4}$ is then

$$
A_{i}=V_{i}^{a}(\mathbf{r}, u) t^{a}, \quad A_{4}=\left(\boldsymbol{\xi}-\frac{1}{2}(\boldsymbol{\xi} \cdot \boldsymbol{\beta}) \boldsymbol{\beta}^{*}\right) \cdot \boldsymbol{H}+\Phi^{a}(\mathbf{r}, u) t^{a},
$$

where

$$
u=\boldsymbol{\xi} \cdot \boldsymbol{\beta}+\frac{1}{R}\left(m+\frac{n}{L}\right) .
$$

The Euclidean action for the magnetic monopole is then

$$
S_{E}=\frac{4 \pi}{e^{2}} \frac{2}{\boldsymbol{\beta}^{2}} 2 \pi R\left|\boldsymbol{\xi} \cdot \boldsymbol{\beta}+\frac{1}{R}\left(m+\frac{n}{L}\right)\right| .
$$

(However $e^{-i(m+n / L) x^{4} t^{3} / R}$ is not in general an allowed large gauge transformation and so the monopole would be intrinsically $x^{4}$ dependent [35, 22].)

The detailed zero mode analysis of these monopole solutions would lead to the constituent monopole structure. Those with only four zero modes, three for its position and one for the phase, would be called fundamental monopoles. The rest of the monopole configurations would be composite of these fundamental monopoles. In this analysis 35, 22, it is crucial to transform the $A_{4}$ expectation values by large gauge transformations and by Weyl reflections so that it lies in the fundamental alcove defined by Eqs. (2.20,2.21,2.23).

The solutions with only four zero modes are those for the simple roots $\left(\boldsymbol{\beta}_{a}, 0\right), a=$ $1, . . r^{\prime}$ and the lowest root $\left(\boldsymbol{\beta}_{0}, 1\right)$. (Of course the solutions for negative of the above roots appearing in the Dynkin diagram is just a gauge transformation of the original one.) The Euclidean action for each fundamental monopole becomes

$$
S_{0}=\frac{8 \pi^{2}}{e^{2}}\left(\frac{2}{L \boldsymbol{\beta}_{0}^{2}}+\frac{1}{2 \pi}\langle\boldsymbol{\varphi}\rangle \cdot \boldsymbol{\beta}_{0}^{*}\right), S_{a}=\frac{8 \pi^{2}}{e^{2}} \frac{1}{2 \pi}\langle\boldsymbol{\varphi}\rangle \cdot \boldsymbol{\beta}_{a}^{*}, a=1,2, \ldots r^{\prime} .
$$


In the fundamental alcove we consider, the above actions are all positive. Let us consider a configuration which is made of $\tilde{k}_{a}^{*}, a=0,1, \ldots r^{\prime}$ number of $\boldsymbol{\beta}_{a}$ fundamental monopoles where $\tilde{k}_{a}^{*}$ are the comarks of the twisted affine algebra $\hat{\mathcal{G}}^{(L)}$ considered in Appendices B and E and shown explicitly at Figure 3 of Appendix E. We assume that it is possible to superpose self-dual magnetic monopole configurations to obtain another self-dual configurations, which seems reasonable. As the comarks $\tilde{k}_{a}^{*}$ satisfy the following relations,

$$
\sum_{a=0}^{r^{\prime}} \tilde{k}_{a}^{*} \boldsymbol{\beta}_{a}^{*}=0, \quad h=\sum_{a=0}^{r^{\prime}} \tilde{k}_{a}^{*}, \quad \frac{2 \tilde{k}_{0}^{*}}{L \boldsymbol{\beta}_{0}^{2}}=1,
$$

with the dual Coxeter number $h$, the above composite configuration would have $4 h$ zero modes, zero magnetic charge and the action of value $8 \pi^{2} / e^{2}$. This is exactly the property of a single instanton configuration on $R^{3} \times S^{1}$. Thus one can regard instantons as being made of constituent magnetic monopoles, which carry fractional instanton charges given by their action.

Any general self-dual configurations would be characterized by a $r^{\prime}+1$ nonnegative integer set $\left\{n_{a}, a=0,1, \ldots r^{\prime}\right\}$, which count the number $n_{a}$ of the fundamental monopole $\boldsymbol{\beta}_{a}^{*}$. The total magnetic charge $\mathbf{g}$ and action $S$ of such configuration would be

$$
\mathbf{g}=\sum_{a=0}^{r^{\prime}} n_{a} \boldsymbol{\beta}_{a}^{*}, \quad S=\sum_{a=0}^{r^{\prime}} n_{a} S_{a}
$$

where $S_{a}, a=0,1, \ldots r^{\prime}$ are those in Eq. (3.8). The number of the zero modes would be $4 \sum_{a=0}^{r^{\prime}} n_{a}$.

\subsection{Superpotential: Dilute Instanton Gas}

Let us consider the quantum effect of the magnetic monopole instantons on $R^{1+2} \times S^{1}$. Classically a theory with $U(1)^{r^{\prime}}$ gauge group has degenerate vacua whose magnetic flux can take arbitrary value. The magnetic monopole instantons tunnel between these degenerate vacua. When we consider the long distance physics whose length scale is much larger than the compactification radius $R$, or the magnetic monopole size scale, the magnetic monopole instantons induce an effective action.

To find this effective action, we reconsider the action (2.26) for the dual photon by regarding $\mathbf{F}_{j k}$ as a field strength and write the action as a boundary value,

$$
S_{2}=-\frac{i}{2 \pi} \int_{S^{2}} d x^{m} \boldsymbol{\sigma} \cdot \mathbf{B}_{m}
$$

where $\mathbf{B}_{i}=\epsilon_{i j k} \mathbf{F}_{j k} / 2$. For a magnetic monopole of magnetic charge $\mathbf{g}$, asymptotically $\mathbf{B}_{m}=(\mathbf{g} \cdot \boldsymbol{H} / 2) x^{m} / x^{3}$ and the above action becomes $S_{2}=-i\langle\boldsymbol{\sigma}\rangle \cdot \mathbf{g}$. Only place where 
the $\boldsymbol{\sigma}$ expectation value appears is the exponential $e^{-S_{2}}$ of the monopole effective action. Since the magnetic charge $\mathbf{g}$ is quantized in $\boldsymbol{\beta}_{a}^{*}, a=0,1,2 \ldots, r^{\prime}$, the scalar field $\boldsymbol{\sigma}$ would be physically equivalent to $\boldsymbol{\sigma}+2 \pi \boldsymbol{w}_{a}, a=0,2 \ldots r^{\prime}$, where $\boldsymbol{w}_{0}$ is the fundamental weight vector for the root $\boldsymbol{\beta}_{0}$. By going case by case, one can show that the fundamental cell of $\boldsymbol{\sigma}$ for the case with twisted boundary condition is identical to that of $\varphi$ as given in Eqs. (2.20) and (2.21). For the theory with non simply laced group, the $\boldsymbol{\varphi}$ and $\boldsymbol{\sigma}$ have the different ranges [19]. The general range of $\boldsymbol{\varphi}$ in Eq. (2.24) is translated to the range for the complex vector field $\boldsymbol{z}=i(\tau \boldsymbol{\varphi}+\boldsymbol{\sigma})$ as

$$
\boldsymbol{z} \in \mathcal{M}_{\text {vacuum }}=\frac{C^{r^{\prime}}}{\Lambda^{c} \rtimes W_{\mathcal{G}_{0}}},
$$

where $\Lambda^{c}$ is a lattice defined by $2 \pi i\left(\tau \boldsymbol{w}+\boldsymbol{w}^{\prime}\right)$ where $\boldsymbol{w}, \boldsymbol{w}^{\prime}$ are vectors appeared in the allowed large gauge transformations (2.18). For the twisted Lie algebra with classical groups, the complex $\boldsymbol{z}$ denotes the positions of D2 branes in the dual torus in the M-theory as we will see in the next section.

Including the dual photon contribution $S_{2}$ of Eq. (3.11) and the topological term $\frac{-i \theta}{4 \pi^{2}} \int_{S^{2}} d x^{m} \boldsymbol{\varphi} \mathbf{B}_{m}$, the Euclidean action for each fundamental monopole can be calculated for a given asymptotic value of the complex field $\boldsymbol{z}=i(\tau \boldsymbol{\varphi}+\boldsymbol{\sigma})$. Their actions (3.8) become generalized to holomorphic ones,

$$
-S_{0}=\frac{4 \pi i \tau}{L \boldsymbol{\beta}_{0}^{2}}+\boldsymbol{\beta}_{0}^{*} \cdot\langle\boldsymbol{z}\rangle, \quad-S_{a}=\boldsymbol{\beta}_{i}^{*}\langle\boldsymbol{z}\rangle,
$$

which give the exponential suppressing factors as the real value of the negative action is negative.

In the small radius limit, where $m^{-1} \ll R \ll \Lambda_{C D R}^{-1}$, one can in principle calculate the contribution to the superpotential by fundamental monopoles by calculating the massless gluino correlation functions. Following the argument in Ref. [18], we choose the running coupling to be given at the mass scale $m$, and replace the expectation value $\langle\boldsymbol{z}\rangle$ by the dynamical chiral field $\boldsymbol{X}$. As we consider the $\mathcal{N}=1^{*}$ theory, which is finite at high energy, the regularization should be somewhat different from that of the Pauli-Villars regularization. The calculation would be more or less identical to that in Ref. 18] and we obtain the leading contribution to the effective potential $(2.30)$ as

$$
\mathcal{W}_{\mathcal{N}=1}^{\text {twisted }}=m^{3}\left(\frac{2}{\boldsymbol{\beta}_{0}^{2}} e^{\frac{4 \pi i \tau}{L \boldsymbol{\beta}_{0}^{2}}+\boldsymbol{\beta}_{0}^{*} \cdot \boldsymbol{X}}+\sum_{a=1}^{r^{\prime}} \frac{2}{\boldsymbol{\beta}_{a}^{2}} e^{\boldsymbol{\beta}_{a}^{*} \cdot \boldsymbol{X}}\right)
$$

This is the holomorphic superpotential for the $\mathcal{N}=1$ supersymmetric theory with twisted boundary condition. The above superpotential is the affine Toda potential 
for the associated affine algebra. It is independent of the compactification radius $R$ and so should be true for any value of $R$ due to holomorphic property. Following Ref. 18] one can calculate the vacuum degeneracy and the gluino condensation at each vacua by finding the stationary points of the above potential. In the infinite radius limit, the physics should be identical whether one uses periodic or twisted boundary condition. Indeed one can easily show that this is true due to the identities enlisted in Appendix E for roots and comarks of the untwisted and twisted affine algebra. We will work out this physics in detail by using the glueball superpotential in Sec.6, where the above affine Toda potential will be mapped to the Veneziano-Yankielowicz superpotential.

\subsection{Superpotential: Summing Over Higher Instantons}

For $\mathcal{N}=1^{*}$ theory, all other magnetic monopoles also seem to contribute to the superpotential [20]. Noting that the step operator for the root $(\beta, m+n / L)$ and that for the root $(-\boldsymbol{\beta},-m-n / L)$ lead to the gauge equivalent monopole solutions, we can restrict to the positive roots $\boldsymbol{\beta}$ in calculating the monopole instanton contribution. Their contribution to the negative action $-S(m, n)$ with $m \in Z$ and $n=0,1, \ldots(L-1)$ can be divided into two groups as follows,

$$
\begin{aligned}
& -S_{m, s}^{+}=2 \pi i \tau \frac{2 m}{\boldsymbol{\beta}^{2}}+\left(2 \pi i \tau \frac{2 n}{\boldsymbol{\beta}^{2} L}+\boldsymbol{\beta}^{*} \cdot\langle\boldsymbol{z}\rangle\right), \quad(m \geq 0, L-1 \geq n \geq 0) \\
& -S_{m, s}^{-}=2 \pi i \tau \frac{2 m}{\boldsymbol{\beta}^{2}}-\left(2 \pi i \tau \frac{2 n}{\boldsymbol{\beta}^{2} L}+\boldsymbol{\beta}^{*} \cdot\langle\boldsymbol{z}\rangle\right), \quad(m \geq 1, L-1 \geq n \geq 0)
\end{aligned}
$$

As in for $S U(r+1)$ theory studied in Ref. [20], we also expect the contribution from the $k$ number of every $(\boldsymbol{\beta}, m+n / L)$ monopole instantons whose action would be $k$ times that of $(\boldsymbol{\beta}, m+n / L)$ monopole. Thus in the semiclassical approximation, the most general possible holomorphic superpotential generated by the $(\boldsymbol{\beta}, m+n / L)$ monopoles with all possible $m$ would be

$$
\mathcal{W}(\boldsymbol{\beta}, n / L)=\sum_{k=1}^{\infty} a_{k} y^{k}+\sum_{k=1}^{\infty} \sum_{m=1}^{\infty}\left(q^{\frac{2}{\beta^{2}}}\right)^{k m}\left(b_{k, m} y^{k}+c_{k, m} y^{-k}+d_{k, m}\right)
$$

where

$$
q=e^{2 \pi i \tau}, \quad y=e^{\frac{4 \pi i \tau n}{\boldsymbol{\beta}^{2} L}+\boldsymbol{\beta}^{*} \cdot \boldsymbol{X}} .
$$

The direct calculation of the exact coefficients of these contribution would be a challenge. The $y$ independent contributions would be pure instanton contributions.

Fortunately, for the $A_{r}=S U(r+1)$ gauge theory with periodic boundary condition, the above contribution of all magnetic monopoles seems to be summed to a 
Weierstrass elliptic function, which is justified from other considerations [20]. Here we also assume that a similar replacement does the trick. Thus, the above action for the root $(\boldsymbol{\beta}, n / L)$ of $\mathcal{G}_{n}$ becomes

$$
\mathcal{W}(\boldsymbol{\beta}, n / L) \sim \wp\left(\boldsymbol{\beta}^{*} \cdot \boldsymbol{X}+2 \pi i \tau \frac{2 n}{\boldsymbol{\beta}^{2} L} ; 2 \pi i, 2 \pi i \tau \frac{2}{\boldsymbol{\beta}^{2}}\right) .
$$

To fix the various terms, we have used the series expansion (F.4) of the Weierstrass elliptic function in Appendix F. There will be one such expression for all positive roots in the $\mathcal{G}_{n}$. In summing over the contribution for each positive root, we fix the coefficient of the elliptic function, by considering the contributions from single fundamental monopole instanton given in Eq. (3.14). Here it is also useful to consider the series expansion of the Weierstrass elliptic function in Appendix F. In addition, we put the same coefficient to the roots of the same length for Weyl reflection symmetries. Then, we obtain a preliminary holomorphic superpotential (2.30) for the $\mathcal{N}=1^{*}$ theory on $R^{1+2} \times S^{1}$ with twisted boundary condition as

$$
\tilde{\mathcal{W}}_{\mathcal{G}^{(L)}}=m^{3} \sum_{n=0}^{L-1} \sum_{\boldsymbol{\beta} \in \mathcal{R}_{+}\left(\mathcal{G}_{n}\right)} \frac{2}{\boldsymbol{\beta}^{2}} \wp\left(\boldsymbol{\beta}^{*} \cdot \boldsymbol{X}+2 \pi i \tau \frac{2 n}{L \boldsymbol{\beta}^{2}} ; 2 \pi i, 2 \pi i \tau \frac{2}{\boldsymbol{\beta}^{2}}\right)
$$

where $\mathcal{R}_{+}\left(\mathcal{G}_{n}\right)$ is the set of positive roots in $\mathcal{G}_{n}$. Note that this potential is even under $\boldsymbol{X} \rightarrow-\boldsymbol{X}$ due to the summation and so the above sum could be extended to the sum over all roots in $\mathcal{G}_{n}$ once the factor half is included. As we choose the same normalization for equal length roots, the Weyl reflection symmetry is manifest. This contrasts to the effective potential (3.14) for the $\mathcal{N}=1$ theory where the Weyl reflection symmetry is already used to put $\boldsymbol{X}$ in the fundamental alcove.

Here we present for the sake of completeness the $\mathcal{N}=1^{*}$ effective superpotential for the $\mathcal{N}=1^{*}$ theory of a simple Lie algebra $\mathcal{G}$ with periodic boundary condition, which is

$$
\mathcal{W}_{\mathcal{G}^{(1)}}=m^{3} \sum_{\boldsymbol{\alpha} \in \mathcal{R}_{+}(\mathcal{G})} \frac{2}{\boldsymbol{\alpha}^{2}} \wp\left(\boldsymbol{\alpha}^{*} \cdot \boldsymbol{X} ; 2 \pi i, 2 \pi i \tau \frac{2}{\boldsymbol{\alpha}^{2}}\right)
$$

For $A_{r}=S U(r+1)$, we recover the Dorey's result 20]. This superpotential for the untwisted group $\mathcal{G}^{(1)}$ can be recognized to be related to the twisted elliptic CalogeroMoser model for the Lie group $\mathcal{G}$. They have appeared as the integrable model related to the Seiberg-Witten curve for the $\mathcal{N}=2^{*}$ theory of the gauge algebra $\mathcal{G}$ [11]. Also they appear in the study of the relation between the integrable model and the $\mathcal{N}=1^{*}$ theory [21]. Contrast to these works, our result has definite normalization for the superpotential. This will allow the study of the modular property of the effective superpotential in more detail. 
Let us now consider the three dimensional limit of $\mathcal{N}=1^{*}$ theories as done for the $S U(r)$ case in Ref. [20]. We take a small $R$ limit so that $R^{-1}>m^{-1}$, keeping the three dimensional coupling constant $2 \pi R / e^{2}(m)=g_{3}^{2}$ fixed, which means a small coupling or small $q$ limit. The corresponding three dimensional effective potential would be $\mathcal{W}_{3}=2 \pi R \cdot \mathcal{W}$. With periodic boundary condition, the three dimensional gauge group would remain $\mathcal{G}$ and all $q$-dependence disappears. ¿From the formula in Appendix F, we can read

$$
\mathcal{W}_{3}(\mathcal{G})=2 \pi R m^{3} \sum_{\alpha \in \mathcal{R}_{+}(\mathcal{G})} \frac{2}{\boldsymbol{\alpha}^{2}} \frac{1}{\sinh ^{2}\left(\frac{\boldsymbol{\alpha} \cdot X}{2}\right)} .
$$

(Here to achieve the three dimensional limit one should rescale the mass parameter to absorb the length scale $R$.) We expect the three dimensional limit of the theories with twisted boundary condition leads to the $\mathcal{N}=1^{*}$ theory with gauge group $\mathcal{G}_{0}$. Indeed in this limit all instanton contribution from the twisted sectors $\mathcal{G}_{n}, n \neq 0$ disappear. The three dimensional limit of the superpotential (3.21) for the non simply laced group $\mathcal{G}$ becomes identical to the above expression (3.22) once the three dimensional algebra $\mathcal{G}_{0}$ is identified with the non simply laced Lie algebra $\mathcal{G}$. This is a consistency check for our effective superpotential.

\section{4. $S L(2, Z)$ Symmetry}

\section{1 $S L(2, Z)$ and Four Types of Orientifold Planes}

The $\mathcal{N}=4$ supersymmetric Yang-Mills theory with $S U(r)$ symmetry on $R^{1+3}$ dimension describes the low energy dynamics of $r$ parallel D3 branes of the type IIB theory when their mutual distance is smaller than the string length. The type IIB string theory has the $S L(2, Z)$ symmetry and $D 3$ branes are invariant under it. This symmetry manifest nonpertubratively in the $\mathcal{N}=4$ supersymmetric gauge theory on $D 3$ branes as a generalization of weak-strong coupling duality.

To introduce the other classical groups on $R^{1+3}$, we need to introduce the threedimensional orientifold parallel to D3 branes. (See Ref. [36] for a review.) There are four kinds of them, $\mathrm{O3}^{-}, \mathrm{O3}^{+}, \widetilde{\mathrm{O3}}^{+}, \widetilde{\mathrm{O3}}^{-}$, whose low energy field theory with $r$ parallel $D 3$ branes has gauge algebra, $D_{r}, C_{r}, C_{r}$ and $B_{r}$, respectively. Under the $S$-duality two orientifolds $O 3^{-}$and $\widetilde{O 3}^{+}$are invariant and two orientifolds $\mathrm{O3}^{+}$and $\widetilde{O 3}^{-}$are interchanged. Under the $T$ transformation of the $S L(2, Z)$, two orientifolds $\mathrm{O3}^{+}$and $\widetilde{\mathrm{O3}}^{+}$are interchanged.

Perhaps not widely recognized, although elementary, is the nature of two $S p(r)$ theories out of orientifold construction. We know that there exists only one $\mathcal{N}=4$ 
$S p(r)$ Yang-Mills theory in four dimensions, so the question is what are the distinction between the two + type orientifolds in terms of the field theory.

The answer lies in the $\theta$ angle. In string theory convention in $3+1$ dimension, the long roots of the $C_{r}=S p(r)$ theory have length square four and so the range of $\theta$ is increased to $4 \pi$ instead of $2 \pi$. On the other hand, the $\theta$ angle of $\mathcal{N}=4$ theory is inherited from the type IIB axion which has a natural period $2 \pi$. Thus, there is an apparent discrepancy of the period between type IIB picture and Yang-Mills picture.

However, when we say IIB axion having period $2 \pi$, it actually means that $T$ transformation of IIB $S L(2, Z)$ shifts the axion by $2 \pi$. Since both $O 3^{+}$and $\widetilde{O 3}^{+}$has a discrete $Z_{2}$ NS-NS 2-form flux and since $\widetilde{O 3}^{+}$has additional RR 2-form flux, also $Z_{2}$ valued, the same $T$ transformation of IIB interchanges these two orientifolds and at the same time shifts the axion by $2 \pi$. From this, it is clear how the $4 \pi$ range of $\theta$-angle is generated from IIB viewpoint. Half of this $4 \pi$ range is covered by $\mathrm{O}^{+}$and the other half by $\widetilde{O 3}^{+}$. There is no fundamental distinction between the two cases, since by continuous deformation of IIB axion combined with $T$ transformations, the two can be mapped to each other.

\subsection{Compactification and M-theory Realization}

Another way to view the $S L(2, Z)$ symmetry of IIB theory and in particular the $S L(2, Z)$ of four dimensional Yang-Mills theory is to rely on M theory. The type IIB string theory on flat 10 dimension has the $S L(2, Z)$ symmetry. This symmetry can be understood from the $M$ theory point of view by considering the compactification of type IIB theory on a circle of radius $R$ along the $x^{4}$ coordinate, which is T-dual to the type IIA theory on a dual circle of radius $1 / R$. Type IIA theory on flat 10 dimensional space can be obtained from compactifying the $\mathrm{M}$ theory on a circle along $x^{11}$ coordinate. Thus the $\mathrm{M}$ theory on two torus $T^{2}$ is T-dual to the type IIB theory on a circle. The obvious $S L(2, Z)$ symmetry of the torus compactification of the $M$ theory is translated to the $S L(2, Z)$ symmetry of type IIB theory on a circle [37.

A flat M2 brane located on a point on $T^{2}$ would be a D2 brane on type IIA theory, which is a $T$-dual of a D3 brane wrapped on the circle. The M2 brane does not change under the $\mathrm{SL}(2, \mathrm{Z})$ of the torus and so the wraped D3 branes is also a $S L(2, Z)$ singlet of type IIB as we know well. We regard the circle along $x^{4}$ as the compact circle of our spacetime $R^{1+2} \times S^{1}$. If we have $r$ parallel D3 branes wrapping the circle along $x^{4}$ and lying very close to each other in the transverse direction, the effective low energy field would be the $\mathcal{N}=4$ supersymmetric field theory on $R^{1+2} \times S^{1}$ with gauge group $S U(r)$. The expectation value $\langle\boldsymbol{\sigma}\rangle$ of the dual 

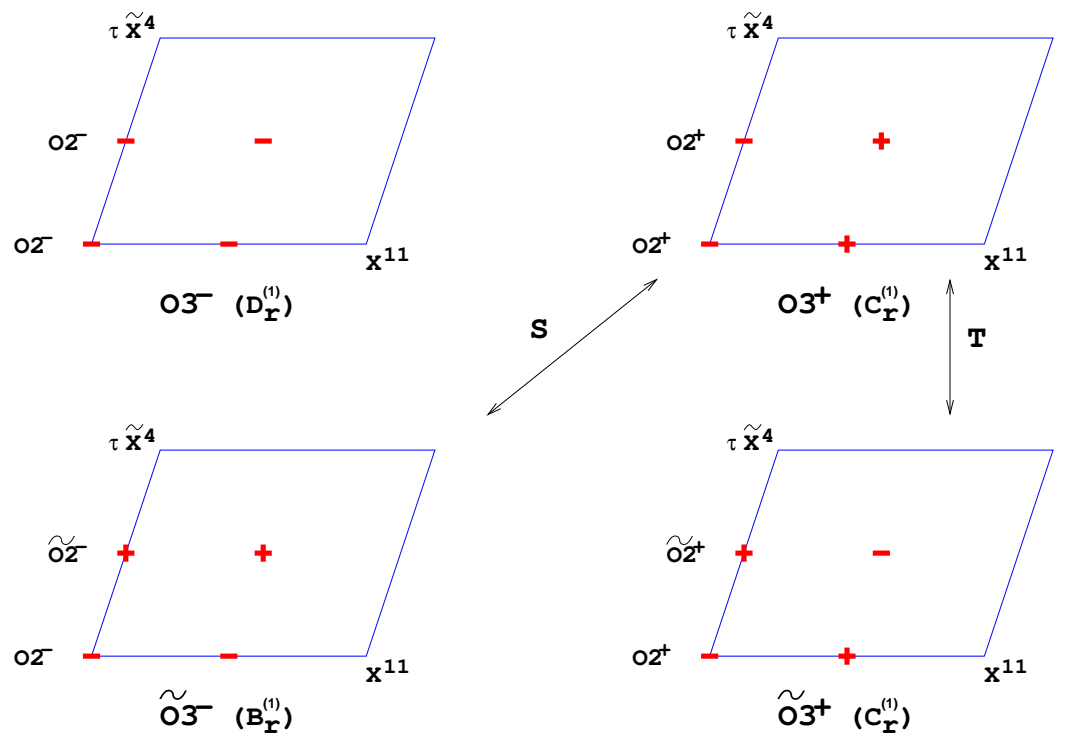

Figure 1: The four diagrams show $M$ theory realization of four types of $O 3$ planes compactified on a circle. After a T-duality, which splits a single $O 3$ to a pair of $O 2$ 's, we lift the configurations to $\mathrm{M}$ theory by adding a $x^{11}$ circle. Each $O 2$ is composed of two $O M 2$ 's, separated along $x^{11}$, of which there are two types, $O M 2^{+}$and $O M 2^{-}$. These are denoted by red +'s and red -'s on the torus. The four dimensional limit corresponds to shrinking the torus while maintaining its complex modulus fixed.

photon shows the location of the $M 2$ branes along $x^{11}$, and the expectation value $\langle\boldsymbol{\varphi}\rangle$ shows the position of $M 2$ branes along the dual $\tilde{x}^{4}$ direction. As there is nonzero $\theta$ parameter, two directions are not orthogonal but skewed and $\langle\boldsymbol{z}\rangle=\langle\tau \boldsymbol{\varphi}+\boldsymbol{\sigma}\rangle$ denotes the complex positions of $M 2$ branes on the two torus. In Figure 1, we draw the two torus $T^{2}$ along dual $\tilde{x}^{4}$ and $x^{11}$ direction in the M-theory. When we take the infinite radius limit, the dual two torus $T^{2}$ shrinks and so the $S L(2, Z)$ symmetry will still hold.

We can add an O3 plane to D3 branes to get other classical gauge groups in four dimension. The four kinds of O3 orientifolds lifted to M theory has been fairly well discussed in Ref. 38 and here we summarize it briefly for the completeness. Upon compactification, we wrap them along the $x^{4}$ circle. After T-duality a $O 3$ plane breaks to two $\mathrm{O} 2$ planes on the dual $\tilde{x}^{4}$ in the type IIA theory which makes the dual circle to be a line segment. There are four kinds of O3 orientifolds as well as there are four kinds of two dimensional planes $\mathrm{O}^{-}, \mathrm{O}^{+}, \widetilde{\mathrm{O}}^{+}, \widetilde{\mathrm{O}}^{-}$. Figure 1 shows that how four $O 3$ planes are decomposed to two $O 2$ planes lying along the dual $\tilde{x}^{4}$ direction. From the M-theory point of view a single two dimensional orientifold is made of two OM2 planes lying along the $x^{11}$ direction. There are $O M 2^{+}$and $O M 2^{-}$ planes. Thus a single $O 3$ plane is made of four OM2 planes on a torus. 
Figure 1. also shows this M theory lift of the four kinds of O3 branes. The two signs denote the two possible OM planes. The complex structure of $T^{2}$ becomes the coupling constant $\tau$. The lattice of $O M$ planes on the $T^{2}$ could have some obvious symmetries. The point to notice is that $\mathrm{O3}^{+}$and $\widetilde{\mathrm{O3}}{ }^{-}$are S-dual to each other, $\widetilde{O 3}^{+}$ are self S-dual, and $O 3^{-}$are $S L(2, Z)$ symmetric, regardless of the shape of $T^{2}$ or the $\tau$ coupling constant. In addition the $T$ of the $S L(2, Z)$ transforms $O 3^{+}$to $\widetilde{O 3}^{+}$. ¿From the picture we can see the $S L(2, Z)$ of the $O 3^{-}$configuration or $D_{r}$ theory as expected. Also we can see the $\Gamma_{0}(2)$ symmetry for the rest of $O 3$ planes. This is the $M$ theory picture of the $\Gamma_{0}(2)$ theory for the $\mathcal{N}=4$ supersymmetric theories with $B_{r}, C_{r}$ gauge groups and periodic boundary condition. As argued before these modular symmetry also appear in the superpotential for the $\mathcal{N}=1^{*}$ theory with classical groups and periodic boundary condition.

\subsection{Theory Realization of Twisted Theories}

Let us now consider the $\mathcal{N}=4$ supersymmetric Yang-Mills theory on $R^{1+2} \times S^{1}$ with twisted boundary condition. One can read off the T-dual picture of the brane configuration. We need two O2 planes and D2 branes along the dual circle, which can be determined from the extended Dynkin diagram. The $A_{2 r}^{(2)}$ theory arises from the low energy theory on one $\widetilde{O 2}^{-}$plane and one $\mathrm{O}^{+}$or $\widetilde{\mathrm{O}}^{+}$plane with $\mathrm{D} 2$ branes inserted between them. The $A_{2 r-1}^{(2)}$ theory arises from the low energy theory on one $O 2^{-}$plane and one $O 2^{+}$or $\widetilde{O}^{+}$plane. The $D_{r+1}^{(2)}$ theory arises from the low energy theory on one $\widetilde{O 2}^{-}$plane and one $\widetilde{O}^{-}$plane with D2 branes inserted between them.

Their corresponding M-theory pictures on $T^{2}$ are shown in Figure 2. One can see that the $S L(2, Z)$ symmetry manifests for these twisted case with arbitrary coupling constant $\tau$. Thus, the corresponding $\mathcal{N}=4$ theory with twisted boundary condition are expected to have the exact $S L(2, Z)$ symmetry. Similar stringy realization of the $\mathcal{N}=4$ supersymmetric models in various dimensions with twisted boundary condition has been realized in Ref. [22, 39].

This fact that all twisted $(N=4)$ theories are invariant under $S L(2, Z)$ may be quite surprising, since none of the extended Dynkin diagrams in question are simplylaced. On the other hand, note that all twisted theories arise from twisting a gauge theory with simply-laced gauge groups, namely $A_{r}, D_{r}$, and $E_{6}$, which, prior to the twisting, are all equipped with $S L(2, Z)$. The twisting (upon compactification) must commute with the action of $S L(2, Z)$ for four dimensional theories. We do not have a concrete understanding of why this is so in the full string theory setting. 

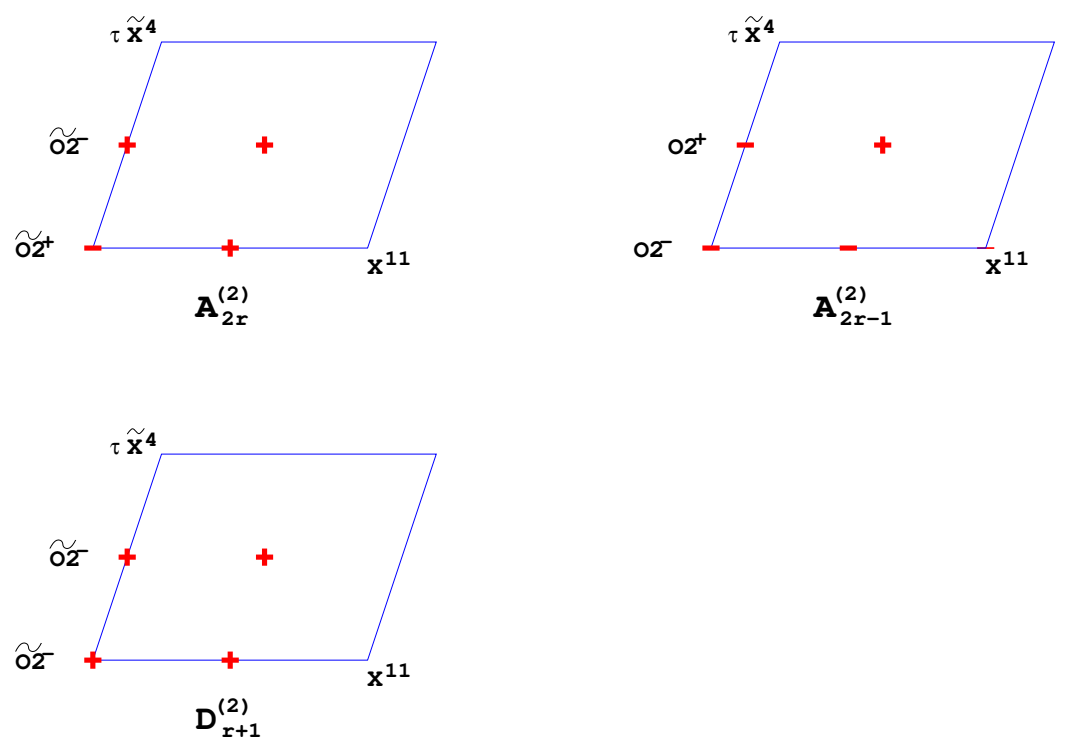

Figure 2: $\mathrm{M}$ theory realization of $O 2$ planes that gives the twisted theories with classical groups upon T-duality. Again each $O 2$ is composed of two $O M 2$ 's, separated along $x^{11}$, of which there are two types, $O M 2^{+}$and $O M 2^{-}$, denoted by red +'s and red -'s on the torus. Together with four possibilities of Figure 1, these exhaust all possible such combination of $O M 2$ 's up to overall translations of the torus.

\section{5. $S L(2, Z)$ Symmetries and Exact Superpotential}

In this work we are searching for the superpotential for the $\mathcal{N}=1^{*}$ theory with twisted boundary condition, starting from that for the $\mathcal{N}=1$ theory. While one does not have exact control over the multi-monopole instanton contributions, one may expect that the resulting effective superpotential for each class of magnetic charge would be expressible by Weierstrass elliptic function as in the $S U(N)$ gauge group case, which was roughly where we stopped in section 3 . While the resulting superpotential (3.20) has the right weak coupling limit to the $\mathcal{N}=1$ theory, there exists some ambiguity.

Our guess (3.20) for the superpotential of the $\mathcal{N}=1^{*}$ theory with twisted boundary condition has been shown to be consistent with the Weyl reflection, the three dimensional limit, and the week coupling limit or the affine-Toda limit. Hence one may conclude our guess is the right one. However, this is too hasty a conclusion. We will see our guess is almost but not quite right. When we convert the sum of the monopole contributions to a Weierstrass function, an ambiguity could arise from the uncertainty of the pure instanton effect which depends only on $q=e^{2 \pi i \tau}$. This does not affect the above consistency checks.

On the other hand, as we reviewed in the previous section, there is a strong indi- 
cation for the $S L(2, Z)$ duality of $\mathcal{N}=4$ theories which would be inherited by $\mathcal{N}=1^{*}$ theories. When we take into account the $S L(2, Z)$ symmetry, the purely instantonic ambiguity is resolved and we arrive at the somewhat modified new conjecture. This new conjecture is unique and also passes an additional consistency check given in the next section. In the next section the weak coupling expansion of the glueball superpotential will be obtained by the "dual" or "mirror" transformation. When the rank of the gauge group is small, the pure instanton contributions appear quickly in a few leading terms in the weak coupling series, and are playing a crucial role for this consistency.

\begin{tabular}{|c|c|}
\hline gauge group and boundary condition & effective superpotential \\
\hline$A_{r}^{(1)}, D_{r}^{(1)}, E_{6}^{(1)}, E_{7}^{(1)}, E_{8}^{(1)}$ & untwisted $A_{r}, D_{r}, E_{6}, E_{7}, E_{8} \quad \mathrm{eCM}$ \\
\hline$B_{r}^{(1)}, C_{r}^{(1)}, G_{2}^{(1)}, F_{4}^{(1)}$ & twisted $B_{r}, C_{r}, G_{2}, F_{4}$ eCM \\
\hline$A_{2 r}^{(2)}$ & twisted $B C_{r}$ eCM \\
$A_{2 r-1}^{(2)}$ & untwisted $C_{r}$ eCM \\
$D_{r+1}^{(2)}$ & untwisted $B_{r} \mathrm{eCM}$ \\
$E_{6}^{(2)}$ & untwisted $F_{4} \mathrm{eCM}$ \\
$D_{4}^{(3)}$ & untwisted $G_{2}$ eCM \\
\hline
\end{tabular}

Table I: The gauge group and boundary condition of a $\mathcal{N}=1^{*}$ theory on $R^{1+2} \times S^{1}$ and the corresponding elliptic Calogero-Moser Model.

At the end of day, we are able to modify the above conjecture (3.20) of the superpotential in such a way that it respects the $S L(2, Z)$ symmetry. The modification involves an analytic function of $q=e^{2 \pi i \tau}$ only, which can be attributed to the pure instanton contributions to the superpotential. Thus one could say the magnetic monopole contribution written in the Weierstrass function as in the previous section is fine but there was an ambiguity in the pure instanton contribution, which will be fixed by the $S L(2, Z)$ symmetry. The resulting effective superpotential will turn out to be those associated with the potential of certain elliptic Calogero-Moser model [40] with well defined coefficients. The following table I shows the detailed correspondence.

\section{$5.1 S L(2, Z)$ on Charge Lattice and Modular Transformation}

In the first subsection, we will briefly review how $S L(2, Z)$ acts on $\mathcal{N}=1^{*}$ theories on $S^{1}$ with untwisted boundary conditions. Apart from reviewing known material 
and also reinforce the inheritance of $S L(2, Z)$ by $\mathcal{N}=1^{*}$ theories, this will also identify the right modular transformation on the $\mathcal{N}=1^{*}$ fields. From the second subsections, we will turn to twisted cases.

The electromagnetic duality in the $\mathcal{N}=4$ supersymmetric field theory of Lie algebra $\mathcal{G}$ on $R^{1+3}$ is simplest to see from the electromagnetic charge spectrum of the theory when the gauge symmetry is maximally broken by adjoint scalar fields. In fact, the only solid evidence of $S L(2, Z)$ for $\mathcal{N}=4$ theories comes from checking that the actual spectrum of solitons does respect this symmetry, which consists of searching for and determining degeneracy of BPS states of monopoles and dyons. Checking the quantum spectrum explicitly was done in some simpler settings [41], and the result is consistent with the $S L(2, Z)$ symmetry. ${ }^{4}$

For simplicity, let us consider the case with a single adjoint scalar field breaking the gauge symmetry [42]. For any simple root $\boldsymbol{\alpha}_{a}$, the complex charge lattice is given by

$$
p_{a} \boldsymbol{\alpha}_{a}+\tau g_{a} \boldsymbol{\alpha}_{a}^{*}
$$

where electric charge $p_{a}$ and magnetic charge $g_{a}$ are integers defining a BPS state and its mass would be

$$
\sqrt{\frac{4 \pi}{\operatorname{Im} \tau}}\left|\left(p_{a} \boldsymbol{\alpha}_{a}+\tau g_{a} \boldsymbol{\alpha}_{a}^{*}\right) \cdot \boldsymbol{v}\right|,
$$

where $\boldsymbol{v}$ is the expectation value of the Higgs field whose kinetic energy is in the standard form without $1 / e^{2}$ factor. Here the magnetic charge $g_{a}$ is an integer as the soliton solution has integer topology. The electric charge $p_{a}$ is an integer due to the quantization.

The above charge lattice and mass for the theories with simply laced groups like $A_{r}, D_{r}, E_{6}, E_{7}, E_{8}$ are invariant under the $S L(2, Z)$ transformation of the coupling (2.10) and the charge lattice

$$
\left(\begin{array}{l}
g_{a} \\
p_{a}
\end{array}\right) \rightarrow\left(\begin{array}{ll}
a & c \\
b & d
\end{array}\right)^{-1}\left(\begin{array}{l}
g_{a} \\
p_{a}
\end{array}\right),
$$

where $a, b, c, d \in Z$ such that $a d-b c=1$.

The charge lattice for a non simply laced algebra is invariant under only a subgroup of the $S L(2, Z)$. As shown in Ref.42, the symmetry of the charge lattice for the theories of $B_{r}, C_{r}, F_{4}$ is $\Gamma_{0}(2)$, and that for the $G_{2}$ theory is $\Gamma_{0}(3)$, where $\Gamma_{0}(n)$ is

\footnotetext{
${ }^{4}$ The emergence of such spectra is not obvious at all. For instance, pure $\mathcal{N}=2$ theories share essentially the same classical BPS spectra with $\mathcal{N}=4$ theories [43], yet counting of quantum BPS states has shown the $\mathcal{N}=2$ spectra are almost never invariant under the $S L(2, Z)$ [44.
} 
the subalgebra such that $c=0 \bmod n$. This can be checked easily by considering the generators of $\Gamma_{0}(n)$. The reduced modular symmetry $\Gamma_{0}(2)$ for the $\mathcal{N}=4$ theories with gauge group $B_{r}, C_{r}$ is identical to that obtained from the M-theory picture in the previous section. One may expect this symmetry is exact for the $\mathcal{N}=4$ supersymmetric Yang-Mills theories with non simply laced algebra, which would imply many nontrivial predictions on magnetic monopole spectrum.

We note that there is an interesting aspect of this $\Gamma_{0}(2)$ symmetry. For the theories with $B_{r}, C_{r}, F_{4}$ gauge group, there are roots of two different lengths and the two length differs by $\sqrt{2}$. Take the example of $B_{r}$ where,

$$
\boldsymbol{\alpha}_{s}^{2}=1, \quad \boldsymbol{\alpha}_{l}^{2}=2,
$$

so that

$$
\boldsymbol{\alpha}_{s}^{*}=2 \boldsymbol{\alpha}_{s}, \quad \boldsymbol{\alpha}_{l}^{*}=\boldsymbol{\alpha}_{l}
$$

If we shift $\tau$ by one half,

$$
\tau \rightarrow \tau+\frac{1}{2}
$$

and then the above charge lattice get shifted

$$
\left(p_{s}+g_{s}\right) \boldsymbol{\alpha}_{s}+(2 \tau) g_{s} \boldsymbol{\alpha}_{s}, \quad\left(p_{l}+\frac{g_{l}}{2}\right) \boldsymbol{\alpha}_{l}+(2 \tau) \frac{g_{l}}{2} \boldsymbol{\alpha}_{l},
$$

with mutually co-prime integer pairs $\left(p_{s}, g_{s}\right)$ and $\left(p_{l}, g_{l}\right)$. This shifted charge lattice is easily seen to be invariant under a S-duality transformation

$$
2 \tau \rightarrow-\frac{1}{2 \tau}
$$

which interchanges between integer (half-integer) electric and integer (half-integer) magnetic charge of short (long) roots in $2 \tau$ unit. The superpotential for the corresponding $\mathcal{N}=1^{*}$ theory on $R^{1+2} \times S^{1}$ with periodic boundary condition has modular weight two once we also transform

$$
\boldsymbol{X} \rightarrow \frac{\boldsymbol{X}}{2 \tau}
$$

This self-duality is not new. One can show that the sequence of the transformations $\tau \rightarrow \tau+1 / 2,2 \tau \rightarrow-1 / 2 \tau$ and $\tau \rightarrow \tau-1 / 2$ belongs to the group $\Gamma_{0}(2)$ because of the relation

$$
\left(\begin{array}{ll}
1 & \frac{1}{2} \\
0 & 1
\end{array}\right)\left(\begin{array}{cc}
0 & -\frac{1}{2} \\
2 & 0
\end{array}\right)\left(\begin{array}{cc}
1 & -\frac{1}{2} \\
0 & 1
\end{array}\right)=\left(\begin{array}{ll}
1 & -1 \\
2 & -1
\end{array}\right)=S T^{-1} S T S^{-1} .
$$

Here $S$ and $T$ are some $S L(2, Z)$ generators such that $a=d=0$ and $-b=c=1$ for $S$, and $a=b=d=1$ and $c=0$ for $T$. A similar construction holds for $C_{r}$ and $F_{4}$. 
For $C_{r}$, this is precisely the " $S$ " transformation that leaves $\widetilde{O 3}{ }^{+}$orientifold invariant. The theory with $G_{2}$ gauge group has a similar transformation in $\Gamma_{0}(3)$.

In addition, the $S$-duality between the $\mathcal{N}=4$ theories of $B_{r}$ and $C_{r}$ gauge groups would be clear when one uses the normalization for the untwisted $C_{r}$ case such that its long roots have length square 4 and its short roots have length square 2. This normalization fits well with the string theory point of view where the simple roots of length square two originate from the fundamental strings connecting D branes and the simple roots of different length come from the orientifolds at the boundary.

Now the $\mathcal{N}=2^{*}$ and $\mathcal{N}=1^{*}$ theories for non simply laced gauge groups should inherit this modular transformation properties under the subgroup of $S L(2, Z)$. Indeed the reduced modular symmetry for the integrable models associated with the $\mathcal{N}=2^{*}$ theories, noticed in Ref. [11], turns out to be identical to that of the charge lattice noticed above. Here, the Weierstrass function $\wp(z)=\wp(z ; 2 \pi i, 2 \pi i \tau)$ transforms as a modular function of weight two as shown in Appendix F. Depending on the Lie algebra $\mathcal{G}$, we need to consider a subgroup of $S L(2, Z)$ with accompanying the superfield transformation,

$$
\boldsymbol{X} \rightarrow \frac{\boldsymbol{X}}{c \tau+d} .
$$

When we compactify one of spatial directions, the charged particles have logarithmically divergent energy and so the charge lattice has no direct physical meaning, even though the $S L(2, Z)$ may remain meaningful. As the superpotential of the $\mathcal{N}=1^{*}$ theories with periodic boundary condition is characterized by the same integrable model as that of the corresponding $\mathcal{N}=2^{*}$ theories, the superpotential would have the same reduced modular properties. Indeed it is straightforward to see that the superpotential (3.21) for the untwisted theories with simply laced groups $A_{r}, D_{r}, E_{6}, E_{7}, E_{8}$ has the $S L(2, Z)$ modular symmetry. The superpotential (3.21) for the untwisted theories with gauge groups $B_{r}, C_{r}, F_{4}$ has the $\Gamma_{0}(2)$ modular symmetry. Finally the superpotential (3.21) for the untwised theory with $G_{2}$ gauge group has the $\Gamma_{0}(3)$ modular symmetry.

In addition the $S$-duality between the $\mathcal{N}=1^{*}$ theories of $B_{r}$ and $C_{r}$ gauge groups can be seen clearly in the string normalization. To see this in the effective superpotential (3.21), we rescale the parameters for $C_{r}$ case so that

$$
\sqrt{2} \boldsymbol{\alpha}, \sqrt{2} \boldsymbol{X}, 2 \tau, \mathcal{W} \rightarrow \boldsymbol{\alpha}, \boldsymbol{X}, \tau, \mathcal{W} / 2 .
$$

The newly normalized superpotential for the untwisted $C_{r}$ case becomes

$$
\mathcal{W}_{C_{r}^{(1)}}^{(n n)}=\sum_{\boldsymbol{\alpha} \in \mathcal{R}^{+}\left(\boldsymbol{\alpha}^{2}=2\right)} \wp(\boldsymbol{\alpha} \cdot \boldsymbol{X} ; 2 \pi i, 2 \pi i \tau)+\sum_{\boldsymbol{\alpha} \in \mathcal{R}^{+}\left(\boldsymbol{\alpha}^{2}=4\right)} \frac{1}{2} \wp\left(\frac{1}{2} \boldsymbol{\alpha} \cdot \boldsymbol{X} ; 2 \pi i, \pi i \tau\right),
$$


which is S-dual to the superpotential for the untwisted $B_{r}$ theory,

$$
\mathcal{W}_{B_{r}^{(1)}}=\sum_{\boldsymbol{\alpha} \in \mathcal{R}^{+}\left(\boldsymbol{\alpha}^{2}=2\right)} \wp(\boldsymbol{\alpha} \cdot \boldsymbol{X} ; 2 \pi i, 2 \pi i \tau)+\sum_{\boldsymbol{\alpha} \in \mathcal{R}^{+}\left(\boldsymbol{\alpha}^{2}=1\right)} 2 \wp(2 \boldsymbol{\alpha} \cdot \boldsymbol{X} ; 2 \pi i, 4 \pi i \tau),
$$

as $\mathcal{W}_{C_{r}^{(1)}}^{n n}(\boldsymbol{X} / \tau)=\tau^{2} \mathcal{W}_{B_{r}^{(1)}}(\boldsymbol{X})$. Here we dropped the mass parameter $m^{3}$ for simplicity.

\section{$5.2 A_{2 r}^{(2)}$ Case}

For $A_{2 r}^{(2)}$ affine algebra, the subalgebra $\mathcal{G}_{0}=B_{r}=S O(2 r+1)$ of even generators of $A_{2 r}$ have $r^{2}$ positive roots,

$$
\text { medium roots } \boldsymbol{\beta}_{m}=\frac{\boldsymbol{e}_{a} \pm \boldsymbol{e}_{b}}{\sqrt{2}}(a<b), \text { short } \operatorname{roots} \boldsymbol{\beta}_{s}=\frac{\boldsymbol{e}_{a}}{\sqrt{2}}
$$

with $\boldsymbol{e}_{a}(a=1, \ldots, r)$ being orthonormal vectors. The subset $\mathcal{G}_{1}$ of odd generators of $A_{2 r}^{(2)}$ belongs to the symmetric traceless representation of $\mathcal{G}_{0}$ and has $r^{2}+r$ positive root vectors,

$$
\text { long roots } \boldsymbol{\beta}_{l}=\sqrt{2} \boldsymbol{e}_{a}, \text { medium roots } \boldsymbol{\beta}_{m} \text {, short roots } \boldsymbol{\beta}_{s} .
$$

Clearly the root vectors of $\mathcal{G}_{1}$ is that of the so-called $B C$ system, which are made of the roots of $B_{r}$ and $C_{r}$ algebras.

Let us now express the superpotential $(3.20)$ for $A_{2 r}^{(2)}$ case. The contribution from the medium roots of even and odd parts is

$$
2 \sum_{\boldsymbol{\beta}_{m}}\left\{\wp\left(2 \boldsymbol{\beta}_{m} \cdot \boldsymbol{X} ; 2 \pi i, 4 \pi i \tau\right)+\wp\left(2 \boldsymbol{\beta}_{m} \cdot \boldsymbol{X}+2 \pi \tau ; 2 \pi i, 4 \pi i \tau\right)\right\}=2 \sum_{\boldsymbol{\beta}_{m}} \wp\left(2 \boldsymbol{\beta}_{m} \cdot \boldsymbol{X}\right),
$$

by the half period formula up to a $\tau$-only function. From now on the Weierstrass elliptic function $\wp(z ; 2 \pi i, 2 \pi i \tau)$ is denoted as $\wp(z)$ for simplicity. Similarly, the contributions from the short roots of even and odd parts become

$$
\begin{aligned}
& 4 \sum_{\boldsymbol{\beta}_{s}}\left\{\wp\left(4 \boldsymbol{\beta}_{s} \cdot \boldsymbol{X} ; 2 \pi i, 8 \pi i \tau\right)+\wp\left(4 \boldsymbol{\beta}_{s} \cdot \boldsymbol{X}+4 \pi i \tau ; 2 \pi i, 8 \pi i \tau\right)\right\} \\
& \quad=\sum_{\boldsymbol{\beta}_{s}} \wp\left(2 \boldsymbol{\beta}_{s} \cdot \boldsymbol{X} ; \pi i, 2 \pi i \tau\right)=\sum_{\boldsymbol{\beta}_{s}}\left(\wp\left(2 \boldsymbol{\beta}_{s} \cdot \boldsymbol{X}\right)+\wp\left(2 \boldsymbol{\beta}_{s} \cdot \boldsymbol{X}+\pi i\right)\right),
\end{aligned}
$$

by scaling and half-period formula up to a $\tau$-only function. The contribution from the long roots, which only comes from odd generators, is

$$
\sum_{\boldsymbol{\beta}_{l}} \wp\left(\boldsymbol{\beta}_{l} \cdot \boldsymbol{X}+\pi i \tau ; 2 \pi i, 2 \pi i \tau\right) .
$$


The potential with the general coefficients for long, short and shortest roots have appeared in Ref. [25, 26] as the twisted elliptic Calogero model for the $B C$ system.

Now putting all contributions together and noticing $2 \boldsymbol{\beta}_{s}=\boldsymbol{\beta}_{l}$, we obtain the following expression for the superpotential,

$$
\mathcal{W}_{A_{2 r}^{(2)}}=\sum_{\boldsymbol{\beta}_{m}} 2 \wp\left(2 \boldsymbol{\beta}_{m} \cdot \boldsymbol{X}\right)+\sum_{\boldsymbol{\beta}_{l}}\left(\wp\left(\boldsymbol{\beta}_{l} \cdot \boldsymbol{X}\right)+\wp\left(\boldsymbol{\beta}_{l} \cdot \boldsymbol{X}+\pi i\right)+\wp\left(\boldsymbol{\beta}_{l} \cdot \boldsymbol{X}+\pi i \tau\right)\right),
$$

where the sum is for all positive medium and long roots. Note that the funny looking range (2.20) and (3.12) of the bosonic part $\boldsymbol{z}$ of $\boldsymbol{X}$ in the $A_{2 r}^{(2)}$ theory is just suitable for the above expression for the superpotential as it is periodic under $\boldsymbol{\beta}_{l} \cdot \boldsymbol{z} \sim \boldsymbol{\beta}_{l} \cdot \boldsymbol{z}+\pi i \sim \boldsymbol{\beta}_{l} \cdot \boldsymbol{z}+\pi i \tau$. The Weyl reflection is manifest.

The $S L(2, Z)$ symmetry of the above potential can be seen as follows: the first two sums each have the right modular properties under the $S L(2, Z)$. For the rest, note that $S$, which maps $\tau \rightarrow-1 / \tau, \boldsymbol{X} \rightarrow \boldsymbol{X} / \tau$, exchanges the last two terms up to the modular transformation of weight two. On the other hand, $T$, which transforms $\tau \rightarrow \tau+1, \boldsymbol{X} \rightarrow \boldsymbol{X}+\pi i \sum_{a=1}^{r} \boldsymbol{e}_{a} / \sqrt{2}$, also interchanges the second and third expressions. Since $S$ and $T$ generate $S L(2, Z)$, the sum of the last three terms together have the right modular properties.

\section{$5.3 A_{2 r-1}^{(2)}$ Case}

For the $A_{2 r-1}^{(2)}$ case, the $\mathcal{G}_{0}=C_{r}=S p(r)$ of even generators has $r^{2}$ positive generators,

$$
\text { short roots } \boldsymbol{\beta}_{s}=\frac{\boldsymbol{e}_{a} \pm \boldsymbol{e}_{b}}{\sqrt{2}}(a<b), \quad \text { long roots } \boldsymbol{\beta}_{l}=\sqrt{2} \boldsymbol{e}_{a},
$$

where $\boldsymbol{e}_{a}$ with $a=1, \ldots, r$ are orthonormal vectors. The $\mathcal{G}_{1}$ of odd generators belongs to the $2 r^{2}+r$ dimensional representation and has $r^{2}-r$ positive root vectors,

$$
\text { short roots } \boldsymbol{\beta}_{s} \text {. }
$$

The contribution to the superpotential (3.20) from the monopole instantons associated with the long roots only in the $\mathcal{G}_{0}$ is

$$
\sum_{\boldsymbol{\beta}_{l}} \wp\left(\boldsymbol{\beta}_{l} \cdot \boldsymbol{X} ; 2 \pi i, 2 \pi i \tau\right) .
$$

The contribution to the superpotential from the monopole instantons associated with the short roots of the even and odd generators is

$$
\begin{aligned}
& 2 \sum_{\boldsymbol{\beta}_{s}}\left(\wp\left(2 \boldsymbol{\beta}_{s} \cdot \boldsymbol{X} ; 2 \pi i, 4 \pi i \tau\right)+\wp\left(2 \boldsymbol{\beta}_{s} \cdot x+2 \pi i \tau ; 2 \pi i, 4 \pi i \tau\right)\right) \\
& =2 \sum_{\boldsymbol{\beta}_{s}} \wp\left(2 \boldsymbol{\beta}_{s} \cdot \boldsymbol{X} ; 2 \pi i, 2 \pi i \tau\right),
\end{aligned}
$$


up to a $\tau$ dependent function after using the half-period formula. Thus, the holomorphic superpotential is

$$
\mathcal{W}_{A_{2 r-1}^{(2)}}=2 \sum_{\boldsymbol{\beta}_{s}} \wp\left(2 \boldsymbol{\beta}_{s} \cdot \boldsymbol{X}\right)+\sum_{\boldsymbol{\beta}_{l}} \wp\left(\boldsymbol{\beta}_{l} \cdot \boldsymbol{X}\right)
$$

where the sum is only for the positive roots. The $S L(2, Z)$ property is manifest. In addition we note that it is associated with untwisted elliptic Calogero-Moser model for the Lie algebra $B_{r}=S O(2 r+1)$, whose extended Dynkin diagram is the diagram for the simple coroots of $A_{2 r-1}^{(2)}$.

\section{$5.4 D_{r+1}^{(2)}$ Case}

For the $D_{r+1}^{(2)}$, the $\mathcal{G}_{0}=B_{r}$ of even generators has $r^{2}$ positive roots

$$
\text { long } \operatorname{roots} \boldsymbol{\beta}_{l}=\boldsymbol{e}_{a} \pm \boldsymbol{e}_{b}(a<b), \text { short } \operatorname{roots} \boldsymbol{\beta}_{s}=\boldsymbol{e}_{a}
$$

where $\boldsymbol{e}_{a}, a=1,2, \ldots, r$ are $r$ orthonormal vectors. The $\mathcal{G}_{1}$ of odd generators belongs the $2 r+1$ dimensional vector representation and has $r$ positive roots

$$
\text { short roots } \boldsymbol{\beta}_{s} \text {. }
$$

The contribution to the superpotential (3.20) from even long generators of $\mathcal{G}_{0}$ is

$$
\sum_{\boldsymbol{\beta}_{l}} \wp\left(\boldsymbol{\beta}_{l} \cdot x ; 2 \pi i, 2 \pi i \tau\right) .
$$

The contributions from the even and odd short generators are

$$
2 \sum_{\boldsymbol{\beta}_{s}}\left\{\wp\left(2 \boldsymbol{\beta}_{s} \cdot \boldsymbol{X} ; 2 \pi i, 4 \pi i \tau\right)+\wp\left(2 \boldsymbol{\beta}_{s} \cdot \boldsymbol{X}+2 \pi i \tau ; 2 \pi i, 4 \pi i \tau\right)\right\}=2 \sum_{\boldsymbol{\beta}_{s}} \wp\left(2 \boldsymbol{\beta}_{s} \cdot \boldsymbol{X}\right)
$$

by the half-period formula up to the $\tau$ only function. Together the superpotential for the $D_{r+1}^{(2)}$ case is

$$
\mathcal{W}_{D_{r+1}^{(2)}}=\sum_{\boldsymbol{\beta}_{l}} \wp\left(\boldsymbol{\beta}_{l} \cdot \boldsymbol{X}\right)+2 \sum_{\boldsymbol{\beta}_{s}} \wp\left(2 \boldsymbol{\beta}_{s} \cdot \boldsymbol{X}\right)
$$

which is manifestly $S L(2, Z)$ invariant modulo weight two. This potential is obviously associated with the untwisted elliptic Calogero-Moser system for the Lie algebra $C_{r}$ whose extended Dynkin diagram is identical to that of the coroots of $D_{r+1}^{(2)}$. 


\section{$5.5 E_{6}^{(2)}$ and $D_{4}^{(3)}$ Cases}

Let us consider the $E_{6}^{(2)}$ case first. For the $E_{6}^{(2)}$ of dimension 78 , the Lie algebra of even generators are identical to $\mathcal{G}_{0}=F_{4}$ of dimension 52 . The odd part $\mathcal{G}_{1}$ is of 26 dimensional representation of $F_{4}$. In terms of four orthonormal vectors $\boldsymbol{e}_{a}$ with $a=1,2,3,4$ the positive root vectors for even part $\mathcal{G}_{0}=F_{4}$ of $E_{6}$ are given as

$$
\text { long roots } \boldsymbol{\beta}_{l}=\boldsymbol{e}_{a} \pm \boldsymbol{e}_{b}(a<b) \text {, short roots } \boldsymbol{\beta}_{s}=\boldsymbol{e}_{a} \text {, or } \frac{1}{2}\left(\boldsymbol{e}_{1} \pm \boldsymbol{e}_{2} \pm \boldsymbol{e}_{3} \pm \boldsymbol{e}_{4}\right) \text {. }
$$

The positive root vectors for odd part $\mathcal{G}_{1}$ of $E_{6}^{(2)}$ are given as

$$
\text { short } \operatorname{roots} \boldsymbol{\beta}_{s} \text {. }
$$

Thus the even long root contribution to the superpotential (3.20) is

$$
\sum_{\boldsymbol{\beta}_{l}} \wp\left(\boldsymbol{\beta}_{l} \cdot \boldsymbol{X} ; 2 \pi i, 2 \pi i \tau\right)
$$

The sum of even and odd short root contributions is

$$
2 \sum_{\boldsymbol{\beta}_{s}}\left\{\wp\left(2 \boldsymbol{\beta}_{s} \cdot \boldsymbol{X} ; 2 \pi i, 4 \pi i \tau\right)+\wp\left(2 \boldsymbol{\beta}_{s} \cdot \boldsymbol{X}+2 \pi i \tau ; 2 \pi i, 4 \pi i \tau\right)\right\}=2 \sum_{\boldsymbol{\beta}_{s}} \wp\left(2 \boldsymbol{\beta}_{s} \cdot \boldsymbol{X}\right)
$$

again up to a $\tau$-only function by the half-period formula. Putting them together we have the effective superpotential

$$
\mathcal{W}_{E_{6}^{(2)}}=\sum_{\boldsymbol{\beta}_{l}} \wp\left(\boldsymbol{\beta}_{l} \cdot \boldsymbol{X}\right)+2 \sum_{\beta_{s}} \wp\left(2 \boldsymbol{\beta}_{s} \cdot \boldsymbol{X}\right)
$$

which has the manifest $S L(2, Z)$ property. In addition this potential is associated with the untwisted elliptic Calogero-Moser potential for the Lie group $F_{4}$, whose extended Dynkin diagram is dual to that of $E_{6}^{(2)}$.

For $D_{4}^{(3)}$ of dimension 28 , the $\mathcal{G}_{0}=G_{2}$ of even generators has 6 positive roots,

$$
\begin{aligned}
& \text { long } \operatorname{roots} \boldsymbol{\beta}_{l}=\sqrt{2} \boldsymbol{e}_{1}, \pm \sqrt{\frac{1}{2}} \boldsymbol{e}_{1}+\sqrt{\frac{3}{2}} \boldsymbol{e}_{2}, \\
& \text { short } \operatorname{roots} \boldsymbol{\beta}_{s}=\sqrt{\frac{2}{3}} \boldsymbol{e}_{2}, \pm \sqrt{\frac{1}{2}} \boldsymbol{e}_{1}+\sqrt{\frac{1}{6}} \boldsymbol{e}_{2},
\end{aligned}
$$

The $\mathcal{G}_{1}$ and $\mathcal{G}_{2}$ have each three short positive roots, which are identical to the short roots of $\mathcal{G}_{0}$.

The contribution to the superpotential (3.20) from the even long roots is

$$
\sum_{\boldsymbol{\beta}_{l}} \wp\left(\boldsymbol{\beta}_{l} \cdot \boldsymbol{X} ; 2 \pi i, 2 \pi i \tau\right)
$$


which is modular. The contribution from the even and twisted short roots of $\mathcal{G}_{n}$ with $n=0,1,2$ is

$$
3 \sum_{\boldsymbol{\beta}_{s}} \sum_{n=0}^{2}\left\{\wp\left(3 \boldsymbol{\beta}_{s} \cdot \boldsymbol{X}+2 \pi i \tau n ; 2 \pi i, 6 \pi i \tau\right)\right\}=3 \sum_{s} \wp\left(3 \boldsymbol{\beta}_{s} \cdot \boldsymbol{X} ; 2 \pi i, 2 \pi i \tau\right),
$$

up to a $\tau$ only function due to the triple period formula. The total superpotential is then

$$
\mathcal{W}_{D_{4}^{(3)}}=\sum_{\boldsymbol{\beta}_{l}} \wp\left(\boldsymbol{\beta}_{l} \cdot \boldsymbol{X}\right)+3 \sum_{\boldsymbol{\beta}_{s}} \wp\left(3 \boldsymbol{\beta}_{s} \cdot \boldsymbol{X}\right)
$$

where the sum is for all positive long and short roots of $\mathcal{G}_{n}$ of $D_{4}^{(3)}$. The result is again modular with weight two. It is also associated with the untwisted Calogero-Moser potential for the Lie algebra $G_{2}$ whose extended Dynkin diagram is dual to that of $D_{4}^{(3)}$.

\section{Glueball Superpotential}

We have found the $S L(2, Z)$ modular superpotentials for all $\mathcal{N}=1^{*}$ theory with twisted boundary condition. The superpotentials we wrote are functions of the chiral superfields, X, which are essentially the photon supermultiplet that remains unbroken by the Wilson line symmetry breaking. In turn this superpotential should lead to the gluino condensation, determining the vacuum structure and its accompanying value of the gluino condensate, among many other physical quantities.

Let us first recall how this happens. The superpotential is expected to have serveral discrete stationary points $\left\{\boldsymbol{X}_{0}\right\}$ in the fundamental domain, where

$$
\frac{\partial \mathcal{W}}{\partial X_{a}}=0, a=1, . ., r^{\prime}
$$

The number of supersymmetric ground states is the number of such stationary points modulo the Weyl reflections. The values of the superpotential at these stationary points would be holomorphic functions of only $m$ and $\tau$ variables. The derivative of the superpotential with respect to $\tau$ would lead to the expectation value of the glueball superfield $S$,

$$
\langle S\rangle=\frac{1}{2 \pi i} \frac{\partial \mathcal{W}\left(\boldsymbol{X}_{0}\right)}{\partial \tau},
$$

at a given vacuum $\boldsymbol{X}=\boldsymbol{X}_{0}$. This relation can be seen from the fact that the bare Lagrangian contains the term

$$
\left.2 \pi i \tau S\right|_{\theta^{2}}+\text { complex conjugate }
$$


which is nothing but the kinetic term for the gauge field.

This can be made more precise as follows. We will show how this goes for our (twisted) $N=1^{*}$ theories, but similar constructions are possible in other cases also. With the $\mathcal{G}_{0}$ of the rank $r^{\prime}$, the twisted affine algebra $\mathcal{G}^{(L)}$ has $r^{\prime}+1$ fundamental monopoles corresponding to the simple roots $\boldsymbol{\beta}_{a}, a=0,1,2 \ldots r^{\prime}$ of the affine algebra. We define $r^{\prime}$ independent chiral superfields

$$
Y_{a}=\boldsymbol{\beta}_{a}^{*} \cdot \boldsymbol{X},
$$

plus one more variable

$$
Y_{0}=\frac{4 \pi i \tau}{L \boldsymbol{\beta}_{0}^{2}}+\boldsymbol{\beta}_{0}^{*} \cdot \boldsymbol{X} .
$$

Due to the properties of the simple roots discussed in Appendix E, we have the constraint

$$
\sum_{a=0}^{r^{\prime}} \tilde{k}_{a}^{*} Y_{a}=\frac{4 \pi i \tau \tilde{k}_{0}^{*}}{L \boldsymbol{\beta}_{0}^{2}},
$$

with comarks $\tilde{k}_{a}^{*}$ for $\mathcal{G}^{(L)}$, which, according to (E.4), is

$$
\sum_{a=0}^{r^{\prime}} \tilde{k}_{a}^{*} Y_{a}=\frac{4 \pi i \tau}{\boldsymbol{\alpha}_{0}^{2}}=2 \pi i \tau,
$$

as the lowest root $\boldsymbol{\alpha}_{0}$ of $\mathcal{G}$ has the length square two in our convention. In terms of their exponents,

$$
y_{a}=e^{Y_{a}}, a=0,1, \ldots r^{\prime},
$$

the above constraint becomes

$$
\prod_{a=0}^{r^{\prime}} y_{a}^{\tilde{k}_{a}^{*}}=q
$$

with $q=e^{2 \pi i \tau}$ as discussed in subsection (3.3).

In small coupling constant $e^{2}$ limit, which is equivalent to large $-\operatorname{Re}(2 \pi i \tau)$, or small $|q|$ limit, we are expanding the superpotential with a chiral superfield lying near the stationary points of the superpotential (3.14), which we will see later that

$$
\boldsymbol{X} \approx 2 \pi i \tau \frac{\boldsymbol{\rho}}{h}+\delta \boldsymbol{X},
$$

with order one quantity $\delta \boldsymbol{X} . \boldsymbol{\rho}$ is the Weyl vector of the twisted algebra

$$
\boldsymbol{\rho}=\sum_{a=1}^{r^{\prime}} \boldsymbol{w}_{a}
$$


with the fundamental weights $\boldsymbol{w}_{a}$ such that $\boldsymbol{w}_{a} \cdot \boldsymbol{\beta}_{b}^{*}=\delta_{a b}$. Around this background, we see that

$$
y_{a} \sim q^{1 / h}, a=0,1,2, \ldots, r^{\prime}
$$

where for the estimation of $y_{0}$ we have used the properties of the roots as discussed in Appendices D and E.

Now, we are interested in expressing our potential in series as a sum over all possible magnetic monopole instantons and pure instanton corrections. One can make a general statement about all twisted affine algebra with a minor modification for $A_{2 r}^{(2)}$ case. In all other cases where $\beta_{0}$ is a short root with $\tilde{k}_{0}^{*}=1$, a positive co-root $\boldsymbol{\beta}^{*}$ in any level $\mathcal{G}_{n}$ can be expressible as a nonnegative integer sum of the simple co-root $\boldsymbol{\beta}_{a}^{*}$ of $\mathcal{G}_{0}$,

$$
\boldsymbol{\beta}^{*}=\sum_{a=1}^{r^{\prime}} n_{a} \boldsymbol{\beta}_{a}^{*}, \quad n_{a} \text { non negative integer }
$$

and $\sum_{a}^{r^{\prime}} n_{a} \leq h-1$, where the inequality is saturated when (6.13) becomes $-\boldsymbol{\beta}_{0}^{*}$. The superpotential is a sum of Weierstrass functions whose arguments are $\boldsymbol{\beta}^{*} \cdot \boldsymbol{X}$. Take any one of these functions and expand it in small $q$, and we find some general series like

$$
\wp\left(\boldsymbol{\beta}^{*} \cdot \boldsymbol{X}\right)=\frac{1}{12}+\sum_{k=0}^{\infty} k y^{k}+\sum_{k=1}^{\infty} \sum_{n=1}^{\infty} k q^{k n}\left(y^{k}+y^{-k}-2\right),
$$

where $y=e^{\boldsymbol{\beta}^{*} \cdot \boldsymbol{X}}$. From the $\boldsymbol{\beta}^{*}$ decomposition $(6.13)$ in terms of simple roots, we also have

$$
y=\prod_{a=1}^{r^{\prime}} y_{a}^{n_{a}}
$$

As we have $q$ expressed in terms of $y_{a}$ as in Eq.(6.9), and noting that $q y^{-1}$ can always be expressed in positive powers in $y_{a}$ 's, we can see the superpotential as an infinite sum over multi-monopole-instanton contributions

$$
\mathcal{W}=m^{3} \sum_{K=\left\{n_{a}\right\}} c_{K} \prod_{a=0}^{r^{\prime}} y_{a}^{n_{a}}
$$

where the sum is for all possible sets $K=\left\{n_{a}\right\}$ of non negative integers $n_{a}$ and the coefficients $c_{K}$ are determined by our $S L(2, Z)$ invariant superpotential. For the $A_{2 r}^{(2)}$ case, whose Weierstrass function involves shifted arguments, we will also see later that all corrections can be expressed as the products of the contributions from the fundamental monopoles. 
This expression is not entirely correct since not all of $Y_{a}^{\prime}$ 's are dynamical variables. In order to have a sensible superpotential we must introduce a Lagrange multiplier field $S$ as follows

$$
\mathcal{W}\left(Y_{a}, S ; \tau\right)=m^{3} \sum_{K=\left\{n_{a}\right\}} c_{K} \prod_{a=0}^{r^{\prime}} y_{a}^{n_{a}}-S\left(-2 \pi i \tau+\sum_{a=0}^{r^{\prime}} \tilde{k}_{a}^{*} Y_{a}\right)
$$

which removes one variable $\sum_{a=0}^{r^{\prime}} \tilde{k}_{a}^{*} Y_{a}$ and replace it by one parameter $\tau$. It is now quite obvious that $S$ is nothing but the glueball superfield, and the gluino condensate is given by

$$
\langle S\rangle \sim \frac{\partial \mathcal{W}}{\partial \tau}
$$

from the equation of motion for $S$. It is then a matter of integrating out the superfields $Y_{a}$ to obtain the glueball superpotential.

$$
\mathcal{W}(S ; \tau)=\left.\mathcal{W}\left(Y_{a}, S ; \tau\right)\right|_{\text {extremize w.r.t. } Y_{a}^{\prime} \text { 's }}
$$

This sort of duality or mirror symmetry between two types of superpotential has appeared first in Ref. 28] and has been used extensively in a recent work 29].

In this section, we will show that this glueball superpotential for $\mathcal{N}=1^{*}$ theory with twisted boundary condition matches exactly with that for the $\mathcal{N}=1^{*}$ theory with periodic boundary condition in leading terms in the series expansion. This might sound a bit odd initially, given that we are dealing with two theories of completely different gauge groups and matter content, for example in the small radius limit. The first subsection will motivate this identity and explain why this must be the case.

In particular, when the dual Coxeter number $h$ is small enough, the pure instanton correction of order $q$ is included in this comparison. The pure instanton correction for the twisted case is fixed by the $S L(2, Z)$ symmetry, so this comparison of two glueball superpotentials may be regarded as an independent check of our prescription.

\section{$6.1 \mathcal{W}^{V Y}$ and Universality}

In the leading order (or to order $q^{\frac{1}{h}}$ ) we keep only terms linear in $y_{a}$, or the corrections from the fundamental monopoles. The superpotential (3.14) in the $\mathcal{N}=1$ theory of the twisted affine algebra $\mathcal{G}^{(L)}$ can be rewritten as

$$
\mathcal{W}_{N=1}\left(Y_{a} ; S\right)=m^{3}\left(\sum_{a=0}^{r^{\prime}} \frac{2}{\boldsymbol{\beta}_{a}^{2}} y_{a}\right)-S\left(-2 \pi i \tau+\sum_{a=0}^{r^{\prime}} \tilde{k}_{a}^{*} Y_{a}\right)
$$


where $\tilde{k}_{a}^{*}$ is the comarks of the twisted Lie algebra. Integrating over the glueball field $S$ renders $Y_{0}$ to be a linear combination of other $Y^{\prime}$ 's, leading to the Toda potential (3.14).

Integrating over $Y_{a}$ instead leads to a glueball superpotential

$$
\mathcal{W}(S ; \tau)=h S-S \ln \left(\frac{S}{\Lambda^{3}}\right)^{h}
$$

where $h$ is the dual Coxeter number of $\mathcal{G}$ and the confinement scale in the Coulomb phase is now given as

$$
\Lambda^{3}=m^{3} q^{\frac{1}{h}} \prod_{a=0}^{r^{\prime}}\left[\frac{\tilde{k}_{a}^{*} \boldsymbol{\beta}_{a}^{2}}{2}\right]^{\tilde{k}_{a}^{*}} .
$$

The stationary point of the above superpotential leads to the glueball field expectation value (2.9) in $h$ number of vacua. For a given vacuum, the expectation value of $Y_{a}$ or $y_{a}$ can be read directly from the above action $(6.20)$, which are

$$
\left\langle Y_{a}\right\rangle=\frac{2 \pi i k}{h}+\ln \left(\frac{\tilde{k}_{a}^{*} \boldsymbol{\beta}_{a}^{2} \Lambda^{3}}{2 m^{3}}\right), k=0,1, \ldots,(h-1),
$$

and so $\left\langle y_{a}\right\rangle \sim q^{1 / h}$, which is consistent. Indeed these stationary points are consistent with the assumption for the stationary points (6.10) of the chiral superfield $\boldsymbol{X}$. The weak coupling series of the glueball superpotential leads to the corrections to these stationary points by a similar series in powers of $q^{1 / h}$.

Note that the form of the superpotential of the twisted theory $\mathcal{G}^{(L)}$ is the same as that of the untwisted theory $\mathcal{G}$, namely the Veneziano-Yankielowicz superpotential. Furthermore, the identities in Appendix E shows that the confinement scale of the $\mathcal{N}=1$ theory with twisted boundary condition is identical to that of the $\mathcal{N}=1$ theory with periodic boundary condition, resulting in the identical glueball potential.

We cannot possibly say the same thing about the superpotential $\mathcal{W}(\boldsymbol{X})$. To begin with, the untwisted $\mathcal{G}$ theory and the twisted $\mathcal{G}^{(L)}(L>1)$ theory have different ranks, and therefore their superpotentials are analytic functions of different number of complex variables. Thus, it must be quite surprising to see that one and the same glueball superpotential is found when we started with two such different superpotential.

A hint of why this happens can be found in the fact that superpotential $\mathcal{W}(\boldsymbol{X})$ has no explicit dependence on the compactification radius. If we had not known better, this might lead us to conclude that the superpotential is independent of the twist, the argument being that a large radius limit can always wash out any boundary 
condition. However, this is not the case with $\mathcal{W}(\boldsymbol{X})$ as we just explained. ${ }^{5} \mathcal{W}(\boldsymbol{X})$ of the twisted theory do retain the memory of the twisting in that it is defined with respect to Cartan sector of $\mathcal{G}_{0}$ instead of entire $\mathcal{G}$.

The glueball superpotential, however, is defined as over a single chiral field, $S$, whose definition involves tracing over all gauge indices. This means that $\mathcal{W}(S ; \tau)$ does not retain the memory of the twist in the way $\mathcal{W}(\boldsymbol{X})$ does, and is really insensitive to the boundary condition in the large radius limit. Unlike $\mathcal{W}(\boldsymbol{X})$, then, the glueball superpotential must be insensitive to the twist at all radius, which explains why we find the identical Veneziano-Yankielowicz superpotential $\mathcal{W}^{V Y}$ for the twisted and the untwisted cases alike. We expect this exact matching of glueball superpotentials of twisted and untwisted theory to persist to all orders beyond $\mathcal{W}^{V Y}$. The rest of this section is devoted to checking this to the 4 th power in $S$.

\subsection{Glueball Superpotential of Untwisted $A D E$ Theories}

Let us compute the glueball superpotential of untwisted theories with simply laced groups, $A_{r}, D_{r}$ and $E_{6}, E_{7}, E_{8}$ to order $S^{4}$. As we noted already, all twisted theories arise from one of these simply laced cases, and in the following subsections we will compare the results here with their twisted counterpart.

It is convenient to introduce the extended Cartan matrix $\hat{C}_{a b}$ and the symmetric incidence matrix $\mathcal{I}_{a b}$ such that $\hat{C}_{a b}=2 \boldsymbol{\alpha}_{a} \cdot \boldsymbol{\alpha}_{b}^{*}=2 \delta_{a b}-\mathcal{I}_{a b}$, where $a, b=0, \ldots, r$. The incidence matrix $\mathcal{I}_{a b}$ is zero (one) when two simple roots $\boldsymbol{\alpha}_{a}$ and $\boldsymbol{\alpha}_{b}$ are disconnected (connected) in the extended Dynkin diagram. The diagonal elements of the incidence matrix vanish. The simple roots are $\boldsymbol{\alpha}_{a}$ with $a=0,1,2 \ldots r$, where $r$ is the rank of the algebra. The dual Coxeter number is $h$ and we define $Y_{0}=2 \pi i \tau-\boldsymbol{\alpha}_{0} \cdot \boldsymbol{X}$ and $Y_{a}=\boldsymbol{\alpha}_{a} \cdot \boldsymbol{X}, a=1,2 \ldots r$.

The superpotential is

$$
\mathcal{W}=\sum_{\boldsymbol{\alpha}>0} \wp\left(\boldsymbol{\alpha}^{*} \cdot x\right)
$$

where the sum is over the positive roots of the respective $A D E$ algebra. This may be expanded in a series of the contributions to the fundamental monopole instantons with $y_{a}=e^{Y_{a}}$ as above. Keeping terms up to order $q^{\frac{4}{h}}$, we find that the series

\footnotetext{
${ }^{5}$ This observation seems at odd with the notion that at least in the larger radius limit the physics must be independent of boundary condition. What must be happening here is that $\mathcal{W}(\boldsymbol{X})$ of the twisted theory is the result of integrating out more degrees of freedom than $\mathcal{W}(\boldsymbol{X})$ of the untwisted theories, and in the large radius limit, the former is less reliable as a low energy superpotential.
} 
becomes $^{6}$

$$
\begin{aligned}
\mathcal{W}_{A^{(1)}, D^{(1)}, E^{(1)}=} & \sum_{a=0}^{r}\left(y_{a}+2 y_{a}^{2}+3 y_{a}^{3}+4 y_{a}^{4}\right)+\frac{1}{2} \sum_{a b} \mathcal{I}_{a b} y_{a} y_{b}+\frac{1}{2} \sum_{a b c} I_{a b} I_{b c} y_{a} y_{b} y_{c} \\
& -\frac{1}{2} \sum_{a b} I_{a b} y_{a}^{2} y_{b}+\frac{1}{2} \sum_{a b c d} I_{a b} I_{b c} I_{c d} y_{a} y_{b} y_{c} y_{d}-\sum_{a b c} I_{a b} I_{a c} y_{a}^{2} y_{b} y_{c} \\
& +\frac{3}{2} \sum_{a b} I_{a b} y_{a}^{2} y_{b}^{2}+\frac{1}{6} \sum_{a b c d} I_{a b} I_{a c} I_{a d} y_{a} y_{b} y_{c} y_{d}-\frac{1}{2} \sum_{a b c} I_{a b} I_{a c} y_{a} y_{b}^{2} y_{c} \\
& +\frac{1}{3} \sum_{a b} I_{a b} y_{a} y_{b}^{3}-S\left(-2 \pi i \tau+\sum_{a=0}^{r} k_{a}^{*} Y_{a}\right),
\end{aligned}
$$

where $k_{a}^{*}$ is the comarks of the untwisted affine algebra. After integration over $Y_{a}$, we obtain the superpotential for the glueball superpotential to order $S^{4}$,

$$
\begin{aligned}
\mathcal{W}_{A^{(1)}, D^{(1)}, E^{(1)}}(S ; \tau) & =\mathcal{W}_{A, D, E}^{V Y} \\
& +3 h_{2} S^{2}-14 h_{3} S^{3}+\left(115 h_{4}-\frac{23}{2} \hat{C}_{a b}\left(k_{a}^{*}\right)^{2}\left(k_{b}^{*}\right)^{2}\right) S^{4} \\
& +\mathcal{O}\left(S^{5}\right)
\end{aligned}
$$

where $h_{n}=\sum_{a=0}^{r}\left(k_{a}^{*}\right)^{n}$ with the comarks $k_{a}^{*}$.

Note that for all simply laced groups, $\hat{C}_{a b} k_{b}^{*}=0$. For $A_{r}, k_{a}^{*}=1$ for all $a=$ $0,1, \ldots, r$, and so the last term is absent for the theory with $A_{r}$. This last term proportional to $C_{a b}\left(k_{a}^{*}\right)^{2}\left(k_{b}^{*}\right)^{2}$ is nontrivial for the other, $D$ and $E$ type, simply laced algebra. This particular term appears missing in the result of Ref. [29.

\section{$6.3 A_{r}^{(1)}$ vs. $A_{r}^{(2)}$}

Let us write out the case of $A_{r}$ series more explicitly here. For untwisted $A_{r}$ theory, the simple roots are

$$
\boldsymbol{\alpha}_{a}=\boldsymbol{e}_{a}-\boldsymbol{e}_{a+1}, a=0,1, \ldots, r
$$

where $\boldsymbol{e}_{r+1}=\boldsymbol{e}_{0}$. With $Y_{0}=2 \pi i \tau-\boldsymbol{\alpha}_{0} \cdot \boldsymbol{X}$ and $Y_{a}=\boldsymbol{\alpha}_{a} \cdot \boldsymbol{X}, a=1,2, \ldots r$, and $y_{a}=e^{Y_{a}}$, we can expand the above superpotential in a series, to order $q^{8 / h}$. Writing

\footnotetext{
${ }^{6}$ For the rest of this section, we drop the mass parameter $m^{3}$ for simplicity.
} 
out the above expansion of the superpotential for $A_{r}$ untwisted theory, we find

$$
\begin{aligned}
\mathcal{W}_{A_{r}^{(1)}}= & \sum_{a=0}^{r}\left(y_{a}+2 y_{a}^{2}+3 y_{a}^{3}+4 y_{a}^{4}+5 y_{a}^{5}+6 y_{a}^{6}+7 y_{a}^{7}+8 y_{a}^{8}+y_{a} y_{a+1}+2 y_{a}^{2} y_{a+1}^{2}\right. \\
& +3 y_{a}^{3} y_{a+1}^{3}+4 y_{a}^{4} y_{a+1}^{4}+y_{a} y_{a+1} y_{a+2}+2 y_{a}^{2} y_{a+1}^{2} y_{a+2}^{2}+y_{a} y_{a+1} y_{a+2} y_{a+3} \\
& +2 y_{a}^{2} y_{a+1}^{2} y_{a+2}^{2} y_{a+3}^{2}+y_{a} y_{a+1} y_{a+2} y_{a+3} y_{a+4}+y_{a} y_{a+1} y_{a+2} y_{a+3} y_{a+4} y_{a+5} \\
& +y_{a} y_{a+1} y_{a+2} y_{a+3} y_{a+4} y_{a+5} y_{a+6} y_{a} y_{a+1} y_{a+2} y_{a+3} y_{a+4} y_{a+5} y_{a+6} y_{a+7} \\
& \left.+y_{a} y_{a+1} y_{a+2} y_{a+3} y_{a+4} y_{a+5} y_{a+6} y_{a+7} y_{a+8}\right)-S\left(-2 \pi i \tau+\sum_{a=0}^{r} Y_{a}\right) .
\end{aligned}
$$

Here we put the periodic condition on the indices so that $y_{r+1}=y_{0}$ and so on. Solving the equation for $Y_{a}$, we get the glueball superpotential in a series,

$$
\begin{aligned}
\mathcal{W}_{A_{r}^{(1)}}(S ; \tau)= & \mathcal{W}_{A_{r}}^{V Y}+h\left(3 S^{2}-14 S^{3}+115 S^{4}-1206 S^{5}\right. \\
& \left.+\frac{72576}{5} S^{6}-190968 S^{7}+\frac{18721233}{7} S^{8}\right)+\mathcal{O}\left(S^{9}\right)
\end{aligned}
$$

This particular series is valid up to 8-th power in $S$ for the cases with dual Coxeter number $h=r+1$ larger than 8 .

Now let us turn to the twisted cases. First, take the case of $A_{r=2 r^{\prime}}^{(2)}$, whose zero degree subgroup is $\mathcal{G}_{0}=S p\left(r^{\prime}\right)=U S p\left(2 r^{\prime}\right)$ of rank $r^{\prime}$ and the dual Coxeter number is $h=2 r^{\prime}+1=r+1$. For $A_{2 r^{\prime}}^{(2)}$, the root vectors given in orthonomal vectors were studied in the subsection (4.2). The simple roots, dropping the degree, are

$$
\boldsymbol{\beta}_{0}=-\sqrt{2} \boldsymbol{e}_{1}, \quad \boldsymbol{\beta}_{a}=\frac{\boldsymbol{e}_{a}-\boldsymbol{e}_{a+1}}{\sqrt{2}}, a=1,2, \ldots\left(r^{\prime}-1\right), \quad \boldsymbol{\beta}_{r^{\prime}}=\frac{\boldsymbol{e}_{r^{\prime}}}{\sqrt{2}}
$$

with $\boldsymbol{e}_{a}$ with $a=1, \ldots, r^{\prime}$ being orthonormal vectors.

The superpotential (5.20) can be expanded in small $q$ series. With $Y_{0}=\pi i \tau+$ $\boldsymbol{\beta}_{0} \cdot \boldsymbol{X}, Y_{a}=\boldsymbol{\beta}_{a} \cdot \tau$ with $a=1,2, \ldots r$, all variables $y_{a}=e^{Y_{a}} \sim q^{\frac{1}{h}}$. For a medium size root $\boldsymbol{\beta}_{m}$, we get the series expansion. For the long positive root $\boldsymbol{\beta}_{l}$ contribution $\wp\left(\boldsymbol{\beta}_{l} \cdot \boldsymbol{X}-\pi i \tau\right)$, we notice that

$$
\pi i \tau-\boldsymbol{\beta}_{l} \cdot \boldsymbol{X}=Y_{0}+\sum_{a=1}^{r^{\prime}} n_{a} Y_{a}
$$

where $n_{a}$ are non negative integers. The reason is that a long negative root $-\boldsymbol{\beta}_{l}$ can be expressed as a sum of the lowest long negative root $\boldsymbol{\beta}_{0}$ plus simple roots. Thus the series will be an infinite series of the contributions from the fundamental monopoles. 
For the rest two contributions for a long positive root, we employ the identity

$$
\begin{aligned}
& \wp\left(\boldsymbol{\beta}_{l} \cdot \boldsymbol{X}\right)+\wp\left(\boldsymbol{\beta}_{l} \cdot \boldsymbol{X}+\pi i\right) \\
& =\frac{2}{12} \sum_{k=1}^{\infty}\left(1+(-1)^{k}\right) k e^{\boldsymbol{\beta}_{l} \cdot \boldsymbol{X}}+\sum_{k, m=1}^{\infty} k q^{k m}\left(\left(1+(-1)^{k}\right)\left(e^{\boldsymbol{\beta}_{l} \cdot \boldsymbol{X}}+e^{-\boldsymbol{\beta}_{l} \cdot \boldsymbol{X}}\right)-4\right) \\
& =\frac{2}{12}+4 \sum_{k} k e^{\boldsymbol{\beta}_{s}^{*} \cdot \boldsymbol{X}}+4 \sum_{k, m=1}^{\infty} k q^{2 k m}\left(e^{\boldsymbol{\beta}_{s}^{*} \cdot \boldsymbol{X} k}+e^{-\boldsymbol{\beta}_{s}^{*} \cdot \boldsymbol{X}}\right)-4 \sum_{k, m=1}^{\infty} k q^{k m},
\end{aligned}
$$

where $\boldsymbol{\beta}_{s}^{*}=2 \boldsymbol{\beta}_{l}$. Since $q$ is a product of $y_{a}$ 's and $q^{2} e^{-\boldsymbol{\beta}_{s}^{*} \cdot \boldsymbol{X}}$ can be expressed in the positive power products of $y_{a}$, the above contributions can be expressed in a series of contributions from the fundamental monopoles. The factor four is exactly the needed coefficient $2 / \boldsymbol{\beta}_{s}^{2}$.

Once that is done, we can find the superpotential in the series expansion to order $q^{\frac{4}{h}}$

$$
\begin{aligned}
\mathcal{W}_{A_{2 r^{\prime}}^{(2)}=} & \left(y_{0}+2 y_{0}^{2}+3 y_{0}^{3}+4 y_{0}^{4}\right)+2 \sum_{a=1}^{r^{\prime}-1}\left(y_{a}+2 y_{a}^{2}+3 y_{a}^{3}+4 y_{a}^{4}\right) \\
& +4\left(y_{r^{\prime}}+2 y_{r^{\prime}}^{2}+3 y_{r^{\prime}}^{3}+4 y_{r^{\prime}}^{4}\right)+\left(y_{0} y_{1}+2 y_{0}^{2} y_{1}^{2}\right)+2 \sum_{a=1}^{r^{\prime}-1}\left(y_{a} y_{a+1}+2 y_{a}^{2} y_{a+1}^{2}\right) \\
& +4 y_{r^{\prime}-1}^{2} y_{r^{\prime}}+y_{0} y_{1} y_{2}+2 y_{0}^{2} y_{1}+2 \sum_{a=1}^{r^{\prime}-2} y_{a} y_{a+1} y_{a+2} \\
& +y_{0} y_{1} y_{2} y_{3}+2 y_{0}^{2} y_{1} y_{2}+2 y_{r^{\prime}-1} y_{r^{\prime}-1}^{2} y_{r^{\prime}}+2 \sum_{a=1}^{r^{\prime}-3} y_{a} y_{a+1} y_{a+2} y_{a+3} \\
& -S\left(-2 \pi i \tau+2 Y_{0}+2 Y_{1}+\cdots+2 Y_{r^{\prime}-1}+Y_{r^{\prime}}\right) .
\end{aligned}
$$

After integrating over $Y_{a}$ order by order in perturbation, we obtain the glueball superpotential

$$
\mathcal{W}_{A_{2 r^{\prime}}^{(2)}}(S ; \tau)=\mathcal{W}_{A_{2 r^{\prime}}}^{V Y}+h\left(3 S^{2}-14 S^{3}+115 S^{4}\right)+\mathcal{O}\left(S^{5}\right)
$$

To the order $S^{4}$, this series matches exactly with the series expansion obtained for the theory with untwisted affine $A_{r=2 r^{\prime}}^{(1)}$ algebra above. The contributions from the constant coefficient 1/12 matches exactly between the twisted and untwisted glueball superpotential due to the fact (E.6) of Appendix E. Such match of constants occurs also for other twisted cases due to the fact (E.7).

The second case to consider is $A_{r=2 r^{\prime}-1}^{(2)}$, whose zero grading is $\mathcal{G}_{0}=C_{r^{\prime}}$ with rank $r^{\prime}$ and the dual Coxeter number $h=2 r^{\prime}=r+1$. The roots of this twisted affine 
algebra is studied in Sec. (4.3). The simple roots are

$$
\boldsymbol{\beta}_{0}=-\frac{\boldsymbol{e}_{1}+\boldsymbol{e}_{2}}{\sqrt{2}}, \quad \boldsymbol{\beta}_{a}=\frac{\boldsymbol{e}_{a}-\boldsymbol{e}_{a+1}}{\sqrt{2}}, a=1,2 \ldots\left(r^{\prime}-1\right), \quad \boldsymbol{\beta}_{r^{\prime}}=\sqrt{2} \boldsymbol{e}_{r^{\prime}} .
$$

With $Y_{0}=2 \pi \tau-\boldsymbol{\beta}_{0}^{*} \cdot \boldsymbol{X}$ and $Y_{a}=\boldsymbol{\beta}_{a}^{*} \cdot \boldsymbol{X}, a=1,2, \ldots r$, we introduce again the contributions from the fundamental monopoles, $y_{a}=e^{Y_{a}} \sim q^{\frac{1}{h}}$. The contributions from the short and long roots to the superpotential (5.25) can be now expressed in the series of $y_{a}$. Expanding the superpotential (5.25) to order $q^{\frac{4}{h}}$, we obtain the series,

$$
\begin{aligned}
\mathcal{W}_{A_{2 r^{\prime}-1}^{(2)}}= & 2 \sum_{a=0}^{r^{\prime}-1}\left(y_{a}+2 y_{a}^{2}+3 y_{a}^{3}+4 y_{a}^{4}\right)+\left(y_{r^{\prime}}+2 y_{r^{\prime}}^{2}+3 y_{3}^{3}+4 y_{r^{\prime}}^{4}\right)+2\left(y_{0} y_{2}+2 y_{0}^{2} y_{2}^{2}\right) \\
& +2 \sum_{a=1}^{r^{\prime}-2}\left(y_{a} y_{a+1}+2 y_{a}^{2} y_{a+1}^{2}\right)+\left(y_{r^{\prime}-1} y_{r^{\prime}}+2 y_{r^{\prime}-1}^{2} y_{r^{\prime}}^{2}\right)+2 y_{0} y_{2} y_{3} \\
& +2 \sum_{a=0}^{r^{\prime}-3}\left(y_{a} y_{a+1} y_{a+2}\right)+y_{r^{\prime}-2} y_{r^{\prime}-1} y_{r^{\prime}}+2 y_{r^{\prime}-1} y_{r^{\prime}}^{2}+2 y_{0} y_{2} y_{3} y_{4} \\
& +2 \sum_{a=0}^{r^{\prime}-4}\left(y_{a} y_{a+1} y_{a+2} y_{a+3}\right)+y_{r^{\prime}-3} y_{r^{\prime}-2} y_{r^{\prime}-1} y_{r^{\prime}}+2 y_{r^{\prime}-2} y_{r^{\prime}-1} y_{r^{\prime}}^{2} \\
& -S\left(-2 \pi i \tau+Y_{0}+Y_{1}+2 \sum_{a=2}^{r^{\prime}} Y_{a}\right) .
\end{aligned}
$$

After solving the equation for $Y_{a}$, and replacing the value of $Y_{a}$ in the potential, we obtain the glueball superpotential in a series to order $S^{4}$

$$
\mathcal{W}_{A_{2 r^{\prime}-1}^{(2)}}(S ; \tau)=\mathcal{W}_{A_{2 r^{\prime}-1}^{V Y}}^{V Y}+h\left(3 S^{2}-14 S^{3}+115 S^{4}\right)+\mathcal{O}\left(S^{5}\right)
$$

Again this series matches exactly with that of the theory with untwisted affine algebra $A_{r=2 r^{\prime}-1}^{(1)}$ above.

\section{$6.4 A_{2}^{(1)}$ vs. $A_{2}^{(2)}$}

In the present computation, we are comparing twisted and untwisted theories to quartic order in $S$. Because of this, the case of $A_{r}$ with $r \leq 3$ deserves special attention: the pure instanton correction that we found in previous section begins to enter at order $S^{h}$, which means that this correction is important for our comparison in the case of $A_{2}$ and $A_{3}$. To check that our proposal of exact Coulombic superpotential is correct, we must be more careful to include this effect.

The superpotential for the theory with $A_{2}=S U(3)$ and periodic boundary condition is

$$
\mathcal{W}_{A_{2}^{(1)}}=\wp\left(\boldsymbol{\alpha}_{0} \cdot \boldsymbol{X}\right)+\wp\left(\boldsymbol{\alpha}_{1} \cdot \boldsymbol{X}\right)+\wp\left(\boldsymbol{\alpha}_{2} \cdot \boldsymbol{X}\right)
$$


It is an even double periodic function of a two dimensional vector $\boldsymbol{X}$ with the proper $S L(2, Z)$ symmetry. It has five stationary points modulo Weyl symmetry, four of which are single zeros and describe massive vacua and one of which is a double zero and so describes massless vacua [20]. Note that $\boldsymbol{\alpha}_{1}$ and $\boldsymbol{\alpha}_{2}$ are simple roots and $\boldsymbol{\alpha}_{0}=-\boldsymbol{\alpha}_{1}-\boldsymbol{\alpha}_{2}$ is the lowest negative root. We introduce three variables, $Y_{1}=\boldsymbol{\alpha}_{1} \cdot \boldsymbol{X}, Y_{2}=\boldsymbol{\alpha}_{2} \cdot \boldsymbol{X}$ and $Y_{0}=2 \pi i \tau+\boldsymbol{\alpha}_{0} \cdot \boldsymbol{X}$. We assume that $y_{a} \equiv e^{Y_{a}}$ are of order $q^{1 / 3}$, and note that $q=y_{1} y_{2} y_{3}$. The series expansion of the potential to $q^{2}$ order becomes

$$
\begin{aligned}
\mathcal{W}_{A_{2}^{(1)}}= & \sum_{a=0}^{2}\left(y_{a}+2 y_{a}^{2}+3 y_{a}^{3}+4 y_{a}^{4}+5 y_{a}^{5}+6 y_{a}^{6}\right) \\
& +\sum_{a=0}^{2}\left\{y_{a} y_{a+1}+2\left(y_{a} y_{a+1}\right)^{2}+3\left(y_{a} y_{a+1}\right)^{3}\right\} \\
& +y_{0} y_{1} y_{2}\left(y_{0}+y_{1}+y_{2}\right)+y_{0} y_{1} y_{2}\left(y_{0} y_{1}+y_{1} y_{2}+y_{0} y_{1}\right) \\
& -6 y_{0} y_{1} y_{2}-18\left(y_{0} y_{1} y_{2}\right)^{2}-S\left(-2 \pi i \tau+Y_{0}+Y_{1}+Y_{2}\right) .
\end{aligned}
$$

Notice that this superpotential has also contributions from pure instantons without any magnetic charge at order $q$ and $q^{2}$.

We can integrate over $Y_{a}$ and obtain the value of $Y_{a}$ in the series expansion in $S$, which can be regarded as of order $q^{1 / 3}$. Inserting the values of $Y_{a}$ back to the superpotential, we obtain the $\mathcal{W}_{V Y}$ potential plus the corrections. The resulting glueball potential for $S U(3)$ in series to order $S^{6}$ is

$$
\mathcal{W}_{A_{2}^{(1)}}(S ; \tau)=\mathcal{W}_{A_{2}}^{V Y}+3\left(3 S^{2}-17 S^{3}+169 S^{4}-\frac{4221}{2} S^{5}+\frac{150336}{5} S^{6}\right)+\mathcal{O}\left(S^{7}\right) .
$$

Turning to the twisted case, $A_{2}^{(2)}$, our proposal for the superpotential is

$$
\mathcal{W}_{A_{2}^{(2)}}=\wp\left(\boldsymbol{\alpha}_{0} \cdot \boldsymbol{X}\right)+\wp\left(\boldsymbol{\alpha}_{0} \cdot \boldsymbol{X}+\pi i\right)+\wp\left(\boldsymbol{\alpha}_{0} \cdot \boldsymbol{X}+\pi i \tau\right),
$$

where $\boldsymbol{\alpha}_{0} \cdot \boldsymbol{X}=-\sqrt{2} X$ is defined on complex plane. One can argue that there are five gauge inequivalent vacua of this potential, whose characteristics should be identical to the periodic case with the potential (6.38). In the series expansion, we note that $\frac{2 \pi i \tau}{2}-\sqrt{2} X=Y_{0}$ and $2 \sqrt{2} X=Y_{1}$. Thus, each terms in the above potential has the series expansion as in $A_{2 r}^{(2)}$ case in subsection (6.2). Together, the twisted superpotential can be expanded to $q^{2}$ order to be

$$
\begin{aligned}
\mathcal{W}_{A_{2}^{(2)}}= & y_{0}+2 y_{0}^{2}+3 y_{0}^{3}+4 y_{0}^{4}+5 y_{0}^{5}+6 y_{0}^{6}+4\left(y_{1}+2 y_{1}^{2}+3 y_{1}^{3}+4 y_{1}^{4}+5 y_{1}^{5}+6 y_{1}^{6}\right) \\
& +y_{0} y_{1}+2\left(y_{0} y_{1}\right)^{2}+3\left(y_{0} y_{1}\right)^{3}+y_{0}^{3} y_{1}+y_{0}^{3} y_{1}^{2}+4 y_{0}^{4} y_{1}-6 y_{0}^{2} y_{1}-18 y_{0}^{4} y_{1}^{2} \\
& -S\left(-2 \pi \tau+2 Y_{0}+Y_{1}\right) .
\end{aligned}
$$


The purely instantonic contribution is $-6 y_{0}^{2} y_{1}-18\left(y_{0}^{2} y_{1}\right)^{2}$ in the above expression. After integration over $Y_{a}$, one obtains the exactly identical series (6.40) of the glueball superpotential. The $S L(2, Z)$ symmetry of the superpotential for the twisted case is crucial for this equivalence of the glueball superpotential. While we have worked out the series to order $S^{6}$, we expect that the equivalence persists to all order. It is interesting to see whether it is the case.

6.5 $D_{r}^{(1)}$ vs. $D_{r}^{(2)}$

For $D_{r=r^{\prime}+1}^{(2)}$, we have $\mathcal{G}_{0}=B_{r^{\prime}}$ whose root system is studied in Sec. $(4,4)$. The simple roots are

$$
\boldsymbol{\beta}_{0}=-\boldsymbol{e}_{1}, \quad \boldsymbol{\beta}_{a}=\boldsymbol{e}_{a}-\boldsymbol{e}_{a+1}, a=1,2, \ldots\left(r^{\prime}-1\right), \quad \boldsymbol{\beta}_{r^{\prime}}=\boldsymbol{e}_{r^{\prime}} .
$$

With the parameters $Y_{0}=2 \pi i \tau+\boldsymbol{\beta}_{0} \cdot \boldsymbol{X}$ and $Y_{a}=\boldsymbol{\beta}_{a}^{*} \cdot \boldsymbol{X}, a=1,2, \ldots r^{\prime}$ and $y_{a}=e^{Y_{a}}$, the superpotential (5.30) in series expansion becomes

$$
\begin{aligned}
\mathcal{W}_{D_{r^{\prime}+1}^{(2)}}= & 2\left(y_{0}+2 y_{0}+3 y_{0}^{3}+4 y_{0}^{4}\right) \\
& +\sum_{a=1}^{r^{\prime}-1}\left(y_{a}+2 y_{a}^{2}+3 y_{a}^{3}+4 y_{a}^{4}\right)+2\left(y_{r^{\prime}}+2 y_{r^{\prime}}^{2}+3 y_{r^{\prime}}^{3}+4 y_{r^{\prime}}^{4}\right) \\
& +\sum_{a=0}^{r^{\prime}-1}\left(y_{a} y_{a+1}+2 y_{a}^{2} y_{a+1}^{2}\right)+2 y_{0} y_{1}^{2} \\
& +\sum_{a=0}^{r^{\prime}-2} y_{a} y_{a+1} y_{a+2}+2 y_{r^{\prime}-1}^{2} y_{r^{\prime}}+y_{0} y_{1}^{2} y_{2} \\
& +\sum_{a=0}^{r^{\prime}-3}\left(y_{a} y_{a+1} y_{a+2} y_{a+3}\right)+y_{r^{\prime}-2} y_{r^{\prime}-1}^{2} y_{r^{\prime}} \\
& -S\left(-2 \pi i \tau+Y_{0}+2 \sum_{a=1}^{r^{\prime}-1} Y_{a}+Y_{r^{\prime}}\right) .
\end{aligned}
$$

Integration over $Y_{a}$ leads to the glueball superpotential in a series expansion. The series to order $S^{4}$ becomes

$$
\begin{aligned}
\mathcal{W}_{D_{r^{\prime}+1}^{(2)}}(S ; \tau) & =\mathcal{W}_{D_{r^{\prime}+1}}^{V Y}+12\left(r^{\prime}-1\right) S^{2}-56\left(2 r^{\prime}-3\right) S^{3}+368\left(5 r^{\prime}-9\right) S^{4} \\
& =\mathcal{W}_{D_{r^{\prime}+1}}^{V Y}+3 h_{2} S^{2}-14 h_{3} S^{3}+115\left(h_{4}-\frac{4}{5}\right) S^{4}+\mathcal{O}\left(S^{5}\right)
\end{aligned}
$$

where $h_{n}=\sum_{a=0}^{r^{\prime}+1}\left(k_{a}^{*}\right)^{n}$ with the comarks $k_{a}^{*}$ of the untwisted Lie algebra $D_{r^{\prime}+1}^{(1)}$. This series matches the glueball superpotential (6.26) for the untwisted $D_{r=r^{\prime}+1}^{(1)}$ case. 
As in the case of $A_{r}$ series, the above comparison is correct for large $h$ so that the pure instanton effect does not show up to order $S^{4}$. The theory with $D_{3}^{(2)}$ should be compared with the untwisted theory of gauge group $D_{3}\left(=A_{3}\right)=S U(4)$ with $h=4$. To order $S^{4}$, the glueball superpotential becomes

$$
\mathcal{W}_{D_{3}^{(2)}}(S ; \tau)=\mathcal{W}_{D_{3}}^{V Y}+4\left(3 S^{2}-14 S^{3}+\left(115-\frac{5}{2}\right) S^{4}\right)+\mathcal{O}\left(S^{5}\right)
$$

which matches that of the theory with untwisted $A_{3}=S U(4)$ gauge group. The coefficient in $S^{4}$ differs from the ordinary expansion by a pure instanton correction. This again shows that the $S L(2, Z)$ fixes correctly the pure instanton correction.

\section{$6.6 E_{6}^{(2)}$ and $D_{4}^{(3)}$}

Let us first consider the $E_{6}^{(2)}$ case. The $\mathcal{G}_{0}$ of the even generators of $E_{6}$ are $F_{4}$. The simple roots of $E_{6}^{(2)}$ are

$$
\boldsymbol{\beta}_{0}=-\boldsymbol{e}_{1}, \quad \boldsymbol{\beta}_{1}=\frac{\boldsymbol{e}_{1}-\boldsymbol{e}_{2}-\boldsymbol{e}_{3}-\boldsymbol{e}_{4}}{2}, \quad \boldsymbol{\beta}_{2}=\boldsymbol{e}_{4}, \quad \boldsymbol{\beta}_{3}=\boldsymbol{e}_{3}-\boldsymbol{e}_{4}, \quad \boldsymbol{\beta}_{4}=\boldsymbol{e}_{2}-\boldsymbol{e}_{3}
$$

Its dual Coxeter number is $h=12$. With $Y_{0}=2 \pi i \tau+\boldsymbol{\beta}_{0}^{*} \cdot \boldsymbol{X}$ and $Y_{a}=\boldsymbol{\beta}_{a}^{*} \cdot \boldsymbol{X}$, we see that the superpotential (5.35) in series is

$$
\begin{aligned}
\mathcal{W}_{E_{6}^{(2)}}= & 2 \sum_{a=0}^{2}\left(y_{a}+2 y_{a}^{2}+3 y_{a}^{3}+4 y_{a}^{4}\right)+\sum_{a=3}^{4}\left(y_{a}+2 y-a^{2}+3 y_{a}^{3}+4 y_{a}^{4}\right) \\
& +2 \sum_{a=0}^{1}\left(y_{a} y_{a+1}+2 y_{a}^{2} y_{a+1}^{2}\right)+\sum_{a=2}^{3}\left(y_{a} y_{a+1}+2 y_{a}^{2} y_{a+1}^{2}\right) \\
& +2 y_{0} y_{1} y_{2}+y_{1} y_{2} y_{3}+y_{2} y_{3} y_{4}++2 y_{2} y_{3}^{2} \\
& +y_{0} y_{1} y_{2} y_{3}+y_{1} y_{2} y_{3} y_{4}+2 y_{1} y_{2} y_{3}^{2}+y_{2} y_{2}^{2} y_{4} \\
& -S\left(-2 \pi i \tau+Y_{0}+2 Y_{1}+3 Y_{2}+4 Y_{3}+2 Y_{4}\right)
\end{aligned}
$$

The glueball superpotential is

$$
\begin{aligned}
\mathcal{W}_{E_{6}^{(2)}}(S ; \tau) & =\mathcal{W}_{E_{6}}^{V Y}+72 S^{2}-756 S^{3}+1490 S^{4} \\
& =\mathcal{W}_{E_{6}}^{V Y}+3 h_{2} S^{2}-14 h_{3} S^{3}+\left(115 h_{4}-\frac{23}{2} \hat{C}_{a b}\left(k_{a}^{*}\right)^{2}\left(k_{b}^{*}\right)^{2}\right) S^{4}+O\left(S^{5}\right)
\end{aligned}
$$

since $h_{2}=24, h_{3}=54, h_{4}=132$, and $\hat{C}_{a b}\left(k_{a}^{*}\right)^{2}\left(k_{b}^{*}\right)^{2}=24$. This matches exactly with the glueball superpotential (6.26) for $E_{6}^{(1)}$ above.

For $D_{4}^{(3)}$ case, the subalgebra $\mathcal{G}_{0}$ of $D_{4}^{(3)}$ is $G_{2}$. The simple roots of $D_{4}^{(3)}$ are

$$
\boldsymbol{\beta}_{0}=-\sqrt{\frac{2}{3}} \boldsymbol{e}_{2}, \quad \boldsymbol{\beta}_{1}=-\sqrt{\frac{1}{2}} \boldsymbol{e}_{1}+\sqrt{\frac{1}{6}} \boldsymbol{e}_{2}, \quad \boldsymbol{\beta}_{2}=\sqrt{2} \boldsymbol{e}_{1}
$$


The dual Coxeter number is $h=6$, which is identical to that of $D_{4}=S O(8)$. With the new variables $Y_{0}=2 \pi i \tau+\boldsymbol{\beta}_{0} \cdot \boldsymbol{X}, Y_{1}=\boldsymbol{\beta}_{1} \cdot \boldsymbol{X}$ and $Y_{2}=\boldsymbol{\beta}_{2} \cdot \boldsymbol{X}$, and $y_{a}=e^{Y_{a}}$, we get the series expansion of the superpotential (5.39) to order $q^{4 / h}$;

$$
\begin{aligned}
\mathcal{W}_{D_{4}^{(3)}=} & 3\left(y_{0}+2 y_{0}^{2}+3 y_{0}^{3}+4 y_{0}^{4}\right)+3\left(y_{1}+2 y_{1}^{2}+3 y_{1}^{3}+4 y_{1}^{4}\right)+y_{2}+2 y_{2}^{2}+3 y_{3}^{3}+4 y_{4}^{4} \\
& +3\left(y_{0} y_{1}+2 y_{0}^{2} y_{1}^{2}\right)+\left(y_{1} y_{2}+2 y_{1}^{2} y_{2}^{2}\right)+y_{1} y_{2}^{2}+3 y_{1} y_{2}^{3}+y_{0} y_{1} y_{2}+y_{0} y_{1} y_{2}^{2} \\
& -S\left(-2 \pi i+Y_{0}+2 Y_{1}+3 Y_{2}\right) .
\end{aligned}
$$

Integrating over the variables $Y_{a}$ leads to the glueball superpotential to order $S^{4}$,

$$
\mathcal{W}_{D_{4}^{(3)}}(S ; \tau)=\mathcal{W}_{D_{4}}^{V Y}+3 h_{2} S^{2}-141 h_{3} S^{3}+115\left(h_{4}-\frac{4}{5}\right) S^{4}+\mathcal{O}\left(S^{5}\right),
$$

where $h_{2}=\sum_{a}\left(k_{a}^{*}\right)^{2}=8, h_{3}=\sum_{a}\left(k_{a}^{*}\right)^{3}=12$, and $h_{4}=\sum_{a}\left(k_{a}^{*}\right)^{4}=20$. This matches exactly with the superpotential (6.26) for $D_{4}^{(1)}$.

\section{Conclusion}

In this work we computed the superpotential of the four dimensional $\mathcal{N}=1^{*}$ theory compactified on $S^{1}$ with twisted boundary condition. The twisting is done with help of outer automorphisms, and because of this, twisted theories arise from twisting of simple-laced groups only. For the case of simple-laced classical Lie algebra, we employed an $\mathrm{M}$ theory construction to argue that all the $\mathcal{N}=4$ supersymmetric counterparts have the $S L(2, Z)$ symmetries. This $S L(2, Z)$ symmetry is expected to be inherited by the corresponding $\mathcal{N}=1^{*}$ theories and acts on the superpotential as modular transformations. We assume that the same holds for $E$ type as well, and used this symmetry to determine the exact superpotential for the twisted case. We explored both symmetry aspects and vacuum physics of these superpotentials, and find no discrepancy.

As the superpotentials do not depend on the compactification radius explicitly, vacuum physics of the gaugino condensate should be unaffected by the twisting. This is partially confirmed by studying the dual glueball superpotentials in the series expansion in the weak coupling regime, whereby we find identical results regardless of twisting or no twisting, adding weights to our proposal of exact superpotential.

As in the case of untwisted theories previously studied, the superpotential for the $\mathcal{N}=1^{*}$ theory with twisted boundary condition is associated with the elliptic Calogero-Moser model. As the physics described by two potentials are identical in the $\mathcal{N}=1^{*}$ theories, such a unity may show up in other context too. Their spectral curves may be identical once the right representation is chosen as the spectral curves 
are supposed to describe the $\mathcal{N}=2^{*}$ physics. There may be a more direct way to obtain the superpotential for the twisted case from the untwisted case by some sort of reduction. The vacua of the $\mathcal{N}=2$ theories compactified on a circle are characterized by hyper-Kähler spaces [15], which may have a similar reduction.

While we have studied here the $\mathcal{N}=1^{*}$ theories with twisted boundary condition, one could imagine general $\mathcal{N}=1$ theories, say with different representations or different interaction, or more general potential, with twisted boundary condition. The full superpotential of the glueball superfield must still be insensitive to the boundary conditions, we anticipate. It would be interesting to check this explicitly. A method to find the superpotential of the compactified theories on a circle has been proposed in Ref. [45], which may be useful along this direction.

Finally, there have been some studies of five dimensional $\mathcal{N}=1$ Yang-Mills theories compactified on a circle [46, 47]. There vacuum structure is closely associated with one-parameter generalization of the Calogero-Moser models 48]. It would be interesting to generalize our consideration to this case also.

\section{Acknowledgements}

H.-U.Y. and S.K. are grateful to Physics Department, University of Texas for hospitality, and S.K. also thanks Chaiho Rim for useful conversations. S.K. is supported in part by BK21 project of the Ministry of Education, Korea, and also by a 2003 Interdisciplinary Research Grant of Seoul National University. K.L. is supported by NSF under Grant No. 0071512, K.L. and H.-U.Y are supported in part by grant No. R01-2003-000-10391-0 from the Basic Research Program of the Korea Science \& Engineering Foundation. P.Y. is supported in part by Korea Research Foundation Grant KRF-2002-070-C00022.

\section{Appendix A: Glueball Superpotential of $B_{r}, C_{r}, G_{2}, F_{4}$ Theories}

While our task, that is, the comparison of glueball superpotentials for twisted

and untwisted cases, is now complete, let us also record those of other untwisted case for the sake of completeness.

Case of $B_{r}$ 
For the $B_{r}=S O(2 r+1)$ gauge theory, the simple roots of the affine algebra are

$$
\boldsymbol{\alpha}_{0}=-\left(\boldsymbol{e}_{1}+\boldsymbol{e}_{2}\right), \boldsymbol{\alpha}_{a}=\boldsymbol{e}_{a}-\boldsymbol{e}_{a+1}, a=1,2, \ldots r-1, \boldsymbol{\alpha}_{r}=\boldsymbol{e}_{r}
$$

with $h=2 r-1$. The superpotential (3.21) in weak coupling expansion is

$$
\begin{aligned}
\mathcal{W}_{B_{r}^{(1)}}= & \sum_{a=0}^{r-1}\left(y_{a}+2 y_{a}^{2}+3 y_{a}^{3}+4 y_{a}^{4}\right)+2\left(y_{r}+2 y_{r}^{2}+3 y_{r}^{3}+4 y_{r}^{4}\right)+y_{0} y_{2}+2 y_{0}^{2} y_{2}^{2} \\
& +\sum_{a=1}^{r-1}\left(y_{a} y_{a+1}+2 y_{a}^{2} y_{a+1}^{2}\right)+y_{0} y_{1} y_{2}+y_{0} y_{2} y_{3}+\sum_{a=1}^{r-2} y_{a} y_{a+1} y_{a+2}+2 y_{r-1}^{2} y_{r} \\
& +y_{0} y_{1} y_{2} y_{3}+y_{0} y_{2} y_{3} y_{4}+\sum_{a=1}^{r-3} y_{a} y_{a+1} y_{a+2} y_{a+3}+y_{r-2} y_{r-1}^{2} y_{r} \\
& -S\left(-2 \pi i \tau+Y_{0}+Y_{1}+2 \sum_{a=1}^{r-1} Y_{a}+Y_{r}\right) .
\end{aligned}
$$

Integrating over $Y_{a}$ 's, the glueball superpotential becomes

$$
\mathcal{W}_{B_{r}^{(1)}}(S ; \tau)=\mathcal{W}_{B_{r}}^{V Y}+(12 r-18) S^{2}-(112 r-224) S^{3}+(1804 r-4232) S^{4}+\mathcal{O}\left(S^{5}\right)
$$

\section{Case of $C_{r}$}

For the $C_{r}=S p(2 r)$ gauge theory, the simple roots of the affine algebra are

$$
\boldsymbol{\alpha}_{0}=-\sqrt{2} \boldsymbol{e}_{1}, \boldsymbol{\alpha}_{a}=\frac{\boldsymbol{e}_{a}-\boldsymbol{e}_{a+1}}{\sqrt{2}}, a=1,2, \ldots r-1, \quad \boldsymbol{\alpha}_{r}=\sqrt{2} \boldsymbol{e}_{r}
$$

The superpotential in weak coupling expansion to order $q^{\frac{4}{h}}$ is

$$
\begin{aligned}
\mathcal{W}_{C_{r}^{(1)}}= & y_{0}+2 y_{0}^{2}+3 y_{0}^{3}+4 y_{0}^{4}+2 \sum_{a=1}^{r-1}\left(y_{a}+2 y_{a}^{2}+3 y_{a}^{3}+4 y_{a}^{4}\right) \\
& +y_{r}+2 y_{r}^{2}+3 y_{r}^{3}+4 y_{4}^{4}+y_{0} y_{1}+2 y_{0}^{2} y_{1}^{2}+2 \sum_{a=1}^{r-2}\left(y_{a} y_{a+1}+2 y_{a}^{2} y_{a+1}^{2}\right) \\
& +y_{r-1} y_{r}+2 y_{r-1}^{2} y_{r}+y_{0} y_{1} y_{2}+2 \sum_{a=1}^{r-3} y_{a} y_{a+1} y_{a+2} \\
& +y_{r-2} y_{r-1} y_{r}+2 y_{0}^{2} y_{1}+2 y_{r-1} y_{r}^{2}+y_{0} y_{1} y_{2} y_{3}+2 \sum_{a=1}^{r-4} y_{a} y_{a+1} y_{a+2} y_{a+3} \\
& +y_{r-3} y_{r-2} y_{r-1} y_{r}+2 y_{0}^{2} y_{1} y_{2}+2 y_{r-2} y_{r-1} y_{r}^{2}-S\left(-2 \pi i \tau+\sum_{a=0}^{r} Y_{a}\right)
\end{aligned}
$$


with the dual Coxeter number $h=r+1$. After integrating over the $Y_{a}$, we obtain the glueball superpotential

$$
\mathcal{W}_{C_{r}^{(1)}}(S ; \tau)=\mathcal{W}^{V Y}+\left(\frac{3 r}{2}+3\right) S^{2}-\left(\frac{7 r}{2}+\frac{35}{4}\right) S^{3}+\left(\frac{115}{8} r+\frac{161}{4}\right) S^{4}+\mathcal{O}\left(S^{5}\right)
$$

\section{Case of $G_{2}$}

The simple roots of $G_{2}^{(1)}$ are

$$
\boldsymbol{\alpha}_{0}=-\sqrt{\frac{1}{2}} \boldsymbol{e}_{1}-\sqrt{\frac{3}{2}} \boldsymbol{e}_{2}, \boldsymbol{\alpha}_{1}=\sqrt{2} \boldsymbol{e}_{1}, \boldsymbol{\alpha}_{2}=-\sqrt{\frac{1}{2}} \boldsymbol{e}_{1}+\sqrt{\frac{1}{6}} \boldsymbol{e}_{2}
$$

The Coxeter number is $h=4$ as $\boldsymbol{\alpha}_{0}+2 \boldsymbol{\alpha}_{1}+\boldsymbol{\alpha}_{2}^{*}=0$. The superpotential in weak coupling expansion is

$$
\begin{aligned}
\mathcal{W}_{G_{2}^{(1)}}= & \sum_{a=0}^{1}\left(y_{a}+2 y_{a}^{2}+3 y_{a}^{3}+4 y_{a}^{4}\right)+3\left(y_{2}+2 y_{2}^{2}+3 y_{2}^{3}+4 y_{4}^{4}\right)+y_{0} y_{1}+2 y_{0}^{2} y_{1}^{2} \\
& +y_{1} y_{2}+2 y_{1}^{2} y_{2}^{2}++y_{0} y_{1} y_{2}+y_{1}^{2} y_{2}+3 y_{1}^{3} y_{2}-6 y_{0} y_{1}^{2} y_{2} \\
& -S\left(-2 \pi i \tau+Y_{0}+2 Y_{1}+Y_{2}\right) .
\end{aligned}
$$

Here the term $-6 y_{0} y_{1}^{2} y_{2}$ is due to the contribution of a pure single instanton. The glueball superpotential is

$$
\mathcal{W}_{G_{2}^{(1)}}(S ; \tau)=\mathcal{W}_{G_{2}}^{V Y}+\frac{40}{3} S^{2}-\frac{700}{9} S^{3}+\frac{66632}{81} S^{4}+O\left(S^{5}\right)
$$

\section{Case of $F_{4}$}

The simple roots of $F_{4}^{(1)}$ are

$$
\boldsymbol{\alpha}_{0}=-e_{1}-e_{2}, \boldsymbol{\alpha}_{1}=e_{2}-e_{3}, \boldsymbol{\alpha}_{2}=e_{3}-e_{4}, \boldsymbol{\alpha}_{3}=e_{4}, \boldsymbol{\alpha}_{4}=\frac{e_{1}-e_{2}-e_{3}-e_{4}}{2}
$$

The dual Coxeter number is $h=9$. With $Y_{0}=2 \pi i \tau+\boldsymbol{\alpha}_{0} \cdot \boldsymbol{X}$ and $Y_{a}=\boldsymbol{\alpha}_{a}^{*} \cdot \boldsymbol{X}$, the superpotential becomes

$$
\begin{aligned}
\mathcal{W}_{F_{4}^{(1)}}= & \sum_{a=0}^{2}\left(y_{a}+2 y_{a}^{2}+3 y_{a}^{3}+4 y_{a}^{4}\right)+2 \sum_{a=3}^{4}\left(y_{a}+2 y_{a}^{2}+3 y_{a}^{3}+4 y_{a}^{4}\right)+\sum_{a=0}^{2}\left(y_{a} y_{a+1}+2 y_{a}^{2} y_{a+1}^{2}\right) \\
& +2\left(y_{3} y_{4}+2 y_{3}^{2} y_{4}^{2}\right)+y_{0} y_{1} y_{2}+y_{1} y_{2} y_{3}+2 y_{2}^{2} y_{3}+y_{2} y_{3} y_{4}+y_{0} y_{1} y_{2} y_{3} \\
& +y_{1} y_{2}^{2} y_{3}+y_{1} y_{2} y_{3} y_{4}+2 y_{2}^{3} y_{3} y_{4}-S\left(-2 \pi i \tau+Y_{0}+2 Y_{1}+3 Y_{2}+2 Y_{3}+Y_{4}\right) . \quad \text { (A.11) }
\end{aligned}
$$

The glueball superpotential for $F_{4}^{(1)}$ becomes

$$
\mathcal{W}_{F_{4}^{(1)}}(S ; \tau)=\mathcal{W}_{F_{4}}^{V Y}+45 S^{2}-\frac{1575}{4} S^{3}+\frac{25875}{4} S^{4}+\mathcal{O}\left(S^{5}\right) \text {. }
$$




\section{Appendix B: Lie Algebra}

We choose an orthonormal basis $\left\{T^{i}\right\}$ of a simple Lie algebra $\mathcal{G}$ of rank $r$ with respect to the group invariant inner product defined in the adjoint representation,

$$
\operatorname{Tr}\left(T^{i}, T^{j}\right)=\frac{1}{C_{2}(\mathcal{G})} \operatorname{tr} T^{i} T^{j}=\delta^{i j},
$$

with a normalization coefficient $C_{2}(\mathcal{G})$. (Here tr is a matrix trace and $\operatorname{Tr}$ is a just notation for the inner product defined as above.) On this basis, the second Casimir operator is $C_{2}(\mathcal{G})=\sum_{i}\left(T^{i}\right)^{2}$ in the adjoint representation. A maximal set $\boldsymbol{H}=$ $\left(H_{1}, H_{2}, \ldots H_{r}\right)$ of mutually commuting generators $H_{a}$, where $a=1,2, \ldots r$, forms an $r$-dimensional vector. The rest of generators can be combined as step operators $E_{\boldsymbol{\alpha}}$ such that

$$
\left[\boldsymbol{H}, E_{\boldsymbol{\alpha}}\right]=\boldsymbol{\alpha} E_{\alpha}
$$

with the root vector $\boldsymbol{\alpha}$ in $r$-dimension. With its hermitian dual $E_{-\alpha}=E_{\alpha}^{\dagger}$, it satisfies

$$
\left[E_{\boldsymbol{\alpha}}, E_{\boldsymbol{\alpha}}^{\dagger}\right]=\boldsymbol{\alpha}^{*} \cdot \boldsymbol{H}
$$

where $\boldsymbol{\alpha}^{*}$ is the co-root defined by

$$
\boldsymbol{\alpha}^{*}=\frac{2 \boldsymbol{\alpha}}{\boldsymbol{\alpha}^{2}}
$$

We choose the normalization so that the longest roots have the length square two. It is known that the normalization coefficient becomes

$$
C_{2}(\mathcal{G})=2 h
$$

with the dual Coxeter number $h$ defined later.

There is a set of simple roots $\boldsymbol{\alpha}_{a}, a=1,2, \ldots r$ which spans the root lattice $\Lambda_{R}$. The lowest negative root is denoted by $\boldsymbol{\alpha}_{0}$, which turns out to be a long root. With our normalization $\boldsymbol{\alpha}_{0}^{2}=2$. The co-roots of the simple roots span the co-root lattice $\Lambda_{R}^{*}$. The weight lattice $\Lambda_{W}$ is spanned by fundamental weights $\boldsymbol{w}_{a}$ satisfying $\boldsymbol{w}_{a} \cdot \boldsymbol{\alpha}_{b}^{*}=\delta_{a b}$. The co-weight lattice $\Lambda_{W}^{*}$ is spanned by the fundamental co-weights $\boldsymbol{w}_{a}^{*}=\left(2 / \boldsymbol{\alpha}_{a}^{2}\right) \boldsymbol{w}_{a}$. For any simple Lie algebra, the length of roots have at most two different values. Our normalization implies that any root in a simply laced algebra has length square two, and any long root in a non-simply laced algebra has length square two. The extended Dynkin diagram of Lie algebra $\mathcal{G}$ is defined by $\boldsymbol{\alpha}_{a}, a=0,1, \ldots r$ and identical to the Dynkin diagram of the corresponding untwisted affine algebra $\mathcal{G}^{(1)}$ which is discussed in the next appendix. They are shown in Figure 3 , where the solid red dots denote $\boldsymbol{\beta}_{0}$. 
There exists a unique set of positive integers $k_{a}^{*}$, the so-called co-marks, such that $k_{0}^{*}=1$ and

$$
\sum_{a=0}^{r} k_{a}^{*} \boldsymbol{\alpha}_{a}^{*}=0
$$

It is minimal in the sense that there is no smaller positive integers such that the above equation is true. The dual Coxeter number is defined as

$$
h=\sum_{a=0}^{r} k_{a}^{*}
$$

which is always bigger than the rank $r$ of the algebra. These comarks for the corresponding roots are shown in the Figure 3.

For a given root $\boldsymbol{\alpha}$, a $S U(2)$ subalgebra is defined by three generators,

$$
t^{1}=\frac{1}{2}\left(E_{\alpha}+E_{\alpha}^{\dagger}\right), t^{2}=\frac{1}{2 i}\left(E_{\alpha}-E_{\alpha}^{\dagger}\right), t^{3}=\frac{1}{2} \boldsymbol{\alpha}^{*} \cdot \boldsymbol{H},
$$

which satisfy the $S U(2)$ algebra $\left[t^{i}, t^{j}\right]=i \epsilon^{i j k} t^{k}$.

The Weyl vector $\rho=\sum_{i=1}^{r} \boldsymbol{w}_{i}$ and the level vector $\rho^{*}=\sum_{i=1}^{r} \boldsymbol{w}_{i}^{*}$ of a Lie algebra $\mathcal{G}$ are related to the set of all positive roots, $\mathcal{R}_{+}(\mathcal{G})$, by the following equations,

$$
\begin{aligned}
& \boldsymbol{\rho}=\sum_{i=1}^{r} \boldsymbol{w}_{i}=\frac{1}{2} \sum_{\boldsymbol{\alpha} \in \mathcal{R}_{+}(\mathcal{G})} \boldsymbol{\alpha}, \\
& \boldsymbol{\rho}^{*}=\sum_{i=1}^{r} \boldsymbol{w}_{i}^{*}=\frac{1}{2} \sum_{\boldsymbol{\alpha} \in \mathcal{R}_{+}(\mathcal{G})} \boldsymbol{\alpha}^{*} .
\end{aligned}
$$

The level $l(\lambda)$ and co-level $l^{*}(\lambda)$ of a weight $\lambda$ are defined by

$$
l(\lambda)=\lambda \cdot \boldsymbol{\rho}^{*}, \quad l^{*}(\lambda)=\lambda \cdot \boldsymbol{\rho},
$$

respectively. All roots take the integer levels and all co-roots the integer co-levels. Note that the dual Coxeter number is related the co-level of $\boldsymbol{\alpha}_{0}^{*}$,

$$
h=1-\boldsymbol{\alpha}_{0}^{*} \cdot \rho .
$$

While $\boldsymbol{\alpha}_{0}$ has the lowest level, its coroot $\boldsymbol{\alpha}_{0}^{*}$ does not have the lowest co-level, or the lowest root of the co-root system. However the values

$$
\boldsymbol{\alpha}^{*} \cdot \rho-\frac{2 h}{\boldsymbol{\alpha}^{2}},
$$

for all roots $\boldsymbol{\alpha}$ takes the maximum value one for the positive root $-\boldsymbol{\alpha}_{0}$. 


\section{Appendix C: Untwisted Affine Lie Algebra}

We start with a simple Lie algebra $\mathcal{G}$ of rank $r$ with generators $T^{i}$. By attaching integer-valued gradings $n$ to these generators, we find the corresponding untwisted affine Lie algebra $\mathcal{G}^{(1)}$ of infinite number of generators $T_{n}^{i}$, which satisfies

$$
\left[T_{m}^{i}, T_{n}^{j}\right]=i f^{i j k} T_{m+n}^{k}+p n \delta^{i j} \delta_{m+n, 0}
$$

The constant $p$ is a central charge which commutes with all elements of $\mathcal{G}^{(1)}$, and it will vanish in all cases of our present interest. From the Cartan subalgebra of $\mathcal{G}$, we choose the corresponding commuting operators $\left(H_{0}^{1}, H_{0}^{2}, \ldots H_{0}^{r}\right)$ in the zero-grading. In addition we introduce the operator $d$ which measures the grading,

$$
\left[d, T_{n}^{i}\right]=n T_{n}^{i}
$$

Then roots of the untwisted affine Lie algebra $\mathcal{G}^{(1)}$ are $(r+1)$-component vectors composed of the eigenvalues of $\left\{H_{0}^{a}, d\right\}$. It is not difficult to check that a simple root system of $\hat{\mathcal{G}}$, of which every root is either all positive or all negative linear combination, is provided by

$$
\left(\boldsymbol{\alpha}_{a}, 0\right), a=1, \ldots r, \quad\left(\boldsymbol{\alpha}_{0}, 1\right)
$$

where $\boldsymbol{\alpha}_{a}\left(\boldsymbol{\alpha}_{0}\right)$ are simple roots (the lowest negative root) of $\mathcal{G}$. The Dynkin diagram shown in Figure 3 of the untwisted affine algebra $\mathcal{G}^{(1)}$ is made of $\left\{\boldsymbol{\alpha}_{a}\right\}, i=0,1, \ldots, r$ and $\boldsymbol{\alpha}_{0}$, and so identical to the extended Dynkin diagram of the original Lie algebra $\mathcal{G}$. For each positive root $\boldsymbol{\alpha}$ and grading $n \in Z$, there exist a raising operator $E_{\alpha}^{n}$ and a lowering operator $\left(E_{\alpha}^{n}\right)^{\dagger}=E_{-\alpha}^{-n}$. With $\boldsymbol{\alpha}^{*} \cdot \boldsymbol{H}_{0}$, these operators define three generators for the $S U(2)$ algebra,

$$
t^{1}=\frac{1}{2}\left(E_{\alpha}^{n}+\left(E_{\alpha}^{n}\right)^{\dagger}\right), \quad t^{2}=\frac{1}{2 i}\left(E_{\alpha}^{n}-\left(E_{\alpha}^{n}\right)^{\dagger}\right), t^{3}=\frac{1}{2} \boldsymbol{\alpha}^{*} \cdot \boldsymbol{H}_{0} .
$$

\section{Appendix D: Twisted Affine Lie Algebra}

A twisted affine Lie algebra, $\mathcal{G}^{(L)}$, out of an ordinary simple Lie algebra $\mathcal{G}$ of rank $r$, is based on an outer automorphism $\boldsymbol{\sigma}$ of $\mathcal{G}$ such that $\boldsymbol{\sigma}^{L}=1$ with the smallest positive integer $L$. First we divide elements of the complex vector space $\mathcal{G}$ into

subspaces $\mathcal{G}_{n}, n=0, \ldots L-1$, by their eigenvalues $e^{\frac{2 \pi i n}{L}}$ with respect to $\tau$. Because $\boldsymbol{\sigma}$ is an automorphism, it preserves the group and algebraic relations. Thus,

$$
\boldsymbol{\sigma}\left(\left[\mathcal{G}_{n}, \mathcal{G}_{n^{\prime}}\right]\right)=\left[\boldsymbol{\sigma}\left(\mathcal{G}_{n}\right), \boldsymbol{\sigma}\left(\mathcal{G}_{n^{\prime}}\right)\right]=e^{\frac{2 \pi i\left(n+n^{\prime}\right)}{L}}\left[\mathcal{G}_{n}, \mathcal{G}_{n^{\prime}}\right]
$$


which means that $\left[\mathcal{G}_{n}, \mathcal{G}_{n^{\prime}}\right] \subset \mathcal{G}_{n+n^{\prime} \bmod L}$. Note that the subspace $\mathcal{G}_{0}$ is special as it is a Lie algebra by itself of rank $r^{\prime}<r$. All other subspaces $\mathcal{G}_{n}, n \neq 0$ form representations of the Lie algebra $\mathcal{G}_{0}$.

The original elements of the Lie algebra can be expressed as linear combinations of the elements $T_{\frac{n}{L}}^{i}$ of these subspaces $\mathcal{G}_{n}$, whose structure constant would be $f_{n, n^{\prime}}^{i j k}$ We now assign integer gradings to each of these elements of $\mathcal{G}_{n}$ to obtain generators $T_{s}^{i}$ with $s=\frac{n}{L}+m$, and require them to satisfy the commutation relation of the twisted affine Lie algebra $\mathcal{G}^{(L)}$,

$$
\left[T_{s}^{i}, T_{s^{\prime}}^{j}\right]=i f_{n, n^{\prime}}^{i j k} T_{s+s^{\prime}}^{k}+p s \delta^{a b} \delta_{s+s^{\prime}, 0}
$$

which is consistent with the original Lie algebra. The central term, $p$, vanishes for the case we study here.

The outer automorphism $\boldsymbol{\sigma}$ modulo inner automorphism is known to be equivalent to the exchanging operator of simple roots $\boldsymbol{\alpha}_{a}, a=1,2, \ldots r$ of the Lie algebra $\mathcal{G}$ according to the symmetry of its Dynkin (not extend Dynkin) diagram. Thus the exchange transformation $\boldsymbol{\sigma}$ defines the outer automorphism of the raising operators of simple roots as

$$
\boldsymbol{\sigma}\left(E_{\boldsymbol{\alpha}_{a}}\right)=E_{\boldsymbol{\sigma}\left(\boldsymbol{\alpha}_{a}\right)},
$$

It is straightforward to extend this outer automorphism to the raising operators of other positive roots as they are given by the commutations of $E_{\boldsymbol{\alpha}_{a}}$. Also one obtains the $\boldsymbol{\sigma}$ transformation of the lowering operators by noting $\boldsymbol{\sigma}\left(E_{\boldsymbol{\alpha}_{a}}^{\dagger}\right)=E_{\boldsymbol{\sigma}\left(\boldsymbol{\alpha}_{a}\right)}^{\dagger}$. The extension to the Cartan subalgebra can be obtained by the commutation relation between raising and lowering operators. As we know the transformation of all generators of $\mathcal{G}$, we can construct the eigen operator space $\mathcal{G}_{n}$ of the outer automorphism by linear combination of operators.

The exchange symmetry of simple roots in the Dynkin diagram can be easily extended to the exchange symmetries of all roots. It preserves the length of the root and the angle between the basis $\boldsymbol{\alpha}_{a}$. Thus it can be regarded as an element of the orthogonal group $O(r)$. We use the same notation $\boldsymbol{\sigma}$ for both the outer automorphism and this linear map without confusion. From the outer automorphism of the commutation (B.3), we get

$$
\left(\left[E_{\boldsymbol{\sigma}\left(\boldsymbol{\alpha}_{a}\right)}, E_{\boldsymbol{\sigma}\left(\boldsymbol{\alpha}_{a}\right)}^{\dagger}\right]=\boldsymbol{\sigma}\left(\boldsymbol{\alpha}_{a}^{*} \cdot \boldsymbol{H}\right)=\boldsymbol{\sigma}\left(\boldsymbol{\alpha}_{a}^{*}\right) \cdot \boldsymbol{H},\right.
$$

which implies $\boldsymbol{\sigma}(\boldsymbol{\alpha} \cdot \boldsymbol{H})=\boldsymbol{\sigma}(\boldsymbol{\alpha}) \cdot \boldsymbol{H}$. This is consistent with the outer automorphism of a variation $\left[\boldsymbol{\beta} \cdot \boldsymbol{H}, E_{\alpha_{i}}\right]=\boldsymbol{\beta} \cdot \boldsymbol{\alpha} E_{\alpha_{i}}$ of the commutation relation (B.2), which is

$$
\left[\boldsymbol{\sigma}(\boldsymbol{\beta} \cdot \boldsymbol{H}), E_{\boldsymbol{\sigma}\left(\boldsymbol{\alpha}_{a}\right)}\right]=\boldsymbol{\beta} \cdot \boldsymbol{\alpha}_{a} E_{\boldsymbol{\sigma}\left(\alpha_{a}\right)} .
$$


The left hand side of the above equation is

$$
\left[\boldsymbol{\sigma}(\boldsymbol{\beta}) \cdot \boldsymbol{H}, E_{\boldsymbol{\sigma}\left(\alpha_{i}\right)}\right]=\boldsymbol{\sigma}(\boldsymbol{\beta}) \cdot \boldsymbol{\sigma}\left(\boldsymbol{\alpha}_{i}\right) E_{\boldsymbol{\sigma}\left(\alpha_{i}\right)},
$$

which is identical to the previous equation as $\boldsymbol{\sigma}(\boldsymbol{\beta} \cdot \boldsymbol{\alpha})=\boldsymbol{\beta} \cdot \boldsymbol{\alpha}$.

Thus the elements of the Cartan subalgebra which belong to $\mathcal{G}_{0}$ would be made of $\boldsymbol{\sigma}(\boldsymbol{\zeta} \cdot \boldsymbol{H})=\boldsymbol{\zeta} \cdot \boldsymbol{H}$ or $\boldsymbol{\sigma}(\boldsymbol{\zeta})=\boldsymbol{\zeta}$. So $\mathcal{G}_{0}$ will be a $r^{\prime}$ dimensional Lie algebra whose Cartan algebra is spanned by $\sum_{l=0}^{L} \boldsymbol{\sigma}^{l}\left(\boldsymbol{\alpha}_{a}\right) \cdot \boldsymbol{H}, a=1,2, \ldots r$. Similarly, $\sum_{l=0}^{L} e^{-2 \pi i l n / L} \boldsymbol{\sigma}^{l}\left(\boldsymbol{\alpha}_{a}\right)$. $\boldsymbol{H}, i=1,2 \ldots r$ would belong to $\mathcal{G}_{n}$. Since $\left[\boldsymbol{H}, E_{\boldsymbol{\sigma}(\alpha)}\right]=\boldsymbol{\sigma}(\boldsymbol{\alpha}) \boldsymbol{\sigma}\left(E_{\alpha}\right)$, we get $\boldsymbol{\sigma}\left(E_{\alpha}\right)=$ $\epsilon(\boldsymbol{\sigma}, \alpha) E_{\boldsymbol{\sigma}(\alpha)}$ such that $\epsilon\left(\boldsymbol{\sigma}^{L}, \alpha\right)=1$ as $\boldsymbol{\sigma}^{L}=1$. Thus the linear combinations $\sum_{l=1}^{L} e^{-2 \pi i n l / L} \boldsymbol{\sigma}^{l}\left(E_{\alpha}\right)$ would belong to $\mathcal{G}_{n}$.

For any root vector $\boldsymbol{\alpha}, \sum_{l=1}^{L} \boldsymbol{\sigma}^{l}\left(\boldsymbol{\alpha}_{a}\right) \cdot \boldsymbol{\alpha}=\sum_{l=1}^{L} \boldsymbol{\sigma}^{l}\left(\boldsymbol{\alpha}_{a}\right) \cdot \frac{1}{L} \sum_{l=1}^{L} \boldsymbol{\sigma}^{l}(\boldsymbol{\alpha}) / L$. Thus we see that

$$
\left[\sum_{l=1}^{L} \boldsymbol{\sigma}^{l}\left(\boldsymbol{\alpha}_{a}\right) \cdot \boldsymbol{H}, \sum_{l=1}^{L} e^{-2 \pi i n l / L} \boldsymbol{\sigma}^{l}\left(E_{\boldsymbol{\alpha}}\right)\right]=\left(\sum_{l=1}^{L} \boldsymbol{\sigma}^{l}\left(\boldsymbol{\alpha}_{a}\right) \cdot \frac{1}{L} \sum_{l=1}^{L} \boldsymbol{\sigma}^{l}(\boldsymbol{\alpha})\right) \sum_{l=1}^{L} e^{-2 \pi i n l / L} \boldsymbol{\sigma}^{l}\left(E_{\alpha}\right) .
$$

This means that the weight of root vectors of an element $\sum_{l=1}^{L} e^{-2 \pi i n l / L} E_{\boldsymbol{\sigma}^{l}(\alpha)}$ in $\mathcal{G}_{n}$ is given in $\frac{1}{L} \sum_{l=1}^{L} \boldsymbol{\sigma}^{l}(\boldsymbol{\alpha})$.

Now we have the grading of the Lie algebra due to the outer automorphism explicitly and so we can define the twisted affine algebra $\mathcal{G}^{(L)}$. The maximally commuting subalgebra of $\mathcal{G}^{(L)}$ would be a $r^{\prime}$ vector $\boldsymbol{H}^{\prime}=\left\{\frac{1}{L} \sum_{l} \boldsymbol{\sigma}^{l}\left(\boldsymbol{\alpha}_{a}\right) \cdot \boldsymbol{H}_{0}\right\}$ of $\mathcal{G}_{0}$ and the grading operator $d$ whose eigenvalue is $s=m+n / L$. The simple roots of $\mathcal{G}^{(L)}$ would be

$$
\left\{\left(\boldsymbol{\beta}_{a}, 0\right),\left(\boldsymbol{\beta}_{0}, \frac{1}{L}\right)\right\}=\left\{\left(\frac{1}{L} \sum_{l=1}^{L} \boldsymbol{\sigma}^{l}\left(\boldsymbol{\alpha}_{i}\right), 0\right),\left(\boldsymbol{\beta}_{0}, \frac{1}{L}\right)\right\},
$$

where $j=1,2, \ldots r^{\prime}$ and $\boldsymbol{\beta}_{0}$ is the lowest negative weight of $\mathcal{G}_{1}$ with respect to $\mathcal{G}_{0}$. The simple roots of sub Lie algebra $\mathcal{G}_{0}$ are $\boldsymbol{\beta}_{a}, a=1,2, \ldots r^{\prime}$. These simple roots $\boldsymbol{\beta}_{a}, a=0,1,2, \ldots r^{\prime}$ of the twisted affine algebra $\mathcal{G}^{(L)}$ define the Dynkin diagram of the twisted affine Lie algebra, which is shown in Figure 3.

\section{Appendix E: Dynkin Diagrams and Some New Identities}

The nontrivial outer automorphism is possible only for simply laced group with symmetric Dynkin diagram. For $A_{r}=S U(r+1), D_{r+1}=S O\left(2(r+1)\right.$ and $E_{6}$, the order of the symmetry is $L=2$. For $D_{4}=S O(8)$, there is also the triple symmetry of order $L=3$. For all simple Lie algebra $\mathcal{G}$, their untwisted affine algebra is denoted as $\mathcal{G}^{(1)}$, and their twisted affine algebra would be denoted as $\mathcal{G}^{(L)}$. Their Dynkin 
diagram is given in Figure 3. Note that the lowest root $\boldsymbol{\alpha}_{0}$ in all untwisted affine algebra which appears as a filled circle is a long root. The root $\boldsymbol{\beta}_{0}$ in $A_{2 r}^{(2)}$ case is a long root. For all other twisted affine case the root $\boldsymbol{\beta}_{0}$ is a short root.

Once we fix the normalization of the elements of the Lie algebra $\mathcal{G}$ as in Appendix $\mathrm{B}$, which fixes the length of the root, the procedure in the previous appendix fixes the normalization of the elements of untwisted and twisted affine algebra. For our normalization the long roots of the untwisted affine algebra would have length square two. This leads to the fact that for all twisted affine algebras, the longest root has square length two.

For a Lie group $\mathcal{G}$ of rank $r$, its extended Dynkin diagram of simple roots $\boldsymbol{\alpha}_{a}, a=$ $1,2 \ldots r$ and the lowest root $\boldsymbol{\alpha}_{0}$ is that of the corresponding untwisted affine algebra. Similarly the simple roots $\boldsymbol{\beta}_{a}, a=0,1, \ldots r^{\prime}$ defines the Dynkin diagram of the twisted affine algebra $\mathcal{G}^{(L)}$. One can see that the dual simple roots $\boldsymbol{\alpha}_{a}^{*}, a=0,1, \ldots r$ of untwisted affine algebra defines the dual Dynkin diagram of untwisted affine algebra. Similarly the dual of simple roots $\boldsymbol{\beta}_{a}^{*}, a=0,1, \ldots r^{\prime}$ defines the dual Dynkin diagram of the twisted affine algebra. The Dynkin diagrams of $B_{r}^{(1)}$ and $A_{2 r-1}^{(2)}$ are dual to each other. The same is true for pairs, $\left(C_{r}^{(1)}, D_{r+1}^{(2)}\right),\left(F_{4}^{(1)}, E_{6}^{(2)}\right)$, and $\left(G_{2}^{(1)}, D_{4}^{(2)}\right)$. The rest of Dynkin diagrams in Figure 3 are self-dual.

For untwisted Lie algebra $\mathcal{G}^{(1)}$ for a Lie group $\mathcal{G}$ of rank $r$, their simple roots are $\boldsymbol{\alpha}_{a}, i=0,1, \ldots r$, which appear in the extended Dynkin diagram of $\mathcal{G}$. As defined in Appendix A, the comarks $k_{a}^{*}$ and the dual Coxeter number $h\left(\mathcal{G}^{(1)}\right)$ are defined so that $k_{0}^{*}=1$, and

$$
\sum_{a=0}^{r} k_{a}^{*} \boldsymbol{\alpha}_{a}^{*}=0, \quad h\left(\mathcal{G}^{(1)}\right)=\sum_{a=0}^{r} k_{a}^{*} .
$$

For twisted Lie algebra $\mathcal{G}^{(L)}$ for a Lie group $\mathcal{G}$ of rank $r$, their simple roots are $\boldsymbol{\beta}_{a}, a=0,1, \ldots, r^{\prime}$ with $r^{\prime}<r$. One can define the co-marks $\tilde{k}_{a}^{*}$ and the dual Coxeter number $h\left(\mathcal{G}^{(L)}\right)$ such that

$$
\sum_{a=0}^{r^{\prime}} \tilde{k}_{a}^{*} \boldsymbol{\beta}_{a}^{*}=0, \quad h\left(\mathcal{G}^{(L)}\right)=\sum_{a=0}^{r^{\prime}} \tilde{k}_{a}^{*},
$$

where $\tilde{k}_{0}^{*}=2$ is for $A_{2 r}^{(2)}$ and $\tilde{k}_{0}^{*}=1$ for the rest of twisted affine algebra.

There are four identities for the root systems of untwisted and twisted affine algebra we notice here. It is straight-forward to prove them by going over the case by the case.

- Fact 1 : The dual Coxeter number does not change with twisting. For a Lie algebra $\mathcal{G}$ which has nontrivial outer automorphism, the dual Coxeter number 




Figure 3: Dynkin Diagrams for Affine Algebra with comarks

for the untwisted case is identical to that of the twisted case,

$$
h\left(\mathcal{G}^{(1)}\right)=h\left(\mathcal{G}^{(L)}\right)
$$

Thus we denote the dual Coxeter number by $h$ regardless of the twisting.

- Fact 2 : The above construction of simple roots $\boldsymbol{\beta}_{a}$ 's from $\boldsymbol{\alpha}_{a}$ 's comes with a particular relative normalization between generators of $\mathcal{G}^{(1)}$ and those of 
$\mathcal{G}^{(L)}$. This choice is especially convenient because it seems to imply a universal relationship of the form,

$$
\frac{2}{\boldsymbol{\alpha}_{0}^{2}}=\frac{1}{1} \frac{2 k_{0}^{*}}{\boldsymbol{\alpha}_{0}^{2}}=\frac{1}{L} \frac{2 \tilde{k}_{0}^{*}}{\boldsymbol{\beta}_{0}^{2}}
$$

regardless of which Lie algebra we started with. Note that $k_{0}^{*}=1$.

- Fact 3 : With the same normalization convention, another nontrivial and universal identity holds,

$$
\prod_{a=0}^{r}\left[\frac{k_{a}^{*} \boldsymbol{\alpha}_{a}^{2}}{2}\right]^{k_{a}^{*}}=\prod_{a=0}^{r^{\prime}}\left[\frac{\tilde{k}_{a}^{*} \boldsymbol{\beta}_{a}^{2}}{2}\right]^{\tilde{k}_{a}^{*}}
$$

This quantity is one for the Lie algebra $A_{r}=S U(r+1)$.

- Fact 4 : For a Lie algebra $\mathcal{G}$ with outer automorphism, the number of positive roots of the Lie algebra $\mathcal{G}$ of $\mathcal{G}^{(1)}$ is related to the number of postive roots of $\mathcal{G}_{0}$ of the twisted affine algebra $\mathcal{G}^{(2)}$ as follows. Here we use the convention the longest roots of the (twisted) affine algebra have length square two.

$$
\text { \# of positive roots of } \mathcal{G}=\sum_{\text {all positive } \operatorname{roots} \boldsymbol{\beta} \text { of } \mathcal{G}_{0}} \frac{2}{\boldsymbol{\beta}^{2}} \text { for all } \mathcal{G}^{(2)}
$$

which holds in all cases with the exception of $A_{2 r}^{(2)}$. For the latter we have instead

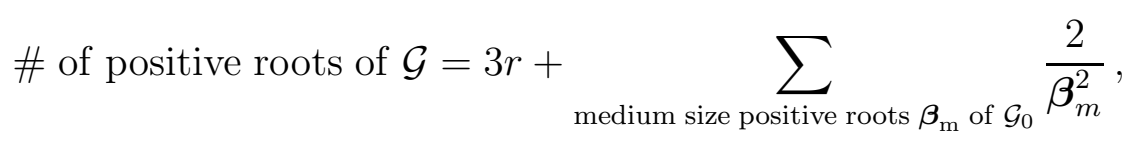

where $\boldsymbol{\beta}_{m}$ 's are the medium size roots introduced in subsection (5.2).

\section{Appendix F: Elliptic Functions}

The Weierstrass elliptic function is

$$
\wp(z ; 2 \pi i, 2 \pi i \tau) \equiv \frac{1}{z^{2}}+\sum_{m_{1}, m_{2} \in \mathbb{Z}}^{\prime}\left\{\frac{1}{\left(z+2 \pi i m_{1}+2 \pi i \tau m_{2}\right)^{2}}-\frac{1}{\left(2 \pi i m_{1}+2 \pi i \tau m_{2}\right)^{2}}\right\}
$$

where the prime in the sum means to exclude the point $\left(m_{1}, m_{2}\right)=(0,0)$. This function is even under $z \rightarrow-z$ and doubly periodic under $z \rightarrow z+2 \pi i m+2 \pi i \tau n$ with $m, n \in \mathbb{Z}$. It has a double pole at $z=0$. We can choose $\operatorname{Im}(\tau)>0$. We 
use a simpler notation $\wp(z)=\wp(z ; 2 \pi i, 2 \pi i \tau)$ unless we have different periods. The Weierstrass elliptic function can be represented by a sum

$$
\wp(z)=\frac{1}{12}+\sum_{n=-\infty}^{\infty} \frac{1}{q^{n} y+q^{-n} y^{-1}-2}-\sum_{n=1}^{\infty} \frac{2}{q^{n}+q^{-n}-2},
$$

where

$$
q=e^{2 \pi i \tau}, \quad y=e^{z}
$$

For small $q$ and $e^{z}$, we can expand the above expression in a series

$$
\wp(z)=\frac{1}{12}+\sum_{k=1}^{\infty} k y^{k}+\sum_{k=1}^{\infty} \sum_{n=1}^{\infty} k q^{k n}\left(y^{k}+y^{-k}-2\right) .
$$

The Weierstrass elliptic function has the $S L(2, \mathbb{Z})$ symmetry under which

$$
\wp(z ; 2 \pi i(c \tau+d), 2 \pi i(a \tau+b))=\wp(z ; 2 \pi i, 2 \pi i \tau)
$$

for all $a, b, c, d \in \mathbb{Z}$ and $a d-b c=1$. Thus the $S L(2, Z)$ transformation

$$
\begin{gathered}
\tau \rightarrow \frac{a \tau+b}{c \tau+d}, \\
z \rightarrow \frac{z}{c \tau+d},
\end{gathered}
$$

leads to a modular function of weight two,

$$
\wp(z ; 2 \pi i, 2 \pi i \tau) \rightarrow(c \tau+d)^{2} \wp(z ; 2 \pi i, 2 \pi i \tau) .
$$

The Weierstrass elliptic function satisfies an obvious homogeneity relation,

$$
\wp(x z ; 2 \pi i x, 2 \pi i \tau x)=\frac{1}{x^{2}} \wp(z ; 2 \pi i, 2 \pi i \tau),
$$

which allows

$$
\wp(z ; \pi i, 2 \pi i \tau)=4 \wp(2 z ; 2 \pi i, 4 \pi i \tau)
$$

In addition it satisfies a double-period formula

$$
\wp(z ; \pi i, 2 \pi i \tau)=\wp(z)+\wp(z+\pi i)-\wp(\pi i)
$$

and a triple period formula

$$
\wp(z, 2 \pi i / 3,2 \pi i \tau)=\wp(z)+\wp\left(z+\frac{2 \pi i}{3}\right)+\wp\left(z+\frac{4 \pi i}{3}\right)-\wp\left(\frac{2 \pi i}{3}\right)-\wp\left(\frac{4 \pi i}{3}\right) .
$$




\section{References}

[1] C. Vafa and E. Witten, "A Strong coupling test of S duality," Nucl. Phys. B 431, 3 (1994) [arXiv:hep-th/9408074].

[2] N. Seiberg and E. Witten, "Electric - magnetic duality, monopole condensation, and confinement in $\mathrm{N}=2$ supersymmetric Yang-Mills theory," Nucl. Phys. B 426, 19 (1994) [Erratum-ibid. B 430, 485 (1994)] [arXiv:hep-th/9407087].

N. Seiberg and E. Witten, "Monopoles, duality and chiral symmetry breaking in N=2 supersymmetric QCD," Nucl. Phys. B 431, 484 (1994) [arXiv:hep-th/9408099].

[3] R. Donagi and E. Witten, "Supersymmetric Yang-Mills Theory And Integrable Systems," Nucl. Phys. B 460, 299 (1996) [arXiv:hep-th/9510101].

[4] A. Gorsky, I. Krichever, A. Marshakov, A. Mironov and A. Morozov, "Integrability and Seiberg-Witten exact solution," Phys. Lett. B 355, 466 (1995) [arXiv:hep-th/9505035].

[5] E. J. Martinec and N. P. Warner, "Integrable systems and supersymmetric gauge theory," Nucl. Phys. B 459, 97 (1996) [arXiv:hep-th/9509161].

[6] E. J. Martinec, "Integrable Structures in Supersymmetric Gauge and String Theory," Phys. Lett. B 367, 91 (1996) [arXiv:hep-th/9510204].

[7] A. Marshakov, "On integrable systems and supersymmetric gauge theories," Theor. Math. Phys. 112, 791 (1997) [Teor. Mat. Fiz. 112N1, 3 (1997)] [arXiv:hep-th/9702083].

[8] T. Nakatsu and K. Takasaki, "Whitham-Toda hierarchy and N = 2 supersymmetric Yang-Mills theory," Mod. Phys. Lett. A 11, 157 (1996) [arXiv:hep-th/9509162].

[9] H. Itoyama and A. Morozov, "Integrability and Seiberg-Witten Theory: Curves and Periods," Nucl. Phys. B 477, 855 (1996) [arXiv:hep-th/9511126]. H. Itoyama and A. Morozov, "Prepotential and the Seiberg-Witten Theory," Nucl. Phys. B 491, 529 (1997) [arXiv:hep-th/9512161].

[10] E. D'Hoker and D. H. Phong, "Calogero-Moser systems in SU(N) Seiberg-Witten theory," Nucl. Phys. B 513, 405 (1998) [arXiv:hep-th/9709053].

[11] E. D'Hoker and D. H. Phong, "Spectral curves for super-Yang-Mills with adjoint hypermultiplet for general Lie algebras," Nucl. Phys. B 534, 697 (1998) [arXiv:hep-th/9804126]. "Calogero-Moser and Toda Systems for Twisted and Untwisted Affine Lie Algebras," Nucl. Phys. B 530, 611 (1998) [arXiv:hep-th/9804125]. "Calogero-Moser Lax Pairs with Spectral Parameter for General Lie Algebras," Nucl. Phys. B 530, 537 (1998) [arXiv:hep-th/9804124].

[12] E. Witten, "Solutions of four-dimensional field theories via M-theory," Nucl. Phys. B 500, 3 (1997) [arXiv:hep-th/9703166]. 
[13] S. Katz, P. Mayr and C. Vafa, "Mirror symmetry and exact solution of 4D N = 2 gauge theories. I," Adv. Theor. Math. Phys. 1, 53 (1998) [arXiv:hep-th/9706110]. S. Katz, A. Klemm and C. Vafa, "Geometric engineering of quantum field theories," Nucl. Phys. B 497, 173 (1997) [arXiv:hep-th/9609239]. S. Kachru and C. Vafa, "Exact results for $\mathrm{N}=2$ compactifications of heterotic strings," Nucl. Phys. B 450, 69 (1995) [arXiv:hep-th/9505105].

[14] A. Gorsky, "Branes and integrability in the N = 2 SUSY YM theory," Phys. Lett. B 410, 22 (1997) [arXiv:hep-th/9612238]. A. Gorsky, S. Gukov and A. Mironov, "SUSY field theories, integrable systems and their stringy/brane origin. II," Nucl. Phys. B 518, 689 (1998) [arXiv:hep-th/9710239]. S. A. Cherkis and A. Kapustin, "Singular monopoles and supersymmetric gauge theories in three dimensions," Nucl. Phys. B 525, 215 (1998) [arXiv:hep-th/9711145].

[15] N. Seiberg and E. Witten, "Gauge dynamics and compactification to three dimensions," arXiv:hep-th/9607163.

[16] S. Katz and C. Vafa, "Geometric engineering of N=1 quantum field theories," arXiv:hep-th/96011090.

[17] C. Vafa, "On N = 1 Yang-Mills in four dimensions," Adv. Theor. Math. Phys. 2, 497 (1998) [arXiv:hep-th/9801139].

[18] N. M. Davies, T. J. Hollowood, V. V. Khoze and M. P. Mattis, "Gluino condensate and magnetic monopoles in supersymmetric gluodynamics," Nucl. Phys. B 559, 123 (1999) [arXiv:hep-th/9905015].

[19] N. M. Davies, T. J. Hollowood and V. V. Khoze, "Monopoles, affine algebras and the gluino condensate," J. Math. Phys. 44, 3640 (2003) [arXiv:hep-th/0006011].

[20] N. Dorey, "An elliptic superpotential for softly broken $\mathrm{N}=4$ supersymmetric Yang-Mills theory," JHEP 9907, 021 (1999) [arXiv:hep-th/9906011].

[21] S. P. Kumar and J. Troost, "Geometric construction of elliptic integrable systems and $\mathrm{N}=1^{*}$ superpotentials," JHEP 0201, 020 (2002) [arXiv:hep-th/0112109].

[22] A. Hanany and J. Troost, "Orientifold planes, affine algebras and magnetic monopoles," JHEP 0108, 021 (2001) [arXiv:hep-th/0107153].

[23] M. Bershadsky, K. A. Intriligator, S. Kachru, D. R. Morrison, V. Sadov and C. Vafa, "Geometric singularities and enhanced gauge symmetries," Nucl. Phys. B 481, 215 (1996) [arXiv:hep-th/9605200].

[24] J. Park, "Orientifold and F-theory duals of CHL strings," Phys. Lett. B 418, 91 (1998) [arXiv:hep-th/9611119].

[25] V.I. Inozemtsev, "Lax presentation with spectral parameter on a torus for integrable particle systems," Lett. Math. Phys. 17 (1989) 11; "The finite Toda Lattices", Commun. Math. Phys. 121 (1989) 629. 
[26] A. J. Bordner and R. Sasaki, "Calogero-Moser models. III: Elliptic potentials and twisting," Prog. Theor. Phys. 101, 799 (1999) [arXiv:hep-th/9812232].

A. J. Bordner, R. Sasaki and K. Takasaki, "Calogero-Moser models. II: Symmetries and foldings," Prog. Theor. Phys. 101, 487 (1999) [arXiv:hep-th/9809068].

A. J. Bordner, E. Corrigan and R. Sasaki, "Calogero-Moser models. I: A new formulation," Prog. Theor. Phys. 100, 1107 (1998) [arXiv:hep-th/9805106].

[27] G. Veneziano and S. Yankielowicz, "An Effective Lagrangian For The Pure N=1 Supersymmetric Yang-Mills Theory," Phys. Lett. B 113, 231 (1982).

[28] K. Hori and C. Vafa, "Mirror symmetry," arXiv:hep-th/0002222.

[29] M. Aganagic, K. Intriligator, C. Vafa and N. P. Warner, "The glueball superpotential," arXiv:hep-th/0304271.

[30] R. Dijkgraaf and C. Vafa, "Matrix models, topological strings, and supersymmetric gauge theories," Nucl. Phys. B 644, 3 (2002) [arXiv:hep-th/0206255]. "On geometry and matrix models," Nucl. Phys. B 644, 21 (2002) [arXiv:hep-th/0207106]. R. Dijkgraaf and C. Vafa, "A perturbative window into non-perturbative physics," arXiv:hep-th/0208048.

R. Dijkgraaf, M. T. Grisaru, C. S. Lam, C. Vafa and D. Zanon, "Perturbative computation of glueball superpotentials," Phys. Lett. B 573, 138 (2003) [arXiv:hep-th/0211017].

[31] W. Nahm, "Selfdual Monopoles And Calorons," BONN-HE-83-16 Presented at 12th Colloq. on Group Theoretical Methods in Physics, Trieste, Italy, Sep 5-10, 1983

[32] K. M. Lee and P. Yi, Phys. Rev. D 56, 3711 (1997) [arXiv:hep-th/9702107].

[33] K. M. Lee and C. h. Lu, Phys. Rev. D 58, 025011 (1998) [arXiv:hep-th/9802108].

[34] T. C. Kraan and P. van Baal, "Exact T-duality between calorons and Taub - NUT spaces," Phys. Lett. B 428, 268 (1998) [arXiv:hep-th/9802049]. T. C. Kraan and P. van Baal, "Periodic instantons with non-trivial holonomy," Nucl. Phys. B 533, 627 (1998) [arXiv:hep-th/9805168]. T. C. Kraan and P. van Baal, "Monopole constituents inside SU(n) calorons," Phys. Lett. B 435, 389 (1998) [arXiv:hep-th/9806034].

[35] K. M. Lee, "Instantons and magnetic monopoles on $\mathrm{R}^{* *} 3 \times \mathrm{S}(1)$ with arbitrary simple gauge groups," Phys. Lett. B 426, 323 (1998) [arXiv:hep-th/9802012].

[36] A. Dabholkar, "Lectures on orientifolds and duality," arXiv:hep-th/9804208.

[37] J. H. Schwarz, "An SL(2,Z) multiplet of type IIB superstrings," Phys. Lett. B 360, 13 (1995) [Erratum-ibid. B 364, 252 (1995)] [arXiv:hep-th/9508143]. P. S. Aspinwall, "Some relationships between dualities in string theory," Nucl. Phys. Proc. Suppl. 46, 30 (1996) [arXiv:hep-th/9508154]. 
[38] A. Hanany and B. Kol, "On orientifolds, discrete torsion, branes and M theory," JHEP 0006, 013 (2000) [arXiv:hep-th/0003025].

[39] E. G. Gimon, "On the M-theory interpretation of orientifold planes," arXiv:hep-th/9806226.

[40] M. A. Olshanetsky and A. M. Perelomov, "Completely Integrable Classical Systems Connected With Semisimple Lie Algebras. 1," Lett. Math. Phys. 1, 187 (1976). "Classical Integrable Finite Dimensional Systems Related To Lie Algebras," Phys. Rept. 71 (1981) 313.

[41] H. Osborn, "Topological Charges For N=4 Supersymmetric Gauge Theories And Monopoles Of Spin 1," Phys. Lett. B 83, 321 (1979); A. Sen, "Dyon - monopole bound states, selfdual harmonic forms on the multi - monopole moduli space, and SL(2,Z) invariance in string theory," Phys. Lett. B 329, 217 (1994) [arXiv:hep-th/9402032]; J. P. Gauntlett and D. A. Lowe, "Dyons and S-Duality in N=4 Supersymmetric Gauge Theory," Nucl. Phys. B 472, 194 (1996) [arXiv:hep-th/9601085]; K. M. Lee, E. J. Weinberg and P. Yi, "Electromagnetic Duality and $S U(3)$ Monopoles," Phys. Lett. B 376, 97 (1996) [arXiv:hep-th/9601097]; K. M. Lee, E. J. Weinberg and P. Yi, "The Moduli Space of Many BPS Monopoles for Arbitrary Gauge Groups," Phys. Rev. D 54, 1633 (1996) [arXiv:hep-th/9602167]; G. W. Gibbons, "The Sen conjecture for fundamental monopoles of distinct type," Phys. Lett. B 382, 53 (1996) [arXiv:hep-th/9603176].

[42] N. Dorey, C. Fraser, T. J. Hollowood and M. A. C. Kneipp, "S-duality in N=4 supersymmetric gauge theories," Phys. Lett. B 383, 422 (1996) [arXiv:hep-th/9605069].

[43] K. M. Lee and P. Yi, Phys. Rev. D 58, 066005 (1998) [arXiv:hep-th/9804174].

[44] J. P. Gauntlett and J. A. Harvey, "S duality and the dyon spectrum in N=2 superYang-Mills theory," Nucl. Phys. B 463, 287 (1996) [arXiv:hep-th/9508156]; J. P. Gauntlett, N. Kim, J. Park and P. Yi, "Monopole dynamics and BPS dyons in N = 2 super-Yang-Mills theories," Phys. Rev. D 61, 125012 (2000) [arXiv:hep-th/9912082]; M. Stern and P. Yi, "Counting Yang-Mills dyons with index theorems," Phys. Rev. D 62, 125006 (2000) [arXiv:hep-th/0005275].

[45] R. Boels, J. de Boer, R. Duivenvoorden and J. Wijnhout, "Nonperturbative superpotentials and compactification to three dimensions," arXiv:hep-th/0304061. "Factorization of Seiberg-Witten curves and compactification to three dimensions," arXiv:hep-th/0305189.

[46] N. Nekrasov, "Five dimensional gauge theories and relativistic integrable systems," Nucl. Phys. B 531, 323 (1998) [arXiv:hep-th/9609219].

[47] H. W. Braden, A. Marshakov, A. Mironov and A. Morozov, "The Ruijsenaars-Schneider model in the context of Seiberg-Witten theory," Nucl. Phys. B 
558, 371 (1999) [arXiv:hep-th/9902205]. Y. Ohta, "Instanton correction of prepotential in Ruijsenaars model associated with $\mathrm{N}=2 \mathrm{SU}(2)$ Seiberg-Witten theory," J. Math. Phys. 41, 4541 (2000) [arXiv:hep-th/9909196]. H. W. Braden, A. Marshakov, A. Mironov and A. Morozov, "Seiberg-Witten theory for a non-trivial compactification from five to four dimensions," Phys. Lett. B 448, 195 (1999) [arXiv:hep-th/9812078]. A. M. Khvedelidze and D. M. Mladenov, "Euler-Calogero-Moser system from SU(2) Yang-Mills theory," Phys. Rev. D 62, 125016 (2000) [arXiv:hep-th/9906033]. A. Gorsky and A. Mironov, "Solutions to the reflection equation and integrable systems for $\mathrm{N}=2$ SQCD with classical groups," Nucl. Phys. B 550, 513 (1999) [arXiv:hep-th/9902030]. I. M. Krichever, "Elliptic analog of the Toda lattice," arXiv:hep-th/9909224. K. Takasaki, "Elliptic Calogero-Moser systems and isomonodromic deformations," math.qa/9905101.

[48] S. N. M. Ruijsenaars and H. Schneider, "A New Class Of Integrable Systems And Its Relation To Solitons," Annals Phys. 170 (1986) 370. 